\title{
Steady-state Fano coherences in a V-type system driven by polarized incoherent light
}

\author{
Suyesh Koyu, ${ }^{1}$ Amro Dodin $\odot,{ }^{2}$ Paul Brumer $\odot,{ }^{3}$ and Timur V. Tscherbul $\odot^{1, *}$ \\ ${ }^{1}$ Department of Physics, University of Nevada, Reno, Nevada 89557, USA \\ ${ }^{2}$ Department of Chemistry, MIT, Cambridge, Massachusetts 02139, USA \\ ${ }^{3}$ Chemical Physics Theory Group, Department of Chemistry, and Center for Quantum Information and Quantum Control, \\ University of Toronto, Toronto, Ontario, M5S 3H6, Canada
}

(Received 24 January 2020; accepted 16 February 2021; published 31 March 2021)

\begin{abstract}
We explore the properties of steady-state noise-induced (Fano) coherences generated in a three-level V-system continuously pumped by polarized incoherent light in the absence of coherent driving. By solving the nonsecular Bloch-Redfield quantum master equation, we obtain the ratio of the stationary coherences to excited-state populations, $\mathcal{C}=\left(1+\frac{\Delta^{2}}{\gamma(r+\gamma)}\right)^{-1}$, which quantifies the impact of steady-state coherences on excited-state dynamical observables of the V-system. The ratio is maximized when the excited-state splitting $\Delta$ is small compared to either the spontaneous decay rate $\gamma$ or the incoherent pumping rate $r$. We demonstrate that while the detrimental effects of a strongly decohering environment generally suppress the coherence-to-population ratio by the factor $\simeq \gamma_{d} / \gamma$, an intriguing regime exists where the $\mathcal{C}$ ratio displays a maximum as a function of the dephasing rate $\gamma_{d}$. We attribute the surprising dephasing-induced enhancement of stationary Fano coherences to the environmental suppression of destructive interference of individual incoherent excitations generated at different times. We clarify the physical basis for the steady-state Fano coherence, whose imaginary part is identified with the nonequilibrium flux across a pair of qubits coupled to two independent thermal baths or, equivalently, the spontaneous emission flux from the right qubit to the right bath, unraveling a direct connection between the seemingly unrelated phenomena of incoherent driving of multilevel quantum systems and nonequilibrium quantum transport in qubit networks. We further establish the equivalence between the two-qubit system and a V-system, each of whose transitions is driven simultaneously by both baths. The real part of the steady-state Fano coherence is found to be proportional to the deviation of excited-state populations from their values in thermodynamic equilibrium, making it possible to observe signatures of steady-state Fano coherences in excited-state populations. Finally, we put forward an experimental proposal for observing steady-state Fano coherences by detecting the total fluorescence signal emitted by Calcium atoms excited by polarized versus isotropic incoherent light. Our analysis paves the way toward further theoretical and experimental studies of nonequilibrium coherent steady states in thermally driven atomic and molecular systems and for the exploration of their potential role in quantum thermodynamics and in biological processes.
\end{abstract}

DOI: 10.1103/PhysRevResearch.3.013295

\section{INTRODUCTION}

Quantum coherence is widely regarded as an essential resource [1] for quantum information processing [2], quantum sensing [3], and quantum interferometry [4]. The inevitable interaction of quantum systems with an external environment is generally believed to lead to the decay of quantum coherence in a process known as decoherence [5]. As decoherence upsets the unitary time evolution necessary for the successful operation of quantum bits and sensors, understanding and controlling decoherence mechanisms is a key research goal of quantum science and technology.

\footnotetext{
*ttscherbul@unr.edu

Published by the American Physical Society under the terms of the Creative Commons Attribution 4.0 International license. Further distribution of this work must maintain attribution to the author(s) and the published article's title, journal citation, and DOI.
}

An additional motivation to study decoherence comes from recent experimental and theoretical studies of quantum effects in biological processes, such as photosynthetic energy transfer [6-8], vision [9,10], and avian magnetoreception [11]. These studies have indicated that nontrivial quantum effects such as coherence and entanglement [6,7] can persist under noisy conditions typical of biological environments at room temperature. In all of the experimental studies performed to date, quantum coherence was introduced into the system by means of coherent (ultrashort) laser pulses [6-8]. However, biologically relevant photosynthetic light-harvesting is driven by natural sunlight, which is an incoherent mixture of number states, raising an important question: can excitation by incoherent light alone (i.e., in the absence of coherent laser driving) generate coherence in multilevel quantum systems [8,12-18]

Surprisingly, the answer to this question is "yes," although the coherences are distinctly different from coherent light-induced effects [8-10]. Incoherent radiative transitions between manifolds of closely spaced energy levels can 
(a)

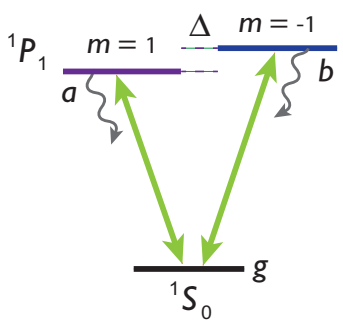

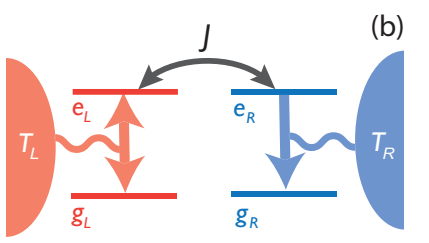

(c)

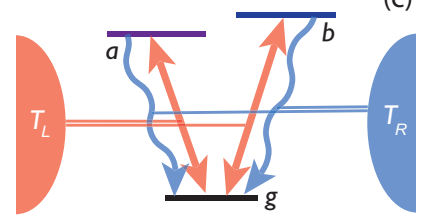

FIG. 1. (a) Energy level diagram of the V-system composed of a single ground state $g$ and two nearly degenerate excited levels $a$ and $b$. In our proposed experimental realization of the V-system, the ground state corresponds to the ${ }^{1} \mathrm{~S}_{0}$ level of atomic $\mathrm{Ca}$ and the excited states corresponds to the ${ }^{1} \mathrm{~S}_{1}$ levels of atomic $\mathrm{Ca}$ with the total electron orbital angular momentum $J=1$ and its projection $m_{J}= \pm 1$ on the magnetic field axis. The excited-state energy splitting $\Delta$ is continuously tunable with an external magnetic field. The rates of spontaneous decay (wavy arrows) are given by $\gamma_{i}(i=a, b)$, and those of incoherent pumping (straight arrows) by $r_{i}=\gamma_{i} \bar{n}$. (b) An alternative representation of the V-system driven by polarized incoherent light as a pair of coherently coupled qubits interacting with two independent thermal baths at different temperatures. (c) An equivalent representation of the two-qubit model as a V-system, in which the transitions $g \leftrightarrow a$ and $g \leftrightarrow b$ are driven simultaneously by the left and right baths (see text for details).

interfere to produce noise-induced Fano coherences in multilevel atomic and molecular systems even in the absence of coherent (e.g., laser) driving [13,16-26]. The interference manifests itself through the cross terms in the quantum optical (Bloch-Redfield) master equation, which correspond to the interaction of a common incoherent light mode with the dipole moments of the transitions from the same initial state to different final states $[13,17,18,25,26]$.

Initial theoretical studies of Fano coherences highlighted their significance in the context of lasing without inversion [23] and quantum jumps in trapped ions [24]. Agarwal and Menon [27] explored the conditions under which the three-level V-system (see Fig. 1) pumped by incoherent light approaches thermodynamic equilibrium in the long-time limit. They showed that in the absence of symmetry between incoherent pumping and spontaneous decay, nontrivial steadystate coherences can arise, leading to nonequilibrium steady states. Kozlov, Rostovtsev, and Scully showed that incoherent pumping of the $\mathrm{V}$-system with degenerate upper levels can create coherent population-locked states, which depend on the initial state of the system [25]. Recent theoretical work by Dorfman, Scully, Mukamel, and co-workers [20,21] reinvigorated interest in noise-induced Fano coherences by showing that they can enhance the efficiency of quantum heat engines by breaking the principle of detailed balance. Note, however, that a problem has recently been discovered [28] with the master equation approach used in Refs. [20,21] which gives negative population dynamics at long times.

We have explored the dynamical properties of Fano coherences in realistic $\mathrm{V}$-systems with nondegenerate excited states, establishing the existence of two dynamical regimes depending on the ratio of the excited-state splitting $\Delta$ to the radiative decay rate $\gamma[13,17,26]$. In the underdamped regime characterized by $\Delta / \gamma \gg 1$, Fano coherence exhibit damped oscillations that decay on the timescale $\tau \simeq 1 / \gamma[13,17]$. In the overdamped regime, where the excited-state splitting is smaller than the radiative decay rate, we observe long-lived quasisteady-state coherences with the lifetime $\tau=\frac{2}{\gamma}\left(\frac{\Delta}{\gamma}\right)^{-2}$ $[13,17]$. We have also explored the dynamics of the V-system driven by polarized incoherent light [29] and proposed an experimental scheme for observing noise-induced coherence dynamics in calcium atoms driven by incoherent radiation.

The majority of theoretical studies of noise-induced Fano coherences have focused on the dynamics induced by isotropic incoherent light, whose polarization $\left(\boldsymbol{\epsilon}_{\lambda}\right)$ and propagation (k) vectors point randomly in all directions. The coherence properties of isotropic incoherent light [such as its first-order degree of coherence $g^{(1)}(\tau)$ ] are independent of $\boldsymbol{\epsilon}_{\lambda}$ and $\mathbf{k}$ [30]. As a consequence, the light-matter interaction only depends on the angles between the dipole moment vectors $\boldsymbol{\mu}_{i j}$ and $\boldsymbol{\mu}_{k l}$ for radiative transitions between the system eigenstates $|i\rangle \leftrightarrow|j\rangle$ and $|k\rangle \leftrightarrow|l\rangle[16,31,32]$. The resulting quantum master equation treats incoherent excitation and decay dynamics on an equal footing, leading to the decay of quantum coherences in the long-time limit and the establishment of the canonical equilibrium steady state [27], although the time it takes to reach this state can be extremely long for nearly-degenerate levels $[13,17,26]$.

In contrast, when a quantum system is excited by polarized incoherent light, only one polarization mode of the light interacts with the transition dipole moment vectors [10,29], whereas spontaneous emission still occurs in all directions (in the absence of an external cavity). As a result of the imbalance between incoherent pumping and spontaneous emission, the excitation with polarized incoherent light can lead to the emergence of nonequilibrium steady states (NESS) featuring coherences in the energy eigenstate basis and populations deviating significantly from their expected canonical values [27]. By coherent NESS we mean a nonequilibrium steady state with a system density matrix that has off-diagonal elements in the system energy eigenstate basis. These steady states are distinct from the more commonly occurring NESS that arise due to the additional selection rules for excitation with polarized light. For instance, the $\Delta m_{J}= \pm 1$ electric dipole selection rule implies that the $\left|J=1, m_{J}=0\right\rangle$ excited state cannot be populated from the ground $\left|J=0, m_{J}=0\right\rangle$ state irradiated by incoherent light polarized in the $x$ direction [29]. While important for optical pumping, these NESS manifest themselves through the appearance of dark states, and do not feature coherences in the energy eigenstate basis of the system, so we will not consider them further in this work.

In contrast to the case of excitation with isotropic incoherent light, very little attention has been devoted to the NESS and steady-state Fano coherences induced by polarized incoherent excitation of multilevel quantum systems. Agarwal and Menon derived analytical expressions for steady-state Fano coherences in a three-level V-system driven by broadband incoherent radiation [27]. We have shown that the steady-state 
coherences can occur in Calcium atoms driven by polarized incoherent light [29]. However, even the most basic properties of steady-state Fano coherences (such as their dependence of the system and excitation parameters, and their sensitivity to decoherence) remain unexplored, despite widespread occurrence of polarized sunlight in nature $[33,34]$, and the resultant possibility of noise-induced coherences playing a role in biological excitation processes.

Here, we present a theoretical analysis of steady-state Fano coherences in a three-level V-system driven by polarized incoherent light in the absence of coherent laser-induced excitation. The V-system serves as a minimal model, in which to study the quantum dynamics of noise-induced coherence generation [13,15] and energy transfer [15,35-38]. We obtain analytic results for the steady-state coherences and explore their dependence on the system parameters. Our results suggest that the coherences are maximized when the excited-state splitting is small compared to the radiative decay rate (i.e., in the large-molecule limit [13]). We clarify the physical origin of the coherences by establishing an equivalence between the quantum master equation describing the V-system excited by polarized incoherent radiation and that describing the dynamics of nonequilibrium energy transport across a pair of quantum two-level systems (qubits) coupled to two independent thermal baths [39]. Finally, we present an experimental scenario for observing the signatures of steady-state Fano coherences in the fluorescence signal emitted by calcium atoms excited by polarized incoherent light. Our analysis paves the way toward further theoretical and experimental studies of NESS and steady-state Fano coherences in thermally driven atomic and molecular systems, and for the exploration of their potential role in natural processes induced and sustained by solar light.

In the rest of this paper, we will present the details of our theoretical calculations based on the nonsecular BlochRedfield (BR) quantum master equation (Sec. II). In Sec. III, we report the results for steady-state Fano coherences as a function of the system and excitation parameters. In Sec. III A, we establish the physical basis for steady-state Fano coherences using a two-bath transport model. The effects of relaxation and decoherence are examined in Sec. III B. An experimental proposal for observing Fano coherences in calcium atoms in put forward in Sec. IV. Section V summarizes and discusses the main results of this work.

\section{THEORY AND METHODS: ANALYTICAL SOLUTIONS TO BLOCH-REDFIELD EQUATIONS IN THE STEADY STATE}

To model the quantum dynamics of the V-system driven by polarized incoherent radiation, we use the Bloch-Redfield (BR) quantum master equation for the system's density matrix $[13,17,18,27,40]$, which can be derived from the Liouvillevon Neumann equation for the density operator of the system + bath complex [40,41], where the role of the bath is played by the incoherent radiation. The derivation assumes that (1) the system-bath interaction is weak compared to the system energy scales (Born approximation) so that the system-bath density matrix can be factorized into the system and bath parts, and (2) the surrounding bath fluctuations are much faster than the system's evolution (Markov approximation). Importantly, we do not assume the validity of the secular approximation, which neglects the elements of the relaxation (Redfield) tensor responsible for the coupling between populations and coherences $[13,40]$. This approximation is only justified in the absence of Liouvillian degeneracies [42] in the energy level spectrum of the system, which is clearly not the case for the V-system shown in Fig. 1(a), where levels $a$ and $b$ are nearly degenerate. Unlike in the optical Bloch equations, which describe a quantum system driven by a coherent laser source, the coupling between the populations and coherences comes from the nonsecular terms in the BR equation, which are due to the interference of two incoherent transition pathways $g \leftrightarrow a$ and $g \leftrightarrow b$. Note that in the absence of the population-to-coherence coupling, the Bloch-Redfield equations reduce to the standard Pauli rate equations commonly used to describe thermal driving [13,20,27].

Expressed in the system eigenstate basis, the BR equations take the form $[13,17,18,27]$

$$
\begin{aligned}
\dot{\rho}_{i i}= & -\left(r_{i}+\gamma_{i}+\Gamma_{i}\right) \rho_{i i}+r_{i} \rho_{g g}-\sqrt{r_{a} r_{b}} \rho_{a b}^{R}, \\
\dot{\rho}_{a b}= & -\frac{1}{2}\left(r_{a}+r_{b}+\gamma_{a}+\gamma_{b}+2 \gamma_{d}\right) \rho_{a b} \\
& -i \Delta \rho_{a b}+\sqrt{r_{a} r_{b}} \rho_{g g}-\frac{1}{2} \sqrt{r_{a} r_{b}}\left(\rho_{a a}+\rho_{b b}\right),
\end{aligned}
$$

where $\rho_{a b}=\rho_{a b}^{R}+i \rho_{a b}^{I}$ is the coherence between the excited eigenstates $|a\rangle$ and $|b\rangle$ [see Fig. 1(a)], $\gamma_{i}$ is the spontaneous decay rate of the $i$-th excited-state level, $r_{i}=\bar{n} \gamma_{i}$ is the incoherent pumping rate, and $\bar{n}=\left[e^{\hbar \omega_{a g} / k_{B} T}-1\right]^{-1}$ is the average photon occupation number of the incoherent radiation field at the temperature $T$, which we assume to be polarized in the $x$ direction of the space-fixed coordinate frame [29]. Equations (1) correspond to case I ( $a=1, b=0)$ defined by Agarwal and Menon [27], where interference is present only in absorption (due to the anisotropy of the $x$-polarized incoherent light) but not in spontaneous emission, since we consider the V-system with orthogonal transition dipole moments (such as the $\mathrm{Ca}$ atom in Sec. IV).

We further note that Eq. (1) neglects the one-photon coherences between the ground and each of the excited levels of the $\mathrm{V}$-system. This is justified because the splitting between these levels $\left(\simeq 10^{15} \mathrm{~Hz}\right)$ is much larger than the inverse timescale of interest for the system evolution $\left(10^{-10}-10^{-6} \mathrm{~s}\right)$. Thus, on this timescale, the one-photon coherences oscillate much more rapidly than their two-photon counterparts, and their time evolutions decouple under the partial secular approximation [16].

The environmental relaxation and decoherence channels (to be considered in Sec. III B) are described by the phenomenological rates $\Gamma_{i}$ and $\gamma_{d}$. Note that Eq. (1) assumes that nonradiative coupling with an external environment (e.g., phonons) cannot generate coherence between the eigenstates of the V-system [43], i.e., that Fano coherence can only be generated by the light-matter interaction through the $\sqrt{r_{a} r_{b}} \rho_{g g}$ term in Eq. (1). Isotropic incoherent excitation in the presence of coherence-generating system-phonon interactions have been considered elsewhere $[10,16,19,36]$.

We will consider the case of a symmetric V-system with $\gamma_{a}=\gamma_{b}=\gamma, r_{a}=r_{b}=r$, and $\Gamma_{a}=\Gamma_{b}=\Gamma$ which contains all of the essential physics while drastically simplifying the 
analytical solution of the BR equations (1). The symmetry implies that $\rho_{a a}(t)=\rho_{b b}(t)$ at all times $[13,26]$, a consequence of the symmetric initial condition $\rho_{g g}(0)=1$. Note that the relation $\rho_{a a}=\rho_{b b}$ will hold in the steady state irrespective of the initial conditions because the solution of Eq. (5) is uniquely defined for $\Delta>0$ (see below).

Combining this with the requirement that the density matrix must have unit trace $\left[\rho_{a a}(t)+\rho_{b b}(t)+\rho_{g g}(t)=1\right]$, we get $2 \rho_{a a}(t)+\rho_{g g}(t)=1$, which allows us to eliminate $\rho_{b b}$ and $\rho_{g g}$ from Eqs. (1). The resulting BR equations for the symmetric V-system may be written as

$$
\begin{aligned}
& \dot{\rho}_{a a}=-(3 r+\gamma+\Gamma) \rho_{a a}-r \rho_{a b}^{R}+r, \\
& \dot{\rho}_{a b}^{R}=-3 r \rho_{a a}-\left(r+\gamma+\gamma_{d}\right) \rho_{a b}^{R}+\Delta \rho_{a b}^{I}+r, \\
& \dot{\rho}_{a b}^{I}=-\Delta \rho_{a b}^{R}-\left(r+\gamma+\gamma_{d}\right) \rho_{a b}^{I} .
\end{aligned}
$$

Here, as above, the phenomenological parameters $\Gamma$ and $\gamma_{d}$ account for the effects of relaxation and decoherence between the excited states of the V-system due to, e.g., nonradiative processes. These effects will be explored in Sec. III B below. In Sec. III, we will begin by considering only the radiative processes, setting $\Gamma=\gamma_{d}=0$.

The BR quantum master equations (2) can be rewritten in matrix-vector notation as [17]

$$
\dot{\mathbf{x}}(t)=\mathbf{A x}(t)+\mathbf{d},
$$

where $\mathbf{x}(t)=\left[\rho_{a a}(t), \rho_{a b}^{R}(t), \rho_{a b}^{I}(t)\right]^{T}$ is the state vector in the Liouville representation [17], $\mathbf{d}=[r, r, 0]^{T}$ is the driving vector corresponding to the V-system being initially in the ground state $\left[\rho_{g g}(t=0)=1\right]$, and the coefficient matrix $\mathbf{A}$ is given by

$$
\mathbf{A}=\left[\begin{array}{ccc}
-(3 r+\gamma) & -r & 0 \\
-3 r & -(r+\gamma) & \Delta \\
0 & -\Delta & -(r+\gamma)
\end{array}\right]
$$

In this work, we are primarily interested in the steady-state behavior of the V-system irradiated by incoherent light. Setting $\dot{\mathbf{x}}(t)=0$ in Eq. (3) we obtain the steady-state solution

$$
\mathbf{x}_{s}=-\mathbf{A}^{-1} \mathbf{d} .
$$

The steady-state solution is unique only if the coefficient matrix $\mathbf{A}$ is nonsingular, i.e., $\operatorname{det}(\mathbf{A})=3 r^{2}(r+\gamma)-(3 r+$ $\gamma)\left[\Delta^{2}+(r+\gamma)^{2}\right] \neq 0$. The determinant is nonzero in general but it tends to zero as $\Delta \rightarrow 0$ and $r / \gamma \rightarrow \infty$, leading to initial condition-dependent population-locked steady states similar to those explored in Ref. [25]. However, due to, e.g., differential level shifts experienced by the excited levels interacting with the pumping field, most real-world V-systems will have $\Delta>0$. As a result, $\operatorname{det}(\mathbf{A}) \neq 0$ and the steady-state solution (5) is uniquely defined. We note that the steadystate solution (5) is, by definition, not sensitive to short-time transient dynamics. Therefore, unlike the transient Fano coherences explored in our previous work $[13,17,18,26]$, the steady-state coherences are independent of how rapidly the system-radiation interaction is turned on [18].

\section{STEADY-STATE FANO COHERENCES: RESULTS AND DISCUSSION}

Substituting the inverse of matrix A into Eq. (5), we find analytical expressions for excited-state populations and the real part of Fano coherence in the steady state [44]

$$
\begin{aligned}
& \rho_{a a}=\frac{r\left[\Delta^{2}+(r+\gamma)^{2}-r(r+\gamma)\right]}{(3 r+\gamma)\left[\Delta^{2}+(r+\gamma)^{2}\right]-3 r^{2}(r+\gamma)}, \\
& \rho_{a b}^{R}=\frac{r \gamma(r+\gamma)}{(3 r+\gamma)\left[\Delta^{2}+(r+\gamma)^{2}\right]-3 r^{2}(r+\gamma)}
\end{aligned}
$$

with the imaginary part of the coherence $\rho_{a b}^{I}=-\frac{\Delta}{(r+\gamma)} \rho_{a b}^{R}$, in agreement with the results of Ref. [27]. Below we analyze the remarkable properties of steady-state Fano coherences not explored in Ref. [27], and study their dependence on the excited-state splitting $\Delta / \gamma$ and the incoherent pumping rate $r=\bar{n} \gamma$. The effects of relaxation and decoherence are considered in the following section.

First, we observe that neither the real nor imaginary part of the Fano coherence vanishes in the steady state, in contrast to the previously explored cases of incoherent driving with isotropic (unpolarized) light $[13,17,26]$. Figure 2 shows the time evolution of excited-state populations and coherences in the V-system driven by polarized incoherent light. The results are obtained by numerical integration of Eq. (2). The equilibration timescale depends on the dynamical regimes of the V-system classified in our previous work [29,44], which are determined by the value of excited-state splitting $\Delta / \gamma$ and the incoherent pumping rate $r=\bar{n} \gamma$. Regardless of the transient dynamics, the V-system eventually reaches a steady state characterized by nonzero values of Fano coherences shown by the dashed lines in Fig. 2, which are in excellent agreement with the analytical expressions (6).

It may seem surprising that incoherent driving excites a coherent superposition rather than an incoherent mixture of energy eigenstates. The presence of coherences seems incongruent with an incoherent nature of the light source, which is thought of as having an entirely random phase at every instant. For such a source excitations generated at different times will pick up a different phase from the fluctuating light field, yielding a random phase that vanishes upon averaging over the realizations of the incoherent source. However, this phase averaging argument does not hold for excitations generated at the same instant, provided the corresponding transition dipole moment vectors are nonorthogonal $[13,16,17]$. In this case, both excitations will accrue the same (randomly selected) phase from the incoherent drive, resulting in an in-phase superposition of the two eigenstates. That is, while the relative phase between excitations generated at different times is uncorrelated, the relative phase of simultaneous excitations is deterministic since both arise by interacting with the same state of the light field [10].

To further understand the counterintuitive emergence of the coherent steady-state, we consider the dynamics of the contributing coherent excitations. As noted earlier in this section, an incoherent light source generates a coherent drive due to the presence of simultaneous excitations from the ground state to two different excited states. This process generates an in-phase superposition of two energy eigenstates. Once this 

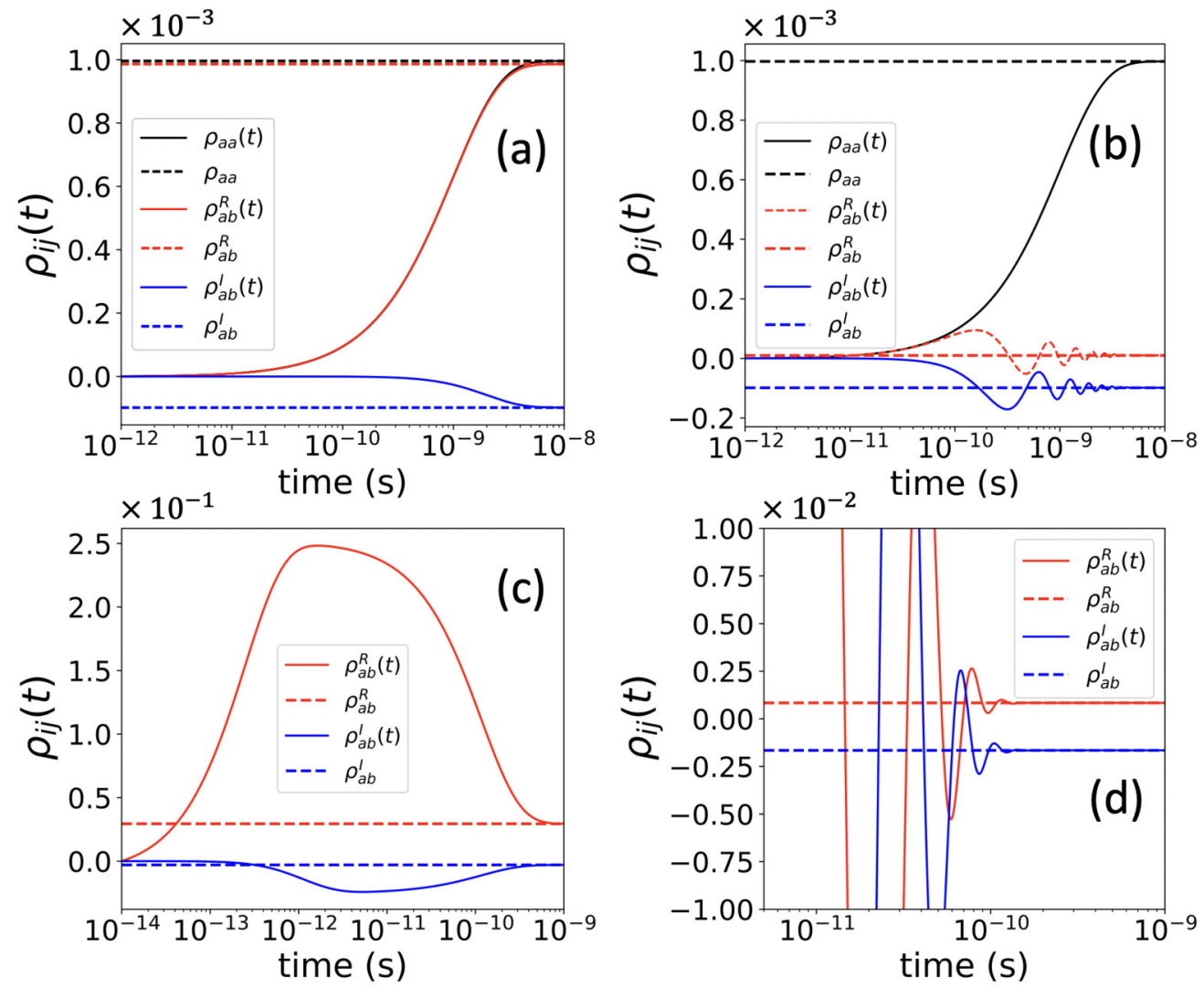

FIG. 2. Time evolution of excited-state populations and Fano coherences in the V-system weakly [(a) and (b)] and strongly [(c) and (d)] driven by $x$-polarized incoherent light. (a) Overdamped regime with $\bar{n}=10^{-3}$ and $\Delta / \gamma=10^{-1}$. (b) Underdamped regime with $\bar{n}=10^{-3}$ and $\Delta / \gamma=10$; (c) $\bar{n}=10^{3}$ and $\Delta / \gamma=10^{2}$, and (d) $\bar{n}=10^{2}$ and $\Delta / \gamma=2 \times 10^{2}$. The steady-state values of Fano coherences are shown by horizontal dashed lines. Note that the excited-level populations shown in (a) and (b) grow monotonically, whereas the real and imaginary Fano coherences in (b) and (d) display underdamped coherent oscillations.

excitation is generated, it undergoes unitary evolution, accruing a periodic relative phase with frequency $\Delta$. Moreover, it will slowly dephase with rate $\gamma_{d}$ and relax to the ground state with rate $\gamma+r+\Gamma$ [see Eq. (1)]. The timescales and effects of these contributions on the dynamics of a single excitation are shown schematically in Fig. 3(a).

In incoherent driving, the source is assumed to be continuously acting on the system, generating new excitations at every instant in time. At each instant, therefore, a new in- phase superposition is generated and undergoes the dynamics described above. The resulting ensemble dynamics are then obtained by summing over the contributions of all of these different excitations, as shown in Fig. 3(b). If the system frequency $\Delta$ is much larger than the excitation decay rate $(\sim \gamma)$, then excitations generated at earlier times will have accrued some phase due to unitary evolution in the excited state manifold while new excitations will always produce an in-phase superposition. Summing over these contributions
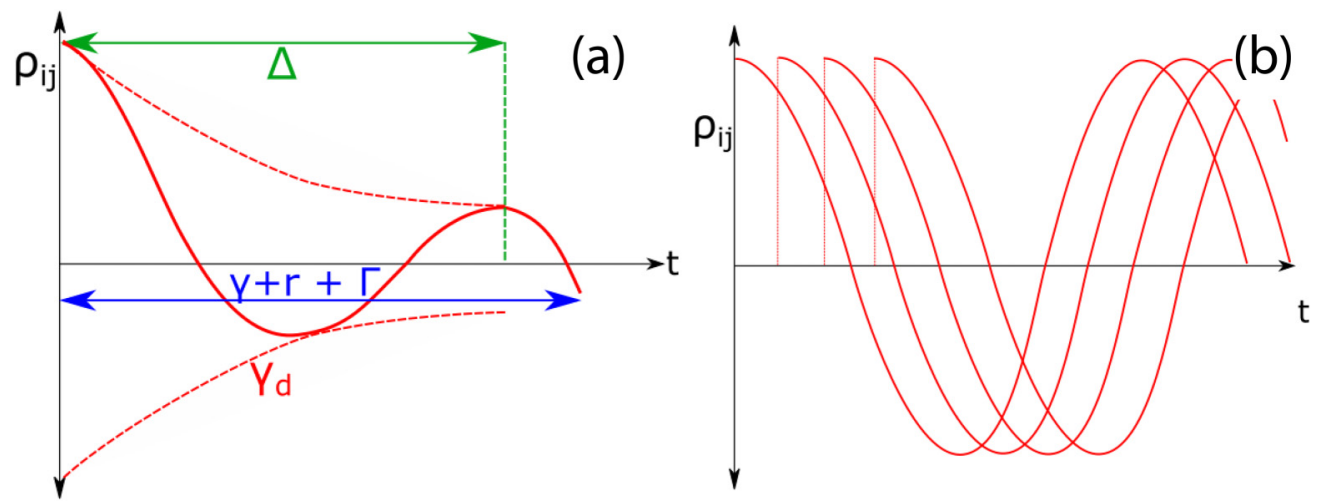

FIG. 3. Schematic depiction of the unitary time evolution and decay of a single incoherent excitation (a) and of an ensemble of incoherent excitations (b). The incoherent excitations in (b) are assumed undamped for simplicity. 
then leads to an integral over different phases, producing an ensemble dephasing with excitations generated at different times carrying different phase that eventually averages to near zero. However, when the frequency is much slower than the excitation lifetime, nearly all excitations will decay before accruing an appreciable phase. As a result, nearly all excitations will be in the initially prepared in-phase superposition, leading to the previously reported quasi-stationary coherences $[13,17]$. Generally, the interplay of these different processes need not result in an incoherent steady state, as new coherent superposition may be refreshed quickly enough to maintain coherence in the long-time limit.

In the case of unpolarized excitation that obeys the fluctuation-dissipation constraint $r=\bar{n} \gamma$, the rate of generating new excitations $r$ and their decay rate $\sim \gamma$ are directly related. This bound ensures that the delicate interplay of generating new excitations, their coherent evolution and subsequent decay are balanced to yield an incoherent thermal steady-state. However, if these rates are allowed to vary independently, it becomes possible to generate residual coherences as the balance between these different processes is broken. As discussed in the framework of the thermal transport model in Sec. III A below, polarized driving breaks this constraint by involving field modes at different temperatures, the high temperature $x$-polarization and the low-temperature isotropic vacuum. In this case, the rates are no longer balanced resulting in a coherent NESS.

The second important observation apparent from Eq. (6) and Fig. 2 is that the values of excited-state populations in the steady state deviate from those expected in canonical thermodynamic equilibrium

$$
\rho_{a a}^{(\mathrm{eq})}=\frac{r}{(3 r+\gamma)}=\frac{\bar{n}}{3 \bar{n}+1},
$$

since $r=\bar{n} \gamma$. The deviation of the population of the excited state $|a\rangle$ from canonical equilibrium then follows from Eq. (6)

$$
\begin{aligned}
\rho_{a b}^{R} & =\frac{\rho_{a a}^{(\mathrm{eq})}-\rho_{a a}}{\rho_{a a}^{(\mathrm{eq})}}, \\
\rho_{a b}^{I} & =-\frac{\Delta}{(r+\gamma)} \rho_{a b}^{R}
\end{aligned}
$$

These expressions are a central result of this work. They establish that polarized incoherent driving leads to the formation of a coherent NESS characterized by substantial Fano coherences in the energy eigenstate basis of the $\mathrm{V}$-system.

Significantly, Eq. (8) suggests that the relative deviation of excited-state populations in the NESS from their values in canonical thermodynamic equilibrium (7) is equal to the real part of the Fano coherence. In other words, the real part of the steady-state coherence is directly proportional to the difference in the populations of the excited state $|a\rangle$ with and without Fano coherence.

Equation (8) thus provides a direct connection between steady-state Fano coherences and excited-state populations of the V-system continuously driven by a polarized incoherent radiation field. As such, Eq. (8) enables direct experimental detection of the steady-state Fano coherences through measuring the deviation of excited-state populations from their canonical equilibrium values (7), as shown in Sec. IV.
Because it is typically much easier to detect eigenstate populations than coherences, steady-state Fano coherences may be significantly easier to observe experimentally than their transient counterparts [29].

The physical origin of the nonequilibrium coherences (to be further clarified in Sec. III A below) can be traced back to Fano interference between the electric dipole transitions induced by the interaction with a common incoherent light field mode. This interference is manifested in the populationto-coherence coupling terms in the BR equation (2), which show that the presence of a positive real part of the coherence $\rho_{a b}^{R}(t)$, suppresses the excited state populations.

We now turn to the study of the dependence of steady-state Fano coherences on the dimensionless parameters $\bar{n}$ and $\Delta / \gamma$ that control the dynamics of the incoherently driven V-system in the absence of relaxation and decoherence. Figure 4(a) shows the dependence of steady-state Fano coherences on the average occupation number of thermal photons $\bar{n}=r / \gamma$ in the pumping field. The coherences increase gradually with the pumping intensity and approach a common limit that does not depend on $\Delta / \gamma$. As shown in Fig. 4(b) the steady-state coherence are nearly independent of the excited-state splitting $\Delta / \gamma$ in the weak pumping limit $\bar{n} \ll 1$. At higher pumping intensities, the coherences begin to decline monotonically with increasing $\Delta / \gamma$.

To determine whether the real part of steady-state Fano coherence can reach a maximum as a function of $\Delta / \gamma$ and $\bar{n}$, we calculate the first derivatives of Eq. (9) with respect to these parameters

$$
\begin{aligned}
\frac{\partial}{\partial \bar{n}} \rho_{a b}^{R} & =\frac{\left(3 \bar{n}^{2}+2 \bar{n}+1\right)(\Delta / \gamma)^{2}+\left(\bar{n}^{2}+2 \bar{n}+1\right)}{\left[(3 \bar{n}+1)(\Delta / \gamma)^{2}+\left(4 \bar{n}^{2}+5 \bar{n}+1\right)\right]^{2}}, \\
\frac{\partial}{\partial(\Delta / \gamma)} \rho_{a b}^{R} & =-\frac{2 \bar{n}(\bar{n}+1)(\Delta / \gamma)}{\left[(3 \bar{n}+1)(\Delta / \gamma)^{2}+\left(4 \bar{n}^{2}+5 \bar{n}+1\right)\right]^{2}} .
\end{aligned}
$$

The derivatives do not vanish for $\bar{n}>0$ and $\Delta / \gamma>0$, and therefore steady-state Fano coherences do not display maxima or minima. This behavior is illustrated in Fig. 4(c), which plots the real part of steady-state Fano coherence as a function of $\bar{n}$ and $\Delta / \gamma$. The magnitude of the steady-state Fano coherence is therefore maximal at a boundary of the two-dimensional region depicted in Fig. 4(c). More specifically, the maximum is achieved in the small excited-state splitting regime $\Delta / \gamma \ll$ 1 regardless of the value of $\bar{n}$. As the pumping rate increases, the region of maximum coherence is shifted to higher values of $\Delta / \gamma$.

As an alternative coherence measure, consider the ratio $[13,17]$

$$
\mathcal{C}=\frac{\rho_{a b}^{R}}{\rho_{a a}}
$$

which quantifies the relative magnitude of coherences versus excited-state populations. A substantial value of $\mathcal{C} \simeq 1$ indicates the possibility of a significant coherent contribution to excited-state dynamics, affecting the values of observables such as fluorescence emission intensities (see Sec. IV below). 

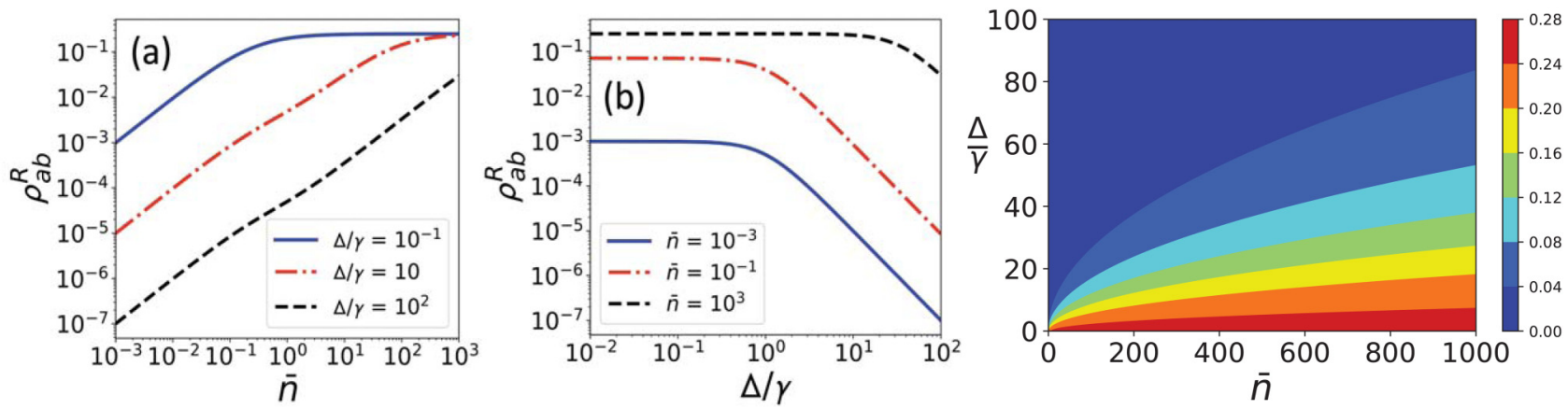

FIG. 4. (a) Steady-state Fano coherence plotted as a function of the average number of thermal photons $\bar{n}$ at fixed values of the excited-state splitting $\Delta / \gamma$. (b) Same as in panel (a) plotted vs $\Delta / \gamma$ at fixed values of $\bar{n}$ corresponding to the weak, intermediate, and strong pumping regimes. (c) Two-dimensional contour plot of steady-state Fano coherence as a function of $\Delta / \gamma$ and $\bar{n}$.

From Eq. (6), we obtain

$$
\mathcal{C}=\frac{1}{1+\frac{\Delta^{2}}{\gamma(r+\gamma)}}
$$

which is less than unity as expected from the Cauchy-Swartz inequality, $\left|\rho_{a b}\right|^{2} \leqslant \rho_{a a} \rho_{b b}$. The ratio (12) tends to unity for $\frac{\Delta^{2}}{\gamma(r+\gamma)} \ll 1$, i.e., in the regime of small excited-state splitting $\Delta$ compared to $\sqrt{\gamma(r+\gamma)}$. In the weak-pumping limit $(r \ll$ $\gamma)$, this condition simplifies to $\Delta \ll \gamma$. In the strong-pumping limit $(r \gg \gamma)$, steady-state Fano coherences are maximized relative to excited-state populations when $\Delta \ll \sqrt{r \gamma}$, which is a less restrictive condition than in the weak-pumping limit.

\section{A. Physical origin of steady-state Fano coherence: the two-bath nonequilibrium transport model}

Previous theoretical work has shown that Fano coherences induced in the V-system irradiated by isotropic incoherent radiation are transient and eventually decay back to zero $[16,17,27]$. In contrast, when the V-system is driven by polarized incoherent radiation, Fano coherences remain nonzero in the steady state, suggesting the presence of a dynamic equilibrium $[27,29]$. In our previous work, we suggested an analogy between the latter situation and one of the V-system interacting with two independent thermal baths [29], resulting in nonequilibrium transport of radiative energy across the V-system and steady-state coherences associated with it $[43,45,46]$. This kind of coherence can significantly affect the energy transfer pathways through flux networks in photosynthetic light-harvesting complexes $[38,47]$. Here, we make this analogy more precise by showing that the BR equations of motion for the V-system driven by polarized incoherent radiation are equivalent to those describing quantum transport in a system consisting of two coupled two-level systems (qubits). We identify the imaginary part of steady-state Fano coherence with the nonequilibrium flux across the dimer system. This result unravels a direct connection between the seemingly unrelated phenomena of incoherent driving of multilevel quantum systems and nonequilibrium transport.

Consider a system of two qubits coherently coupled by an exchange or dipolar interaction quantified by the hopping parameter $J$ as shown in Fig. 1(b). In the weak-pumping limit only the ground $|g\rangle=\left|g_{L} g_{R}\right\rangle$ and singly excited eigenstates

$$
\begin{aligned}
& |a\rangle=\cos \frac{\theta}{2}\left|e_{L} g_{R}\right\rangle+\sin \frac{\theta}{2}\left|g_{L} e_{R}\right\rangle, \\
& |b\rangle=-\sin \frac{\theta}{2}\left|e_{L} g_{R}\right\rangle+\cos \frac{\theta}{2}\left|g_{L} e_{R}\right\rangle
\end{aligned}
$$

of the two-qubit system are appreciably populated, leading to an effective V-system description [see the Appendix] illustrated in Fig. 1(a). Here, $\left|g_{\alpha}\right\rangle$ and $\left|e_{\alpha}\right\rangle$ stand for the ground and excited computational basis states of the left and right qubit ( $\alpha=L, R)$ (also known as site basis states) and $\theta$ is the mixing angle obtained by diagonalizing the two-qubit Hamiltonian (14) in the basis $\left\{\left|e_{L} g_{R}\right\rangle,\left|g_{L} e_{R}\right\rangle\right\}$. Note that state $|a\rangle$ is higher in energy than state $|b\rangle$, which is consistent with the definitions used by Sun et al. [39] and Jung and Brumer [37]. In addition, each qubit is coupled to a thermal bath maintained at different temperatures $T_{L}$ and $T_{R}$ as shown in Fig. 1(b).

The Hamiltonian of the two-qubit system

$$
\hat{H}_{S}=\frac{1}{2} \omega_{L} \hat{\sigma}_{L}^{z}+\frac{1}{2} \omega_{R} \hat{\sigma}_{R}^{z}+J\left(\hat{\sigma}_{L}^{+} \hat{\sigma}_{R}^{-}+\hat{\sigma}_{L}^{-} \hat{\sigma}_{R}^{+}\right)
$$

is expressed via the jump operators $\hat{\sigma}_{i}^{ \pm}$defined as $\hat{\sigma}_{i}^{-}=$ $\left|g_{i}\right\rangle\left\langle e_{i}\left|, \hat{\sigma}_{i}^{+}=\right| e_{i}\right\rangle\left\langle g_{i}\left|, \hat{\sigma}_{i}^{z}=\right| e_{i}\right\rangle\left\langle e_{i}|-| g_{i}\right\rangle\left\langle g_{i}\right|$. The connection between the coupled qubit model and the V-system can be established by diagonalizing the Hamiltonian $\hat{H}_{S}$ in Eq. (14) in the site basis to give $E_{g}=-\frac{1}{2} \bar{\Omega}, E_{a, b}= \pm \frac{1}{2} \sqrt{\tilde{\Delta}^{2}+4 J^{2}}$, and $E_{D}=\frac{1}{2} \bar{\Omega}$. Here, $E_{i}$ and $\left|E_{i}\right\rangle, i=g, a, b$ are the eigenvalues and eigenstates of the V-system $\left(H_{V}=\sum_{i=g, a, b} E_{i}\left|E_{i}\right\rangle\left\langle E_{i}\right|\right)$, $E_{i}$ are arranged in the order of increasing energy, with $E_{g}$ being the ground-state energy), $\bar{\Omega}=\frac{1}{2}\left(\omega_{1}+\omega_{2}\right)$, and $\tilde{\Delta}=$ $\omega_{1}-\omega_{2}$. The V-system parameters, such as the excited-state energy splitting $\Delta$, and the energy difference between the ground and excited states $\omega_{0}$ are obtained as

$$
\begin{aligned}
\Delta & =E_{a}-E_{b}=\sqrt{\tilde{\Delta}^{2}+4 J^{2}}=\sqrt{\left(\omega_{1}-\omega_{2}\right)^{2}+4 J^{2}}, \\
\omega_{0} & =E_{a}-E_{g}=\frac{1}{2} \sqrt{\tilde{\Delta}^{2}+4 J^{2}}+\frac{1}{2} \bar{\Omega} \\
& =\frac{1}{2} \sqrt{\left(\omega_{1}-\omega_{2}\right)^{2}+4 J^{2}}+\frac{1}{4}\left(\omega_{1}+\omega_{2}\right) .
\end{aligned}
$$

For a symmetric V-system, we have $\omega_{1}=\omega_{2}=\omega, \bar{\Omega}=\omega$, and $\tilde{\Delta}=0$, and these equations are further simplified as

$$
\begin{aligned}
\Delta & =E_{a}-E_{b}=2 J, \\
\omega_{0} & =E_{a}-E_{g}=J+\frac{1}{2} \omega .
\end{aligned}
$$


Consider now the two-qubit system coupled to two independent thermal baths maintained at different temperatures, as shown in Fig. 1(b). The system-bath coupling is given by

$$
\hat{H}_{S B}=\sum_{\boldsymbol{k}_{L}, \lambda_{L}} g_{\boldsymbol{k}_{L} \lambda_{L}} \hat{\sigma}_{L}^{+} \hat{b}_{\boldsymbol{k}_{L} \lambda_{L}}+\sum_{\boldsymbol{k}_{R}, \lambda_{R}} g_{\boldsymbol{k}_{R} \lambda_{R}} \hat{\sigma}_{R}^{+} \hat{b}_{\boldsymbol{k}_{R} \lambda_{R}}+\text { H.c. }
$$

This Hamiltonian describes the setup, where the left qubit (denoted $L$ ) is coupled only to the left bath $B_{L}$ and the right qubit $R$ only to the right bath $B_{R}$. A hot bath with $T_{L}=5800 \mathrm{~K}$ is responsible for the incoherent pumping of the left qubit coupled to it.

A cold bath with $T_{R}=0$ accounts for spontaneous emission due to the coupling of the right qubit to the vacuum modes of the electromagnetic field. As shown in the Appendix, the nonequilibrium transport of radiative energy through the two-qubit system can be described by the $B R$ master equations, which are equivalent to those describing the $V$-system, where the transitions $g \rightarrow a$ and $g \rightarrow b$ are driven simultaneously by the left and right baths. (The only assumption made in the derivation is that the population of the doubly excited state $\left|e_{R} e_{L}\right\rangle$ can be neglected). The BR equations take the following form in the eigenstate basis of the system $\{|a\rangle,|b\rangle,|g\rangle\}$ [see Appendix A 2]:

$$
\begin{aligned}
\dot{\rho}_{a a}= & 2 \Gamma_{a a}^{+}\left(\varepsilon_{a}\right) \rho_{g g}-2 \Gamma_{a a}^{-}\left(\varepsilon_{a}\right) \rho_{a a}-\Gamma_{a b}^{-}\left(\varepsilon_{b}\right) \rho_{a b}-\Gamma_{b a}^{-}\left(\varepsilon_{b}\right) \rho_{b a}, \\
\dot{\rho}_{b b}= & 2 \Gamma_{b b}^{+}\left(\varepsilon_{b}\right) \rho_{g g}-2 \Gamma_{b b}^{-}\left(\varepsilon_{b}\right) \rho_{b b}-\Gamma_{a b}^{-}\left(\varepsilon_{a}\right) \rho_{a b}-\Gamma_{b a}^{-}\left(\varepsilon_{a}\right) \rho_{b a}, \\
\dot{\rho}_{a b}= & {\left[\Gamma_{b a}^{+}\left(\varepsilon_{a}\right) \rho_{g g}-\Gamma_{b a}^{-}\left(\varepsilon_{a}\right) \rho_{a a}\right]+\left[\Gamma_{b a}^{+}\left(\varepsilon_{b}\right) \rho_{g g}-\Gamma_{b a}^{-}\left(\varepsilon_{b}\right) \rho_{b b}\right] } \\
& -i \Delta \rho_{a b}-\left[\Gamma_{a a}^{-}\left(\varepsilon_{a}\right)+\Gamma_{b b}^{-}\left(\varepsilon_{b}\right)\right] \rho_{a b},
\end{aligned}
$$

where $\varepsilon_{i}=E_{i}-E_{g}$ is the energy difference between the excited state $|i\rangle$ and the ground state $|g\rangle$, and $\Delta=E_{a}-E_{b}$ is the excited state splitting.

For the V-system with orthogonal transition dipole moment vectors $\left(\boldsymbol{\mu}_{g a} \perp \boldsymbol{\mu}_{g b}\right)$ such as atomic Ca [29] discussed in Sec. IV, the dissipation rates $\Gamma_{i j}^{ \pm}$can be evaluated as described in Appendix A 3 in terms of the incoherent driving and spontaneous decay rates $\gamma_{i j}^{\alpha}$ and $r_{i j}^{\alpha}(i=a, b)$ induced by the $\alpha$ th bath.

$$
\begin{aligned}
\dot{\rho}_{a a}= & r_{a a}^{L} \rho_{g g}-\left(r_{a a}^{L}+\gamma_{a a}^{L}+\gamma_{a a}^{R}\right) \rho_{a a}-\frac{1}{2} \sqrt{r_{a a}^{L} r_{b b}^{L}}\left(\rho_{a b}+\rho_{b a}\right), \\
\dot{\rho}_{b b}= & r_{b b}^{L} \rho_{g g}-\left(r_{b b}^{L}+\gamma_{b b}^{L}+\gamma_{b b}^{R}\right) \rho_{b b}-\frac{1}{2} \sqrt{r_{a a}^{L} r_{b b}^{L}}\left(\rho_{a b}+\rho_{b a}\right), \\
\dot{\rho}_{a b}= & \sqrt{r_{a a}^{L} r_{b b}^{L}} \rho_{g g}-\frac{1}{2} \sqrt{r_{a a}^{L} r_{b b}^{L}}\left(\rho_{a a}+\rho_{b b}\right)-i \Delta \rho_{a b} \\
& -\frac{1}{2}\left(r_{a a}^{L}+r_{b b}^{L}+\gamma_{a a}^{L}+\gamma_{b b}^{L}+\gamma_{a a}^{R}+\gamma_{b b}^{R}\right) \rho_{a b},
\end{aligned}
$$

where $\gamma_{i i}^{\alpha}$ are the spontaneous decay rates of excited level $|i\rangle$ into the $\alpha$-th bath $(\alpha=L, R)$ and $r_{i i}^{\alpha}=\bar{n}_{L} \gamma_{i i}^{\alpha}$ are the corresponding incoherent pumping rates (note that $r_{i i}^{R}=0$ because $T_{R}=0$ by construction).

Importantly, Eqs. (19) are identical to the BR equations (1) with $\gamma_{i}=\gamma_{i i}^{L}+\gamma_{i i}^{R}$ and $r_{i}=r_{i i}^{L}=\bar{n}_{L} \gamma_{i i}^{L}$ (see Appendix A 5). This shows that the overall spontaneous emission rate from the excited state $|i\rangle(i=a, b)$ of the $\mathrm{V}$-system is composed of the contributions due to the left and right baths. While the same is generally true for the absorption rates $r_{i}$, in our specific case, these rates only contain the contribution due to the left bath because the right bath is maintained at zero temperature $\left(\bar{n}_{R}=0\right)$ and thus cannot induce stimulated absorption. Figures 1(b) and 1(c) illustrate the equivalence between the two-qubit system and a V-system, in which each of the transitions $g \leftrightarrow a$ and $g \leftrightarrow b$ is driven simultaneously by the left and right baths. Note that because the baths are uncorrelated, their contributions to the overall transition rates in the V-system are additive (see Appendix).

The transport model gives the ratio of incoherent driving and spontaneous decay rates $r_{i} / \gamma_{i}=r_{i i}^{L} /\left(\gamma_{i i}^{L}+\gamma_{i i}^{R}\right)$ at odds with the equilibrium fluctuation-dissipation theorem, based on which one would expect $r_{i} / \gamma_{i}=r_{i i}^{L} / \gamma_{i i}^{L}=\bar{n}_{L}$. As discussed above and in Refs. [10,27], it is this imbalance between incoherent driving and spontaneous decay that leads to the formation of coherent NESS.

To further characterize nonequilibrium transport in our model two-qubit system, we calculate the energy flux from the "hot" left qubit $L$ to the "cold" right qubit $R[37,39,46]$

$$
\begin{aligned}
\mathcal{J}_{L-R} & =-i\left\langle\left[\hat{\sigma}_{L}^{z}, \hat{H}_{S}\right]\right\rangle=-i 2 J \operatorname{Tr}[\rho(|a\rangle\langle b|-| b\rangle\langle a|)] \\
& =4 J \operatorname{Im}\{\langle a|\rho| b\rangle\},
\end{aligned}
$$

where $\hat{H}_{S}$ is given by Eq. (14), $J$ is the inter-qubit coupling and the trace is taken over the bath degrees of freedom. Equation (20) establishes that the energy flux from qubit $L$ to qubit $R$ is proportional to the imaginary part of Fano coherence in the energy eigenstate basis (13) of the dimer [37,38]. This clarifies the physical significance of steady-state Fano coherences as being responsible for radiative energy transfer from sunlightdriven qubit $L$ to qubit $R$ that is coupled to the vacuum modes of the electromagnetic field. Note that Jung and Brumer [37] define the interqubit flux in a slightly different way using the time evolution of the operator $\left|e_{L}\right\rangle\left\langle e_{L}\right|$ instead of $\hat{\sigma}_{L}^{z}$, which results in their expression being two times smaller than Eq. (20).

We note that while the two-qubit system shown in Fig. 1 acts as a mediator of the thermal energy transfer between the two baths, it cannot be thought of as a quantum heat engine. Indeed, for a quantum system to act like one, there should be a third "work" bath coupled to the system in addition to the hot and cold baths [48], and the system-bath interaction Hamiltonian must be nonlinear. The work extracted from the hot bath is usually described in terms of a time-dependent external field [48]. This situation is different from that considered in this work, where there is no external coherent field, and thus no external work done by the V-system.

To further illustrate this point, consider two coupled identical qubits in the site basis, where the energy flux transferred between the qubits is given by $\mathcal{J}_{L-R}$. The relation between the energy transfer rates $\mathcal{J}_{L-R}, \mathcal{J}_{L-B_{L}}$ (from the hot bath to the left qubit) and $\mathcal{J}_{R-B_{R}}$ (from the right qubit to the cold bath), can be obtained from the master equations for the excited-state populations of the two qubits in the Heisenberg picture [39]

$$
\begin{aligned}
& \dot{\rho}_{e_{L} e_{L}}=\mathcal{J}_{L-R}+\mathcal{J}_{L-B_{L}}, \\
& \dot{\rho}_{e_{R} e_{R}}=\mathcal{J}_{L-R}+\mathcal{J}_{R-B_{R}} .
\end{aligned}
$$

In the steady state, the rates of change of the excited-state populations vanish $\left(\dot{\rho}_{e_{L} e_{L}}=\dot{\rho}_{e_{R} e_{R}}=0\right)$, and Eqs. (21) and (22) give

$$
\left|\mathcal{J}_{L-B_{L}}\right|=\left|\mathcal{J}_{L-R}\right|=\left|\mathcal{J}_{R-B_{R}}\right|
$$


The above equation shows that the energy transfer rates between the thermal baths and the two qubits are equal in magnitude, which is consistent with the principle of conservation of energy. This implies that the two-qubit system acts as a "mediator" of nonequilibrium energy transfer between the two baths, rather than as a quantum heat engine.

As shown above [Eq. (20)], the energy flux from qubit $L$ to qubit $R$ is proportional to the imaginary part of Fano coherence in the energy eigenstate basis $\operatorname{Im}\left[\rho_{a b}\right]$. By inspecting the BR equations (1), we note that $\operatorname{Im}\left[\rho_{a b}\right]$ can only be generated from $\operatorname{Re}\left[\rho_{a b}\right]$ via unitary evolution under the system Hamiltonian [caused by the term $-i \Delta \rho_{a b}$ ] provided that $\Delta>0$.

It also follows from the BR equations (1) that the real part of Fano coherence is generated by the term $\sqrt{r_{a} r_{b}} \rho_{g g}$, which can be written as

$$
\mathcal{I}_{a b}=\sqrt{\mathcal{I}_{a} \mathcal{I}_{b}}
$$

where $\mathcal{I}_{a}=r_{a} \rho_{g g}$ and $\mathcal{I}_{b}=r_{b} \rho_{g g}$ are the incoherent absorption fluxes corresponding to the $g \rightarrow a$ and $g \rightarrow b$ transitions in the $\mathrm{V}$-system driven by the left bath.

Thus, the physical significance of the imaginary part of Fano coherence $\operatorname{Im}\left[\rho_{a b}\right]$ is that it represents a relative phase between the eigenstates $|a\rangle$ and $|b\rangle$ created via quantum interference of incoherent absorption fluxes $\mathcal{I}_{a}$ and $\mathcal{I}_{b}$ followed by coherent evolution of the $\mathrm{V}$-system with $\Delta>0$, which interconverts $\operatorname{Re}\left[\rho_{a b}\right]$ and $\operatorname{Im}\left[\rho_{a b}\right]$ [26]. Significantly, the generation of $\operatorname{Im}\left[\rho_{a b}\right]$ is a two-step process, and both of the steps are essential. For example, in the degenerate V-system $(\Delta=J=0)$, no interconversion occurs between $\operatorname{Re}\left[\rho_{a b}\right]$ and $\operatorname{Im}\left[\rho_{a b}\right]$, and the imaginary part of Fano coherence vanishes at all times [25]. This is consistent with Eq. (20), which predicts zero energy flux between the left and right qubits in the absence of coherent coupling $(J=0)$.

Equation (23) suggests that the magnitude of the steadystate energy flux across the two-qubit system $\mathcal{J}_{L-R}$ is equal to that of the spontaneous emission flux from the right qubit to the right bath $\mathcal{J}_{R-B_{R}}$. Using the relation between the real and imaginary parts of Fano coherence in the steady state $\rho_{a b}^{I}=$ $-\frac{\Delta}{r+\gamma} \rho_{a b}^{R}$ [see the discussion below Eq. (6)], we obtain

$$
\left|\mathcal{J}_{L-R}\right|=\frac{4 J \Delta}{r+\gamma}\left|\rho_{a b}^{R}\right|=\frac{8 J^{2}}{r+\gamma}\left|\rho_{a b}^{R}\right| .
$$

Thus the steady-state flux only depends on the real part of Fano coherence. This is consistent with the physical interpretation developed above, which attributes the flux to the interference between incoherent excitations $g \rightarrow a$ and $g \rightarrow$ $b$ generated at the same time. These excitations create an in-phase superposition $\left|\phi_{+}\right\rangle=\frac{1}{\sqrt{2}}(|a\rangle+|b\rangle)$ characterized by $\rho_{a b}^{R} \neq 0$, which then evolves and decays in time [17]. Transport in the localized (site) basis can then be interpreted as an interplay between the formation and decay of the delocalized coherent superposition $\left|\phi_{+}\right\rangle$via incoherent pumping from the ground state.

Finally, we note that it is possible to express the flux (25) via the deviation of excited-state populations from thermodynamic equilibrium using Eq. (8)

$$
\left|\mathcal{J}_{L-R}\right|=\frac{8 J^{2}}{r+\gamma} \frac{\left|\rho_{a a}^{(\mathrm{eq})}-\rho_{a a}\right|}{\rho_{a a}^{(\mathrm{eq})}} .
$$

This result shows that the nonequilibrium flux vanishes under isotropic excitation, where $\rho_{a a}=\rho_{a a}^{(\mathrm{eq})}$, as expected in thermodynamic equilibrium [39].

\section{B. Effect of excited-state relaxation and decoherence}

Realistic quantum systems are always subject to environmental perturbations causing relaxation and decoherence. In this section, we explore the robustness of steady-state Fano coherences against these detrimental effects. To this end, we follow previous theoretical work $[36,49,50]$ and introduce phenomenological relaxation and decoherence channels represented by the terms $\Gamma \rho_{a a}$ and $\gamma_{d} \rho_{a b}$ in Eq. (2). Following the analysis presented above, we obtain steady-state solutions as a function of relaxation and decoherence rates $\Gamma$ and $\gamma_{d}$ :

$$
\begin{aligned}
\rho_{a a} & =\frac{r\left[\Delta^{2}+\left(\gamma+\gamma_{d}\right)\left(r+\gamma+\gamma_{d}\right)\right]}{(3 r+\gamma+\Gamma)\left[\Delta^{2}+\left(r+\gamma+\gamma_{d}\right)^{2}\right]-3 r^{2}\left(r+\gamma+\gamma_{d}\right)}, \\
\rho_{a b}^{R} & =\frac{r(\gamma+\Gamma)\left(r+\gamma+\gamma_{d}\right)}{(3 r+\gamma+\Gamma)\left[\Delta^{2}+\left(r+\gamma+\gamma_{d}\right)^{2}\right]-3 r^{2}\left(r+\gamma+\gamma_{d}\right)},
\end{aligned}
$$

$\rho_{a b}^{I}=-\frac{\Delta}{\left(r+\gamma+\gamma_{d}\right)} \rho_{a b}^{R}$.

These equations suggest that both the real and imaginary steady-state coherences can survive in the presence of relaxation and decoherence. Increasing the relaxation rate does not significantly affect the steady-state coherence in the typical limit $\Gamma \gg r+\gamma$, where the dependence $\rho_{a b}^{R}(\Gamma) \simeq \frac{\gamma+\Gamma}{3 r+\gamma+\Gamma}$ is weak (Ref. [44] considers the effect of relaxation is more detail). We therefore focus on the case of pure decoherence (or dephasing) assuming $\Gamma=0$.

In Fig. 5, we plot the dependence of the steady-state Fano coherence on the excited-state splitting and the reduced decoherence rate $\gamma_{d} / \gamma(27)$. We observe that, regardless of the pumping intensity, the steady-state coherences are maximized for small excited-state splittings $\Delta / \gamma \ll 1$. In the weak pumping limit illustrated in Fig. 5(a) the coherences also decrease rapidly with the decoherence rate, falling below one part in $10^{3}$ for $\gamma_{d} / \gamma>6$. Similar trends are observed in the case of intermediate pumping $(\bar{n}=1)$, even though the absolute magnitude of the steady-state coherence in this regime is larger by two orders of magnitude. The coherences in the strong pumping limit $(\bar{n} \gg 1)$ are large and while they they do not fall off as strongly with $\Delta / \gamma$ they do get significantly suppressed by decoherence. The extent of this suppression appears insensitive to the pumping rate.

Remarkably, as shown in Figs. 5(a)-5(c), the presence of decoherence can enhance the magnitude of steady-state Fano coherences. For instance, at $\Delta / \gamma_{d}=2$ the value of $\rho_{a b}^{R}$ passes through a maximum near $\gamma_{d}=2$ and then decreases again at higher $\gamma_{d}$. This enhancement is particularly pronounced for large $\Delta / \gamma$ in the weak-pumping limit, where we observe up to fivefold coherence enhancements [44]. To further explore this counterintuitive effect, we rewrite Eq. (27) in terms of the dimensionless parameters $\tilde{\gamma}_{d}=\gamma_{d} / \gamma$ and $\tilde{\Delta}=\Delta / \gamma$

$$
\rho_{a b}^{R}=\frac{\bar{n}\left(\bar{n}+1+\tilde{\gamma}_{d}\right)}{(3 \bar{n}+1)\left[\tilde{\Delta}^{2}+\left(\bar{n}+1+\tilde{\gamma}_{d}\right)^{2}\right]-3 \bar{n}^{2}\left(\bar{n}+1+\tilde{\gamma}_{d}\right)} .
$$



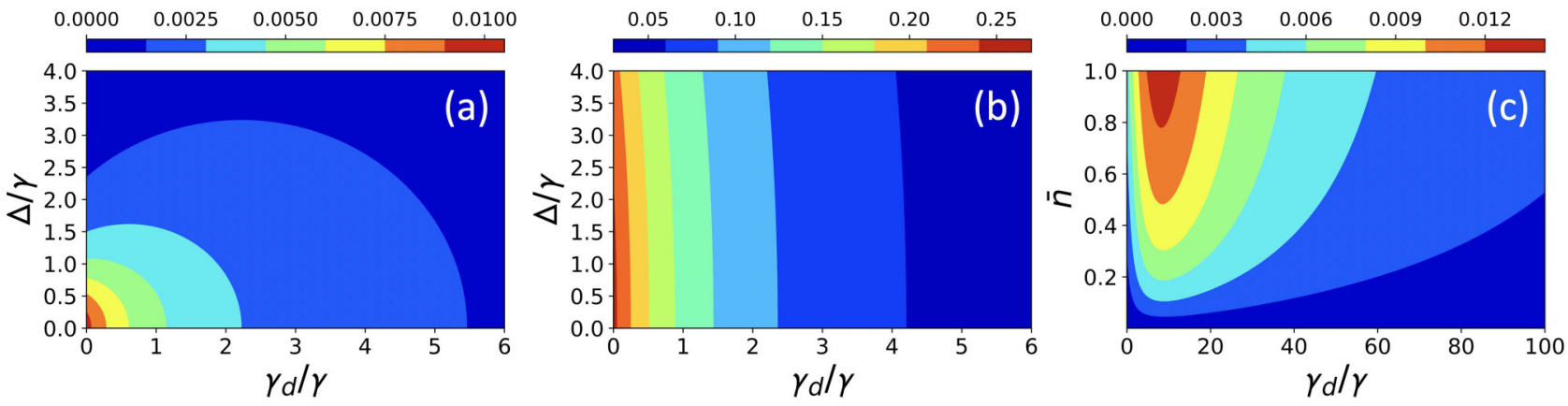

FIG. 5. Contour plots of steady-state Fano coherence as a function of the excited-state splitting $\Delta / \gamma$ and of the decoherence rate $\gamma_{d} / \gamma$ under weak (a) and strong (b) $x$-polarized incoherent pumping. The values of the average photon number used are $\bar{n}=0.01$ (a) and $\bar{n}=100$ (c). (c) shows the steady-state Fano coherence as a function the decoherence rate and $\bar{n}$ at a fixed $\Delta / \gamma=10$.

The roots of the equation $\frac{d}{d \tilde{\gamma}_{d}} \rho_{a b}^{R}=0$ are given by $\tilde{\gamma}_{d}= \pm \tilde{\Delta}-$ $\bar{n}-1$ [44]. Picking the physical root corresponding to $\tilde{\Delta}>0$, we obtain the optimal decoherence rate

$$
\gamma_{d}=\Delta-r-\gamma
$$

that maximizes the value of the steady-state Fano coherence. In the weak pumping regime, Eq. (29) simplifies to $\gamma_{d} \approx \Delta$ which defines the diagonal "line of optimal decoherence" in Figs. 5(a) and 5(b), along which the steady-state Fano coherence takes a maximum value.

Importantly, the maximum of the steady-state Fano coherence as a function of $\gamma_{d}$ only appears when the optimal decoherence rate in Eq. (29) is positive $(\Delta \geqslant r+\gamma)$, which corresponds to the underdamped regime of V-system dynamics $[13,17,26,29]$. This is illustrated in Fig. 5(c), which shows that the maximum of $\rho_{a b}^{R}$ is located along the vertical line of constant $\gamma_{d} / \gamma=10$. Thus the expression $\gamma_{d} \approx \Delta$ is to be interpreted as the limiting case of Eq. (29) in the underdamped regime, outside of which the steady-state coherence shows no maximum as a function of $\gamma_{d}$, and Eq. (29) does not apply.

The population-to-coherence ratio (11) in the presence of dephasing can be obtained from Eq. (27)

$$
\mathcal{C}=\frac{1}{1+\frac{\gamma_{d}}{\gamma}+\frac{\Delta^{2}}{\gamma\left(r+\gamma+\gamma_{d}\right)}} .
$$

The ratio takes the maximum value $\mathcal{C}_{\max }=\frac{1}{1+\gamma_{d} / \gamma+\left(r+\gamma+\gamma_{d}\right) / \gamma}$ when the sum in the denominator is a minimum, which occurs when $\gamma_{d}=\Delta-r-\gamma$. This is the same condition as required for the maximization of the real part of steady-state Fano coherence (29).

Under typical molecular excitation conditions, nonradiative dephasing is fast compared with radiative processes $\left(\gamma_{d} \gg \gamma, r\right)$ and we obtain $\mathcal{C} \simeq \frac{1}{1+\gamma_{d} / \gamma+\Delta^{2} /\left(\gamma \gamma_{d}\right)} \ll 1$. In the limit of small $\Delta \ll \sqrt{\gamma \gamma_{d}}$ the last term in the denominator can be neglected, giving $\mathcal{C} \simeq \frac{1}{1+\gamma_{d} / \gamma} \simeq \gamma / \gamma_{d} \ll 1$. Thus, the ratio of steady-state Fano coherences to excited-state populations will be suppressed by the factor $\gamma_{d} / \gamma$ in the presence of environmental dephasing, as long as the system-environment coupling responsible for the dephasing does not generate any steady-state coherence (as assumed here, see Sec. III).

Figure 6 shows the steady-state coherence-to-population ratio as a function of dephasing. In accordance with the analytic result obtained above (29), we observe the emergence of a maximum at the optimal decoherence rate $\gamma_{d}=$ $\Delta-r-\gamma$. The maximum is only present at sufficiently large excited-state splittings $\Delta$ such that $\Delta-r-\gamma>0$ (underdamped regime of V-system dynamics $[13,18,26]$ ) as shown in Figs. 6(a) and 6(b). Decreasing the value of $\Delta$ brings the Vsystem into the overdamped regime $[13,18,26]$ characterized by $\Delta / \gamma<1$. As shown in Figs. 6(c) and 6(d), this leads to the disappearance of the maximum and the expected monotonic decrease of the $\mathcal{C}$-ratio with increasing decoherence rate.

While the ability of dephasing to enhance steady-state coherence in the underdamped regime is surprising, the dephasing enhanced steady-state coherence can be understood by considering the individual contributing excitations. In the underdamped regime, one mechanism of coherence loss arises due to the cancellation of excitations at different times that carry different phases due to their unitary evolution in the excited state manifold [see Fig. 3(b)]. By introducing an explicit dephasing channel, excitations generated at earlier times lose more coherences than those generated later. As a result,
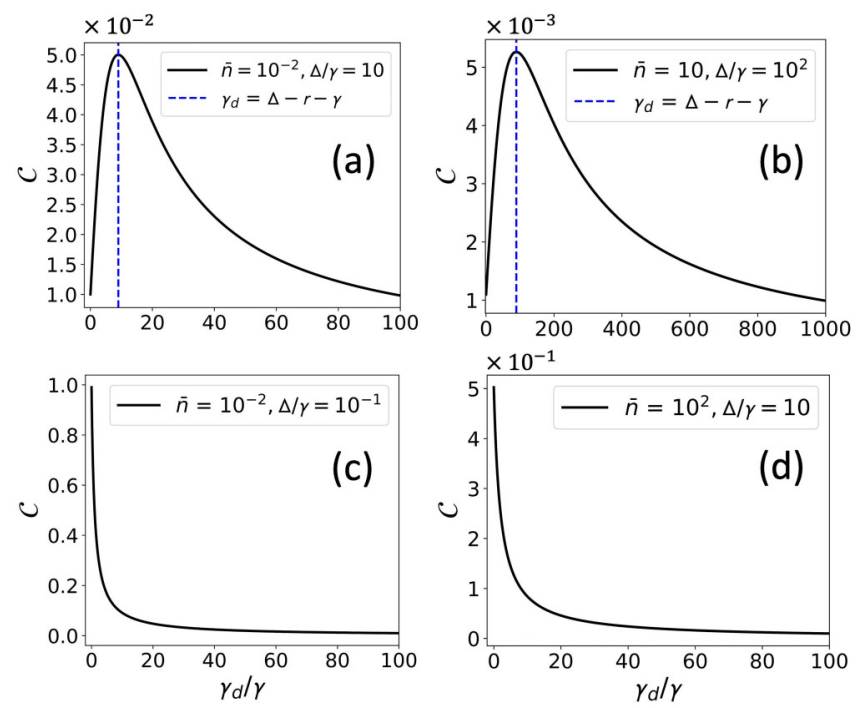

FIG. 6. Steady-state coherence-to-population ratio $\mathcal{C}$ plotted as a function of dimensionless decoherence rate $\gamma_{d} / \gamma$ in the weakpumping limit [(a) and (c)] and in the strong-pumping limit [(b) and (d)]. The values of the $\Delta / \gamma$ are indicated in each panel. 
these excitations do not destructively interfere as much as they could in the absence of dephasing. The dephasing then weights the phase average towards a narrower window of excitation times, decreasing the ensemble-induced dephasing process. However, dephasing leads to coherence loss at the level of individual excitations. Consequently, this trade-off leads to a competition between the two effects of increased dephasing, allowing for dephasing-enhanced steady-state coherences in certain regimes when the decreased ensemble dephasing outweighs the increased excitation level dephasing.

We conclude that in the underdamped (or large- $\Delta$ ) regime of V-system dynamics, steady-state Fano coherence are maximized when the decoherence rate is equal to the energy splitting between the excited states. The physical origin of the maximization can be interpreted at the level of individual incoherent excitations, whose unitary evolution is accompanied by environmental decoherence. The competition of these mechanisms can result in an enhancement of steady-state coherence through minimization of destructive interference caused by coherent evolution. A similar mechanism is at play in environment-assisted quantum transport, whereby the addition of a moderate amount of noise positively affects the energy flux in multichromophoric reaction complexes $[46,47,49-51]$. The decoherence-induced enhancement of Fano coherences could be used to facilitate their experimental observation via measuring excited-state populations, as discussed in the following section.

\section{EXPERIMENTAL DETECTION OF STEADY-STATE FANO COHERENCE}

In our previous work [29], we proposed a scheme for the experimental observation of transient Fano coherences by detecting the fluorescence signal emitted by incoherently pumped Calcium atoms. This scheme suffers from two difficulties: First, the transient nature of noise-induced coherent dynamics makes it sensitive to the turn-on time of the radiation. In particular, Fano coherences are known to vanish in the limit where the turn-on time is much longer than that of spontaneous emission [18]. Second, because the total fluorescence intensity is independent of Fano coherence [29], it required the detection of the fluorescence emitted into a specific range of solid angles.

In this section, we eliminate these difficulties by arguing that steady-state Fano coherences can be observed by measuring the total steady-state fluorescence signal emitted by Calcium atoms irradiated by polarized versus isotropic incoherent light. Unlike the method proposed in Ref. [29], the new detection scheme requires neither rapid turn-on of the radiation field nor spatially resolved detection of the fluorescence signal.

Our proposed experimental setup is similar to that described in our previous work (see Fig. 1(a) and Fig. 1 of Ref. [29]) and consists of a beam of $\mathrm{Ca}$ atoms initially prepared in the ground ${ }^{1} \mathrm{~S}_{0}$ electronic state. The atoms are continuously irradiated with spectrally broadened light polarized in the $x$-direction, inducing the electric dipole transition ${ }^{1} \mathrm{~S}_{0} \rightarrow{ }^{1} \mathrm{P}_{m_{J}}$ from the $J=0$ ground state to $J=1$ excited states with $m_{J}= \pm 1$. The excited states are split by an external magnetic field pointing in the $z$ direction, forming a nearly perfect V-system with tunable excited-state splitting $\Delta$ [29]. Following initial turn-on of incoherent excitation, a fraction of $\mathrm{Ca}$ atoms in the beam will be excited to the ${ }^{1} \mathrm{P}_{m_{J}}$ states. The excited atoms will decay to the ground state emitting fluorescence photons.

The average intensity of the radiation emitted by $\mathrm{Ca}$ atoms in the direction $(\theta, \phi)$ at a distance $R$ away from the source is [29]

$$
\begin{aligned}
\langle I(\mathbf{R}, t)\rangle= & \frac{n_{r} \omega_{0}^{4}}{32 \pi^{2} \epsilon_{0} c^{3} R^{2}}\left[\frac{1+\cos ^{2} \theta}{2}\left(\rho_{a a}\left(t^{\prime}\right)+\rho_{b b}\left(t^{\prime}\right)\right)\right. \\
& \left.+\sin ^{2} \theta\left(\cos 2 \phi \rho_{a b}^{R}\left(t^{\prime}\right)-\sin 2 \phi \rho_{a b}^{R}\left(t^{\prime}\right)\right)\right],
\end{aligned}
$$

where $t^{\prime}=t+R / c$ is the retarded time, $n_{r}$ is the refractive index of the medium, $c$ is the speed of light in free space. Integrating Eq. (31) over all spatial directions $(\theta, \phi)$ we obtain the total emitted intensity $I=\frac{8 \pi}{3} I^{0}\left(\rho_{a a}+\rho_{b b}\right)$, where $I^{0}=$ $n_{r} \omega_{0}^{4} /\left(32 \pi^{2} \epsilon_{0} c^{3} R^{2}\right)$. For a symmetric V-system $\rho_{a a}=\rho_{b b}$, this expression further simplifies to

$$
I=\frac{16 \pi}{3} I^{0} \rho_{a a} .
$$

Importantly, unlike its spatially resolved counterpart (31) the integrated fluorescence intensity depends only on the excited state population and not on the Fano coherence. Therefore, in order to directly observe transient Fano coherences, it is necessary to measure the intensity emitted into specific domains of space [29] in such a way that the partially integrated fluorescence intensity is made to explicitly depend of Fano coherence terms $\rho_{a b}$.

Here, we show that it is not necessary to implement spatially resolved fluorescence detection [29] to observe steady-state Fano coherences in a V-system excited by polarized incoherent light. As shown in Sec. III above, the magnitude of the real part of steady-state Fano coherence is directly related to the deviation of excited-state populations from their values in thermodynamic equilibrium via Eq. (9). As such, observing these deviations could be used to directly probe steady-state Fano coherences.

More specifically, let the intensity of the emitted light in the absence of Fano coherence be $I^{(0)}$. This intensity corresponds to the excited-state population in the absence of Fano coherence given by Eq. (7). From Eqs. (8) and (32), the relative difference of the emitted light intensity with and without steady-state Fano coherence is

$$
\frac{\Delta I}{I^{0}}=\frac{\rho_{a a}^{(\mathrm{eq})}-\rho_{a a}}{\rho_{a a}^{(\mathrm{eq})}}=\rho_{a b}^{R}
$$

We see that in the coherent NESS induced by $x$-polarized incoherent driving of the Calcium V-system, excited-state populations are suppressed compared to the canonical Boltzmann distribution. The intensity of the light emitted by the Calcium atoms driven into the NESS is therefore diminished compared to the intensity that would be emitted by the atoms in coherence-free canonical thermal equilibrium (attainable by isotropic incoherent driving $[13,17,29])$. The relative difference in the fluorescence signals emitted by the atoms driven by $x$-polarized versus isotropic incoherent light can therefore 

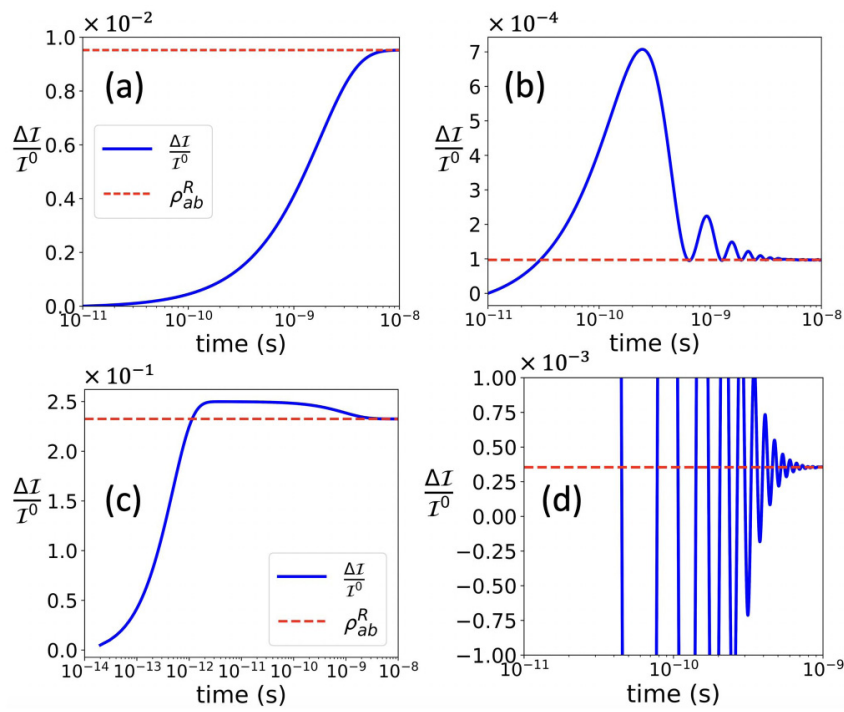

FIG. 7. (a) The ratio of fluorescence intensities calculated for $\mathrm{Ca}$ atoms driven by $x$-polarized vs isotropic incoherent light in different regimes: (a) $\bar{n}=10^{-2}$ and $\Delta / \gamma=10^{-1}$; (b) $\bar{n}=10^{-2}$ and $\Delta / \gamma=$ 10; (c) $\bar{n}=10^{3}$ and $\Delta / \gamma=10$; (d) $\bar{n}=10$ and $\Delta / \gamma=10^{2}$. The spontaneous decay lifetime used in the calculations is $\tau_{s}=1 / \gamma=$ $10^{-9} \mathrm{~s}$

be interpreted as an experimental signature of steady-state Fano coherences.

Figure 7 shows the experimentally relevant fluorescence difference signals calculated by numerical integration of the $\mathrm{BR}$ equations. We observe rich transient dynamics, after which the signals reach their nonequilibrium steady-state values consistent with Eq. (33). The observable relative intensity difference remains fairly small $(<1 \%)$ in the weak-pumping limit but grows significantly with the pumping intensity, reaching $23 \%$ for $\bar{n}=10^{3}$ and $\Delta / \gamma=10$. As pointed out in Sec. III B above, it might be possible to further enhance the steady-state Fano coherence (and hence the fluorescence difference signal) by inducing moderate decoherence between the excited states of the calcium atoms. This could be achieved experimentally by, e.g., adding magnetic field noise or isolating the atoms in a rare-gas host matrix [52-54].

\section{SUMMARY AND CONCLUSIONS}

We have presented closed-form analytical solutions to the $\mathrm{BR}$ quantum master equations for a three-level V-system driven by polarized incoherent radiation, which reveal the presence of NESS featuring substantial Fano coherences in the energy eigenstate basis. As pointed out in [27], the coherent NESS emerge as a result of an imbalance between polarized incoherent excitation and spontaneous emission. The imbalance occurs due to the directional nature of polarized incoherent pumping [29], whereby only a single polarization mode of the radiation field interacts with the $\mathrm{V}$ system [10]. As a result, polarized absorption rates are smaller by the factor of $16 \pi / 3$ than their isotropic counterparts (see Appendix A 4), while the rates of spontaneous emission remain the same as in the case of unpolarized (isotropic) incoherent pumping, where both polarization modes participate in the emission process. The rates of isotropic incoherent pumping $r$ and spontaneous emission $\gamma$ are balanced, i.e., related by the equilibrium fluctuation-dissipation theorem $r=\bar{n} \gamma$, and thus no steady-state Fano coherences survive in the V-system driven by unpolarized incoherent light [27].

The imbalance between absorption and spontaneous emission that leads to the emergence of steady-state Fano coherences can be thought of as originating from an additional, symmetry-breaking bath, leading to a formal analogy between the V-system driven by polarized incoherent light and a nonequilibrium transport problem involving a system of two qubits coupled to two thermal baths, one at $T=5800 \mathrm{~K}$ responsible for incoherent solar excitation [see Fig. 1(b)] and the other at zero temperature representing spontaneous emission. We provide a detailed derivation of the master equation for the open two-qubit system [see the Appendix], which establishes its equivalence to the BR equation for the V-system [Eqs. (18) and (19)], whose transitions are driven simultaneously by linearly polarized incoherent light (left bath) and a zero-temperature thermal field (right bath). The derivation is valid in the weak-coupling limit, where the population of the doubly excited eigenstate of the two-qubit system can be neglected. We further show that in this two-bath model, the imaginary part of the coherence between the quasi-degenerate excited states in the V-system has a special significance: it is proportional to the nonequilibrium flux between the two-level systems coupled to hot and cold baths.

We note that our results could be relevant to quantum thermodynamics of heat transport between two reservoirs via coupled intermediary systems, which has been considered in several recent papers [55-57]. While the case of two coupled qubits (sometimes referred to as a "quantum entangler" [57]) is not the main focus of these papers, they address issues that are very close to those considered here.

Our steady-state analysis demonstrates a remarkable relation between steady-state Fano coherences and the deviation of excited-state populations from thermal equilibrium: $\Delta \rho_{a a} / \rho_{a a}^{(c)}=\rho_{a b}^{R}$. This relation suggests that signatures of steady-state Fano coherences could be observed through measuring excited-state populations. We further extend our steady-state analysis to include environmental decoherence and relaxation effects, finding that steady-state Fano coherences persist in the V-system illuminated by polarized incoherent radiation even in the presence of decoherence and relaxation effects.

While steady-state Fano coherences are generally suppressed by environmental relaxation and decoherence (see Sec. III B), we find that the effect of decoherence can be more subtle. In particular, the real part of the steady-state Fano coherence can be enhanced by a moderate amount of excited-state decoherence, as shown in Fig. 5. The magnitude of the steady-state Fano coherence is maximized when the decoherence rate is equal to the excited energy splitting minus the combined rates of radiative pumping and spontaneous decay $\left(\gamma_{d}=\Delta-r-\gamma\right)$. We suggest that this counterintuitive enhancement of steady-state Fano coherence occurs due to a suppression of destructive interference of incoherent excitations generated at different times.

Finally, we offer an improved method for the detection of steady-state Fano coherences using Calcium atoms 
irradiated by polarized incoherent light. Specifically, we propose to detect the signatures of Fano coherences by measuring the deviations of excited-state populations in the NESS from their equilibrium values (obtained by pumping the atoms with isotropic incoherent light). Unlike the scheme proposed in our previous work [29], this detection scenario only relies on the total fluorescence intensity measurements, and requires neither spatially resolved fluorescence detection nor rapid turn-on of the incoherent light.

\section{ACKNOWLEDGMENTS}

This work was partially supported by the NSF (grant PHY1912668). P.B. acknowledges support from the U.S. Air Force office of Scientific Research (AFOSR) under Contract No. FA9550-17-1-0310. We thank Amar Vutha and Jonathan Weinstein for stimulating discussions.

\section{APPENDIX}

In this Appendix, we will derive the Bloch-Redfield (BR) quantum master equations for the system of two coupled qubits interacting with two independent thermal baths, one of which represents a beam of incoherent radiation field at a finite temperature polarized in the $x$ direction, and another is responsible for (isotropic) spontaneous emission. We show that the resulting BR master equations [Eqs. (18) of the main text] are equivalent to those describing the $\mathrm{V}$-system driven by $x$-polarized incoherent light [Eq. (19) of the main text] under the assumption that transitions to and from the doubly excited state of the two-qubit system can be neglected. As shown in Appendix A 1 below and illustrated in Fig. 1(c) of the main text, each of the transitions in the V-system $(g \leftrightarrow a$ and $g \leftrightarrow b$ ) is driven simultaneously by both the left and right baths. Finally, we present the expressions for the incoherent pumping and spontaneous emission rates of the V-system in terms of the corresponding rates due to the left and right baths, thereby completing the derivation of Eqs. (19) of the main text.

\section{Interaction Hamiltonian and system-bath couplings}

To derive the BR equations [Eq. (18) of the main text] in the eigenstate basis for a system of two coupled qubits interacting with two independent thermal baths, we begin with the twoqubit Hamiltonian

$$
\hat{H}_{S}=\frac{1}{2} \omega_{L} \hat{\sigma}_{L}^{z}+\frac{1}{2} \omega_{R} \hat{\sigma}_{R}^{z}+J\left(\hat{\sigma}_{L}^{+} \hat{\sigma}_{R}^{-}+\hat{\sigma}_{L}^{-} \hat{\sigma}_{R}^{+}\right),
$$

where $\left|e_{\alpha}\right\rangle,\left|g_{\alpha}\right\rangle$ ( $\alpha=L, R$ for left and right) are the excited and ground basis states of the individual qubit $\alpha$ (also known as the site or bare basis states), $\hat{\sigma}_{\alpha}^{+}=\left|e_{\alpha}\right\rangle\left\langle g_{\alpha}\left|, \hat{\sigma}_{\alpha}^{-}=\right| g_{\alpha}\right\rangle\left\langle e_{\alpha}\right|$ are the raising and lowering operators, $J$ is the coupling between the qubits and $\hat{\sigma}_{\alpha}^{z}=\left|e_{\alpha}\right\rangle\left\langle e_{\alpha}|-| g_{\alpha}\right\rangle\left\langle g_{\alpha}\right|$.

The interaction of the two-qubit system with independent heat baths is described by the Hamiltonian expressed in the site basis

$$
\hat{H}_{S B}=\sum_{\boldsymbol{k}_{L}, \lambda_{L}} g_{\boldsymbol{k}_{L} \lambda_{L}} \hat{\sigma}_{L}^{+} \hat{b}_{\boldsymbol{k}_{L} \lambda_{L}}+\sum_{\boldsymbol{k}_{R}, \lambda_{R}} g_{\boldsymbol{k}_{R} \lambda_{R}} \hat{\sigma}_{R}^{+} \hat{b}_{\boldsymbol{k}_{R} \lambda_{R}}+\text { H.c., }
$$

where $g_{\boldsymbol{k}_{\alpha} \lambda_{\alpha}}$ are the system-bath coupling coefficients $(\alpha=$ $L, R)$. We assume that qubit $\alpha$ interacts only with the $\alpha$-th bath. In this section, we will leave the coupling coefficients $g_{k_{\alpha} \lambda_{\alpha}}$ unspecified to keep the discussion general. Below, we will specialize to the case of incoherent radiation field.

Our goal is to transform the interaction Hamiltonian (A2) from the site basis to the energy eigenstate basis and to show how it maps onto the interaction Hamiltonian for the V-system in the weak coupling limit. We start by expressing the bare state basis in Eq. (A2) in the full 4-dimensional Hilbert space. The two coupled qubits interacting with two independent heat baths can be represented in this space by the bare states $\left|e_{L} e_{R}\right\rangle$, $\left|e_{L} g_{R}\right\rangle,\left|g_{L} e_{R}\right\rangle$, and $\left|g_{L} g_{R}\right\rangle$.

Diagonalization of the system Hamiltonian given by Eq. (A1) gives the eigenstates of the two-qubit system, which are related to the bare states as

$$
\begin{gathered}
|D\rangle=\left|e_{L} e_{R}\right\rangle, \\
|a\rangle=\cos \frac{\theta}{2}\left|e_{L} g_{R}\right\rangle+\sin \frac{\theta}{2}\left|g_{L} e_{R}\right\rangle, \\
|b\rangle=-\sin \frac{\theta}{2}\left|e_{L} g_{R}\right\rangle+\cos \frac{\theta}{2}\left|g_{L} e_{R}\right\rangle, \\
|g\rangle=\left|g_{L} g_{R}\right\rangle,
\end{gathered}
$$

where $\theta$ is the mixing angle [36,58] $(\theta=\pi / 2$ for the symmetric dimer).

From Eqs. (A4) and (A5), we obtain for $\left|e_{L} g_{R}\right\rangle$ and $\left|g_{L} e_{R}\right\rangle$

$$
\begin{aligned}
& \left|e_{L} g_{R}\right\rangle=\cos \frac{\theta}{2}|a\rangle-\sin \frac{\theta}{2}|b\rangle, \\
& \left|g_{L} e_{R}\right\rangle=\sin \frac{\theta}{2}|a\rangle+\cos \frac{\theta}{2}|b\rangle .
\end{aligned}
$$

Using Eqs. (A3), (A6), (A7), and (A8), we transform the qubit jump operators in the site basis to the energy eigenstate basis

$$
\begin{aligned}
\sigma_{L}^{+} \otimes I_{R}=\left|e_{L}\right\rangle\left\langle g_{L}\right| \otimes I_{R} & =\left|e_{L}\right\rangle\left\langle g_{L}\left|\otimes\left(\left|g_{R}\right\rangle\left\langle g_{R}|+| e_{R}\right\rangle\left\langle e_{R}\right|\right)=\right| e_{L} g_{R}\right\rangle\left\langle g_{L} g_{R}|+| e_{L} e_{R}\right\rangle\left\langle g_{L} e_{R}\right| \\
= & {\left[\cos \frac{\theta}{2}|a\rangle-\sin \frac{\theta}{2}|b\rangle\right]\langle g|+| D\rangle\left[\sin \frac{\theta}{2}\langle a|+\cos \frac{\theta}{2}\langle b|\right] } \\
= & \cos \frac{\theta}{2}|a\rangle\left\langle g\left|-\sin \frac{\theta}{2}\right| b\right\rangle\left\langle g\left|+\sin \frac{\theta}{2}\right| D\right\rangle\left\langle a\left|+\cos \frac{\theta}{2}\right| D\right\rangle\langle b|, \\
I_{L} \otimes \sigma_{R}^{+}=I_{L} \otimes\left|e_{R}\right\rangle\left\langle g_{R}\right| & =\left(\left|e_{L}\right\rangle\left\langle e_{L}|+| g_{L}\right\rangle\left\langle g_{L}\right|\right) \otimes\left|e_{R}\right\rangle\left\langle g_{R}\right| \\
& =\left|e_{L} e_{R}\right\rangle\left\langle e_{L} g_{R}|+| g_{L} e_{R}\right\rangle\left\langle g_{L} g_{R}\right|
\end{aligned}
$$




$$
\begin{aligned}
& =|D\rangle\left[\operatorname { c o s } \frac { \theta } { 2 } \left\langlea\left|-\sin \frac{\theta}{2}\langle b|\right]+\left[\sin \frac{\theta}{2}|a\rangle+\cos \frac{\theta}{2}|b\rangle\right]\langle g|\right.\right. \\
& =\cos \frac{\theta}{2}|D\rangle\left\langle a\left|-\sin \frac{\theta}{2}\right| D\right\rangle\left\langle b\left|+\sin \frac{\theta}{2}\right| a\right\rangle\left\langle g\left|+\cos \frac{\theta}{2}\right| b\right\rangle\langle g|,
\end{aligned}
$$

where $I_{\alpha}$ is the unit operator in the Hilbert space of the $\alpha$ th qubit.

On substituting Eqs. (A9) and (A10) to Eq. (A2), we find the system-bath interaction Hamiltonian of two coupled qubits in the energy eigenstate basis

$$
\begin{aligned}
\hat{H}_{S B}= & \sum_{\boldsymbol{k}_{L}, \lambda_{L}} g_{\boldsymbol{k}_{L} \lambda_{L}} \hat{\sigma}_{L}^{+} \otimes I_{R} \hat{b}_{\boldsymbol{k}_{L} \lambda_{L}}+\sum_{\boldsymbol{k}_{R}, \lambda_{R}} g_{\boldsymbol{k}_{R} \lambda_{R}} I_{R} \otimes \hat{\sigma}_{R}^{+} \hat{b}_{\boldsymbol{k}_{R} \lambda_{R}}+\text { H.c. } \\
= & |a\rangle\left\langle g\left|\left\{\sum_{\boldsymbol{k}_{L}, \lambda_{L}} g_{\boldsymbol{k}_{L} \lambda_{L}} \hat{b}_{\boldsymbol{k}_{L} \lambda_{L}} \cos \frac{\theta}{2}+\sum_{\boldsymbol{k}_{R}, \lambda_{R}} g_{\boldsymbol{k}_{R} \lambda_{R}} \hat{b}_{\boldsymbol{k}_{R} \lambda_{R}} \sin \frac{\theta}{2}\right\}+\right| b\right\rangle\langle g|\left\{-\sum_{\boldsymbol{k}_{L}, \lambda_{L}} g_{\boldsymbol{k}_{L} \lambda_{L}} \hat{b}_{\boldsymbol{k}_{L} \lambda_{L}} \sin \frac{\theta}{2}\right. \\
& \left.+\sum_{\boldsymbol{k}_{R}, \lambda_{R}} g_{\boldsymbol{k}_{R} \lambda_{R}} \hat{b}_{\boldsymbol{k}_{R} \lambda_{R}} \cos \frac{\theta}{2}\right\}+|D\rangle\langle a|\left\{\sum_{\boldsymbol{k}_{L}, \lambda_{L}} g_{\boldsymbol{k}_{L} \lambda_{L}} \hat{b}_{\boldsymbol{k}_{L} \lambda_{L}} \sin \frac{\theta}{2}+\sum_{\boldsymbol{k}_{R}, \lambda_{R}} g_{\boldsymbol{k}_{R} \lambda_{R}} \hat{b}_{\boldsymbol{k}_{R} \lambda_{R}} \cos \frac{\theta}{2}\right\} \\
& +|D\rangle\langle b|\left\{\sum_{\boldsymbol{k}_{L}, \lambda_{L}} g_{\boldsymbol{k}_{L} \lambda_{L}} \hat{b}_{\boldsymbol{k}_{L} \lambda_{L}} \cos \frac{\theta}{2}-\sum_{\boldsymbol{k}_{R}, \lambda_{R}} g_{\boldsymbol{k}_{R} \lambda_{R}} \hat{b}_{\boldsymbol{k}_{R} \lambda_{R}} \sin \frac{\theta}{2}\right\}+\text { H.c. }
\end{aligned}
$$

In the weak-coupling limit, the population of the doubly excited state $|D\rangle$ will be small compared to that of the singly excited states $|a\rangle$ and $|b\rangle$. We can thus neglect the last two terms in Eq. (A11) to obtain

$$
\begin{aligned}
\hat{H}_{S B} & =|a\rangle\left\langle g\left|\left\{\sum_{\boldsymbol{k}_{L}, \lambda_{L}} g_{\boldsymbol{k}_{L} \lambda_{L}} \hat{b}_{\boldsymbol{k}_{L} \lambda_{L}} \cos \frac{\theta}{2}+\sum_{\boldsymbol{k}_{R}, \lambda_{R}} g_{\boldsymbol{k}_{R} \lambda_{R}} \hat{b}_{\boldsymbol{k}_{R} \lambda_{R}} \sin \frac{\theta}{2}\right\}+\right| b\right\rangle\langle g|\left\{-\sum_{\boldsymbol{k}_{L}, \lambda_{L}} g_{\boldsymbol{k}_{L} \lambda_{L}} \hat{b}_{\boldsymbol{k}_{L} \lambda_{L}} \sin \frac{\theta}{2}+\sum_{\boldsymbol{k}_{R}, \lambda_{R}} g_{\boldsymbol{k}_{R} \lambda_{R}} \hat{b}_{\boldsymbol{k}_{R} \lambda_{R}} \cos \frac{\theta}{2}\right\}+\mathrm{H} . \mathrm{c} . \\
& =|a\rangle\left\langle g\left|\left\{\sum_{\boldsymbol{k}_{L}, \lambda_{L}} g_{a, \boldsymbol{k}_{L} \lambda_{L}} \hat{b}_{\boldsymbol{k}_{L} \lambda_{L}}+\sum_{\boldsymbol{k}_{R}, \lambda_{R}} g_{a, \boldsymbol{k}_{R} \lambda_{R}} \hat{b}_{\boldsymbol{k}_{R} \lambda_{R}}\right\}+\right| b\right\rangle\langle g|\left\{-\sum_{\boldsymbol{k}_{L}, \lambda_{L}} g_{b, \boldsymbol{k}_{L} \lambda_{L}} \hat{b}_{\boldsymbol{k}_{L} \lambda_{L}}+\sum_{\boldsymbol{k}_{R}, \lambda_{R}} g_{b, \boldsymbol{k}_{R} \lambda_{R}} \hat{b}_{k_{R} \lambda_{R}}\right\}+\mathrm{H.c.}
\end{aligned}
$$

with the new system-bath coupling parameters $g_{i, \boldsymbol{k}_{\alpha} \lambda_{\alpha}}(i=$ $a, b ; \alpha=L, R)$ that describe the coupling between the ground and excited eigenstates of the V-system ( $g \leftrightarrow a$ and $g \leftrightarrow b$ ) due to the left and right baths

$$
\begin{gathered}
g_{a, \boldsymbol{k}_{L} \lambda_{L}}=g_{\boldsymbol{k}_{L} \lambda_{L}} \cos \frac{\theta}{2}, \\
g_{a, \boldsymbol{k}_{R} \lambda_{R}}=g_{\boldsymbol{k}_{R} \lambda_{R}} \sin \frac{\theta}{2}, \\
g_{b, \boldsymbol{k}_{L} \lambda_{L}}=-g_{\boldsymbol{k}_{L} \lambda_{L}} \sin \frac{\theta}{2}, \\
g_{b, \boldsymbol{k}_{R} \lambda_{R}}=g_{\boldsymbol{k}_{R} \lambda_{R}} \cos \frac{\theta}{2} .
\end{gathered}
$$

The above equations show that the system-bath coupling of the two-qubit system weakly driven by the left and right baths (A2) is equivalent to that of the three-level V-system, whose two transitions ( $g \leftrightarrow a$ and $g \leftrightarrow b$ ) are driven simultaneously by the two baths.

We now introduce new bath operators $\hat{B}_{i, \alpha}$, which describe the driving of the V-system transitions $g \leftrightarrow i(i=a, b)$ by the $\alpha$ th bath

$$
\hat{B}_{i, \alpha}=\sum_{\boldsymbol{k}_{\alpha}, \lambda_{\alpha}} g_{i, \boldsymbol{k}_{\alpha} \lambda_{\alpha}} \hat{b}_{\boldsymbol{k}_{\alpha} \lambda_{\alpha}}
$$

where $\alpha=L, R$. The system-bath coupling Hamiltonian can now be rewritten as

$$
\hat{H}_{S B}=\hat{\varphi}_{a}^{+} \cdot\left(\hat{B}_{a, L}+\hat{B}_{a, R}\right)+\hat{\varphi}_{b}^{+} \cdot\left(\hat{B}_{b, L}+\hat{B}_{b, R}\right)+\text { H.c. },
$$

where the V-system jump operators are defined as $\hat{\varphi}_{i}^{+}=$ $|i\rangle\langle g| i=a, b$. Note that Eq. (A18) is identical to Eq. (3) of Sun et al. [39].

To further simplify Eq. (A18) we define the "cumulative" bath operators

$$
\hat{B}_{i}=\sum_{\alpha=L, R} \hat{B}_{i, \alpha}
$$

which induce the transitions $|g\rangle \leftrightarrow|i\rangle(i=a, b)$ between the ground and excited eigenstates of the V-system due to the combined action of both the left and right baths. In terms of the cumulative bath operators, the system-bath interaction Hamiltonian can be expressed in a compact form

$$
\hat{H}_{S B}=\hat{\varphi}_{a}^{+} \cdot \hat{B}_{a}+\hat{\varphi}_{b}^{+} \cdot \hat{B}_{b}+\text { H.c. }=\sum_{i=a, b}\left\{\hat{\varphi}_{i}^{+} \cdot \hat{B}_{i}+\hat{\varphi}_{i}^{-} \cdot \hat{B}_{i}^{\dagger}\right\},
$$

where $\hat{\varphi}_{i}^{-}=\left(\hat{\varphi}_{i}^{+}\right)^{\dagger}$. This expression will serve as a starting point for deriving the BR master equations in Appendix A 2.

\section{System-bath interaction Hamiltonian for two qubits interacting with incoherent radiation fields}

Thus far, our treatment of the system-bath coupling has been completely general. In this section, we specialize this treatment to the case of light-matter coupling in the electric dipole approximation as necessary to describe quantum systems driven by incoherent light [16,29]. At this point, the left (hot) bath will be identified with $x$-polarized incoherent 
light at $T_{L}=5800 \mathrm{~K}$ pumping the left qubit, whereas the right (cold) bath will represent isotropic incoherent electromagnetic vacuum modes ( $\left.T_{R}=0 \mathrm{~K}\right)$ coupled to the right qubit, causing spontaneous emission (see Fig. 1 of the main text). The interaction Hamiltonian of the two-qubit system driven by the incoherent radiation baths defined above is

$$
\hat{H}_{S B}^{\text {dip }}=-\boldsymbol{\mu}_{L} \cdot \boldsymbol{E}_{L}-\boldsymbol{\mu}_{R} \cdot \boldsymbol{E}_{R},
$$

where

$$
\boldsymbol{E}_{\alpha}=i \sum_{\boldsymbol{k}_{\alpha} \lambda_{\alpha}} \sqrt{\frac{\omega_{\boldsymbol{k}_{\alpha}}}{2 \epsilon_{0} V_{\alpha}}} \boldsymbol{\varepsilon}_{\boldsymbol{k}_{\alpha} \lambda_{\alpha}} \hat{b}_{\boldsymbol{k}_{\alpha} \lambda_{\alpha}}+\text { H.c. } \quad(\alpha=L, R)
$$

is the electric field vector of the $\alpha$ th radiation bath with the polarization vector $\boldsymbol{\varepsilon}_{\boldsymbol{k}_{\alpha} \lambda_{\alpha}}$ and frequency $\omega_{\boldsymbol{k}_{\alpha}}$, and $V_{\alpha}$ is the photon volume.

Note that because the transition dipole moment operators $\boldsymbol{\mu}_{\alpha}$ in Eq. (A21) have only off-diagonal matrix elements in the individual qubit (or site) basis, i.e., $\boldsymbol{\mu}_{L}=\boldsymbol{\mu}_{e_{L} g_{L}}\left|e_{L}\right\rangle\left\langle g_{L}\right|=$ $\boldsymbol{\mu}_{e_{L} g_{L}} \sigma_{L}^{+}$and $\boldsymbol{\mu}_{R}=\boldsymbol{\mu}_{e_{R} g_{R}}\left|e_{R}\right\rangle\left\langle g_{R}\right|=\boldsymbol{\mu}_{e_{R} g_{R}} \sigma_{R}^{+}$, we can rewrite Eq. (A21) in a form identical to Eq. (A2)

$$
\hat{H}_{S B}^{\text {dip }}=\sum_{\boldsymbol{k}_{L}, \lambda_{L}} g_{\boldsymbol{k}_{L} \lambda_{L}} \hat{\sigma}_{L}^{+} \hat{b}_{\boldsymbol{k}_{L} \lambda_{L}}+\sum_{\boldsymbol{k}_{R}, \lambda_{R}} g_{\boldsymbol{k}_{R} \lambda_{R}} \hat{\sigma}_{R}^{+} \hat{b}_{\boldsymbol{k}_{R} \lambda_{R}}+\text { H.c. }
$$

with the system-bath coupling coefficients identified as

$$
g_{\boldsymbol{k}_{\alpha} \lambda_{\alpha}}=-i \sqrt{\frac{\omega_{k_{\alpha}}}{2 \epsilon_{0} V_{\alpha}}}\left\{\boldsymbol{\mu}_{e_{\alpha} g_{\alpha}} \cdot \boldsymbol{\varepsilon}_{\boldsymbol{k}_{\alpha} \lambda_{\alpha}}\right\}
$$

where $\boldsymbol{\mu}_{e_{\alpha} g_{\alpha}}$ is the transition dipole moment vector for the $\left|g_{\alpha}\right\rangle \leftrightarrow\left|e_{\alpha}\right\rangle$ transition in the $\alpha$-th qubit.

Using the results derived in the previous section, the matter-field coupling Hamiltonian (A21) takes the form

$$
\begin{aligned}
\hat{H}_{S B}^{\mathrm{dip}} & =|a\rangle\left\langle g\left|\left\{\sum_{\boldsymbol{k}_{L}, \lambda_{L}} g_{a, \boldsymbol{k}_{L} \lambda_{L}} \hat{b}_{\boldsymbol{k}_{L} \lambda_{L}}+\sum_{\boldsymbol{k}_{R}, \lambda_{R}} g_{a, \boldsymbol{k}_{R} \lambda_{R}} \hat{b}_{\boldsymbol{k}_{R} \lambda_{R}}\right\}+\right| b\right\rangle\langle g|\left\{-\sum_{\boldsymbol{k}_{L}, \lambda_{L}} g_{b, \boldsymbol{k}_{L} \lambda_{L}} \hat{b}_{\boldsymbol{k}_{L} \lambda_{L}}+\sum_{\boldsymbol{k}_{R}, \lambda_{R}} g_{b, \boldsymbol{k}_{R} \lambda_{R}} \hat{b}_{k_{R} \lambda_{R}}\right\}+\mathrm{H} . \mathrm{c} . \\
& =\sum_{i=a, b}\left\{\hat{\varphi}_{i}^{+} \cdot \hat{B}_{i}+\hat{\varphi}_{i}^{-} \cdot \hat{B}^{\dagger}\right\},
\end{aligned}
$$

where we will henceforth assume that the cumulative bath operators [Eqs. (A19) and (A17)] are defined with the system-bath coupling coefficients given by Eq. (A24).

The light-matter coupling coefficients $g_{i, \boldsymbol{k}_{\alpha} \lambda_{\alpha}}$ that parametrize the system-radiation field interaction Hamiltonian (A25) in the energy eigenstate basis can be expressed as

$$
g_{i, \boldsymbol{k}_{\alpha} \lambda_{\alpha}}=-i \sqrt{\frac{\omega_{k_{L}}}{2 \epsilon_{0} V_{L}}}\left\{\boldsymbol{\mu}_{i g} \cdot \boldsymbol{\varepsilon}_{\boldsymbol{k}_{\alpha} \lambda_{\alpha}}\right\}
$$

where $\boldsymbol{\mu}_{i g}$ are the matrix elements of the transition dipole moment of the V-system in the energy eigenstate basis

$$
\boldsymbol{\mu}=\sum_{i=a, b} \boldsymbol{\mu}_{i g}|i\rangle\langle g|
$$

To see this, we will show that Eq. (A26) is equivalent to Eqs. (A13)-(A16) under the assumption that the total transition dipole moment of the two-qubit system is equal to the sum of the individual qubit's transition dipoles

$$
\boldsymbol{\mu}=\boldsymbol{\mu}_{e_{L} g_{L}}\left|e_{L}\right\rangle\left\langle g_{L}\left|+\boldsymbol{\mu}_{e_{R} g_{R}}\right| e_{R}\right\rangle\left\langle g_{R}\right|+\text { H.c. }
$$

Using Eqs. (A9) and (A10) in Eq. (A28) and neglecting the transitions to and from the doubly excited state $|D\rangle$, we find

$$
\begin{aligned}
\boldsymbol{\mu} & =\boldsymbol{\mu}_{e_{L} g_{L}}\left\{\cos \frac{\theta}{2}|a\rangle\left\langle g\left|-\sin \frac{\theta}{2}\right| b\right\rangle\langle g|\right\}+\boldsymbol{\mu}_{e_{R} g_{R}}\left\{\sin \frac{\theta}{2}|a\rangle\left\langle g\left|+\cos \frac{\theta}{2}\right| b\right\rangle\langle g|\right\}+\text { H.c. } \\
& =\left\{\boldsymbol{\mu}_{e_{L} g_{L}} \cos \frac{\theta}{2}+\boldsymbol{\mu}_{e_{R} g_{R}} \sin \frac{\theta}{2}\right\}|a\rangle\left\langle g\left|+\left\{-\boldsymbol{\mu}_{e_{L} g_{L}} \sin \frac{\theta}{2}+\boldsymbol{\mu}_{e_{R} g_{R}} \cos \frac{\theta}{2}\right\}\right| b\right\rangle\langle g|+\text { H.c. }
\end{aligned}
$$

Comparing this result with Eq. (A27), we find the transition dipole matrix elements between the ground and excited states of the V-system as

$$
\begin{gathered}
\boldsymbol{\mu}_{a g}=\left\{\boldsymbol{\mu}_{e_{L} g_{L}} \cos \frac{\theta}{2}+\boldsymbol{\mu}_{e_{R} g_{R}} \sin \frac{\theta}{2}\right\}, \\
\boldsymbol{\mu}_{b g}=\left\{-\boldsymbol{\mu}_{e_{L} g_{L}} \sin \frac{\theta}{2}+\boldsymbol{\mu}_{e_{R} g_{R}} \cos \frac{\theta}{2}\right\} .
\end{gathered}
$$

Solving for $\mu_{e_{L} g_{L}}$ and $\mu_{e_{R} g_{R}}$, we obtain

$$
\begin{aligned}
& \boldsymbol{\mu}_{e_{L} g_{L}}=\left\{\boldsymbol{\mu}_{a g} \cos \frac{\theta}{2}-\boldsymbol{\mu}_{b g} \sin \frac{\theta}{2}\right\}, \\
& \boldsymbol{\mu}_{e_{R} g_{R}}=\left\{\boldsymbol{\mu}_{a g} \sin \frac{\theta}{2}+\boldsymbol{\mu}_{b g} \cos \frac{\theta}{2}\right\} .
\end{aligned}
$$


The light-matter coupling coefficients in the energy eigenbasis are therefore related to those in the site basis as

$$
\begin{gathered}
g_{\boldsymbol{k}_{L} \lambda_{L}}=-i \sqrt{\frac{\omega_{k_{L}}}{2 \epsilon_{0} V_{L}}}\left\{\boldsymbol{\mu}_{e_{L} g_{L}} \cdot \boldsymbol{\varepsilon}_{\boldsymbol{k}_{L} \lambda_{L}}\right\}=-i \sqrt{\frac{\omega_{k_{L}}}{2 \epsilon_{0} V_{L}}}\left\{\boldsymbol{\mu}_{a g} \cos \frac{\theta}{2}-\boldsymbol{\mu}_{b g} \sin \frac{\theta}{2}\right\} \cdot \boldsymbol{\varepsilon}_{\boldsymbol{k}_{L} \lambda_{L}} \\
=-i \sqrt{\frac{\omega_{k_{L}}}{2 \epsilon_{0} V_{L}}}\left\{\boldsymbol{\mu}_{a g} \cdot \boldsymbol{\varepsilon}_{\boldsymbol{k}_{L} \lambda_{L}}\right\} \cos \frac{\theta}{2}+i \sqrt{\frac{\omega_{k_{L}}}{2 \epsilon_{0} V_{L}}}\left\{\boldsymbol{\mu}_{b g} \cdot \boldsymbol{\varepsilon}_{\boldsymbol{k}_{L} \lambda_{L}}\right\} \sin \frac{\theta}{2}=g_{a, \boldsymbol{k}_{L} \lambda_{L}} \cos \frac{\theta}{2}-g_{b, \boldsymbol{k}_{L} \lambda_{L}} \sin \frac{\theta}{2}, \\
g_{\boldsymbol{k}_{R} \lambda_{R}}=-i \sqrt{\frac{\omega_{k_{R}}}{2 \epsilon_{0} V_{R}}}\left\{\boldsymbol{\mu}_{e_{R} g_{R}} \cdot \boldsymbol{\varepsilon}_{\boldsymbol{k}_{\boldsymbol{R}} \lambda_{R}}\right\}=-i \sqrt{\frac{\omega_{k_{R}}}{2 \epsilon_{0} V_{R}}}\left\{\boldsymbol{\mu}_{a g} \sin \frac{\theta}{2}+\boldsymbol{\mu}_{b g} \cos \frac{\theta}{2}\right\} \cdot \boldsymbol{\varepsilon}_{\boldsymbol{k}_{\boldsymbol{R}} \lambda_{R}} \\
=-i \sqrt{\frac{\omega_{k_{R}}}{2 \epsilon_{0} V_{R}}}\left\{\boldsymbol{\mu}_{a g} \cdot \boldsymbol{\varepsilon}_{\boldsymbol{k}_{R} \lambda_{R}}\right\} \sin \frac{\theta}{2}-i \sqrt{\frac{\omega_{k_{R}}}{2 \epsilon_{0} V_{R}}}\left\{\boldsymbol{\mu}_{b g} \cdot \boldsymbol{\varepsilon}_{\boldsymbol{k}_{\boldsymbol{R}} \lambda_{R}}\right\} \cos \frac{\theta}{2} \\
=g_{a, \boldsymbol{k}_{R} \lambda_{R}} \sin \frac{\theta}{2}+g_{b, \boldsymbol{k}_{R} \lambda_{R}} \cos \frac{\theta}{2} .
\end{gathered}
$$

Equations (A34) and (A35) are identical to those obtained by inverting Eqs. (A13)-(A16). They establish a connection between the light-matter coupling coefficients in the site basis and those in the energy eigenstate basis.

\section{Quantum master equations in the energy eigenbasis}

In the previous sections, we have shown that the two-qubit system coupled to two independent thermal baths is equivalent to a three-level $\mathrm{V}$-system, whose $g \leftrightarrow i$ transitions $(i=a, b)$ are coupled simultaneously to the left and right baths [see Eq. (A25)]. In this Appendix, we derive the BR quantum master equations for the $\mathrm{V}$-system in the energy eigenbasis, and show that they can be mapped to those of the V-system driven by $x$-polarized incoherent radiation [Eq. (1) of the main text]. We also establish the relationship between the various parameters of these BR equations.

We begin by expressing the system, bath and systembath interaction Hamiltonians in the energy eigenbasis. The V-system Hamiltonian is obtained by diagonalizing the twoqubit Hamiltonian (A1) and neglecting the doubly excited state as described above

$$
\hat{H}_{S}=\sum_{i=g, a, b} E_{i}\left|E_{i}\right\rangle\left\langle E_{i}\right|,
$$

where the two quasidegenerate excited states are denoted by $\left|E_{i}\right\rangle=|i\rangle, \quad i=a, b$ and $\left|E_{g}\right\rangle=|g\rangle$ is the ground state.

The Hamiltonians of the thermal baths representing incoherent radiation fields are given by

$$
\hat{H}_{B}=\hat{H}_{B}^{L}+\hat{H}_{B}^{R}=\sum_{\boldsymbol{k}_{L}, \lambda_{L}} \omega_{k_{L}} \hat{b}_{\boldsymbol{k}_{L} \lambda_{L}}^{\dagger} \hat{b}_{\boldsymbol{k}_{L} \lambda_{L}}+\sum_{\boldsymbol{k}_{R}, \lambda_{R}} \omega_{k_{R}} \hat{b}_{\boldsymbol{k}_{R} \lambda_{R}}^{\dagger} \hat{b}_{\boldsymbol{k}_{R} \lambda_{R}},
$$

where $\hat{H}_{B}^{\alpha}=\sum_{\boldsymbol{k}_{\alpha}, \lambda_{\alpha}} \omega_{k_{\alpha}} \hat{b}_{\boldsymbol{k}_{\alpha} \lambda_{\alpha}}^{\dagger} \hat{b}_{\boldsymbol{k}_{\alpha} \lambda_{\alpha}} ; \alpha=L, R$, and the operators $\hat{b}_{\boldsymbol{k}_{L} \lambda_{L}}^{\dagger} \hat{b}_{\boldsymbol{k}_{L} \lambda_{L}}$ create and annihilate incoherent photons with wave vectors $\boldsymbol{k}_{\alpha}$ and polarizations $\lambda_{\alpha}$.

We have shown in the previous Appendix that the Hamiltonian of the two-qubit system interacting with two thermal baths in the site basis (A21)-(A23) can be transformed to in the eigenstate basis of the related V-system (A25). The system-bath interaction (A20) in the interaction picture can be written as

$\hat{H}_{S B}(t)=\sum_{i}\left\{\hat{\varphi}_{i}^{+}(t) \cdot \hat{B}_{i}(t)+\hat{\varphi}_{i}^{-}(t) \cdot \hat{B}_{i}^{\dagger}(t)\right\}=\hat{V}^{+}(t)+\hat{V}^{-}(t)$,

where the cumulative bath operators $\hat{B}_{i}$ are defined by Eqs. (A19) and (A17) with the system-bath (light-matter) coupling coefficients given by Eq. (A26).

We now proceed to derive the BR master equations for the reduced density operator $\rho$ of the $\mathrm{V}$-system coupled to two independent radiation baths in the interaction picture. The Liouville-von Neumann equation for the reduced density operator is [41]

$$
\dot{\rho}=-\int_{0}^{\infty} d t^{\prime} \operatorname{Tr}_{B}\left[\hat{H}_{S B}(t),\left[\hat{H}_{S B}\left(t-t^{\prime}\right), \rho(t) \otimes \rho_{B}\right]\right],
$$

where $\operatorname{Tr}_{B}$ denotes the trace over the bath modes. Substituting Eq. (A38) into Eq. (A39), the master equation in the BornMarkov approximation can be written as

$$
\begin{aligned}
\dot{\rho}=- & \int_{0}^{\infty} d t^{\prime} \operatorname{Tr}_{B}\left\{\left[\hat{V}^{+}(t),\left[\hat{V}^{-}\left(t-t^{\prime}\right), \rho(t) \otimes \rho_{B}\right]\right]+\left[\hat{V}^{-}(t),\left[\hat{V}^{+}\left(t-t^{\prime}\right), \rho(t) \otimes \rho_{B}\right]\right]\right\} \\
=- & \int_{0}^{\infty} d t^{\prime} \sum_{i, j=a}^{b}\left\{e^{i\left(\varepsilon_{i}-\varepsilon_{j}\right) t}\left(\hat{\varphi}_{i}^{+} \hat{\varphi}_{j}^{-} \rho\right) e^{i \varepsilon_{j} t^{\prime}} \operatorname{Tr}_{B}\left(\hat{B}_{i}(t) \hat{B}_{j}^{\dagger}\left(t-t^{\prime}\right) \rho_{B}\right)-e^{i\left(\varepsilon_{i}-\varepsilon_{j}\right) t}\left(\hat{\varphi}_{i}^{+} \rho \hat{\varphi}_{j}^{-}\right) e^{i \varepsilon_{j} t^{\prime}}\right. \\
& \times \operatorname{Tr}_{B}\left(\hat{B}_{i}(t) \rho_{B} \hat{B}_{j}^{\dagger}\left(t-t^{\prime}\right)\right)-e^{i\left(\varepsilon_{i}-\varepsilon_{j}\right) t}\left(\hat{\varphi}_{j}^{-} \rho \hat{\varphi}_{i}^{+}\right) e^{i \varepsilon_{j} t^{\prime}} \operatorname{Tr}_{B}\left(\hat{B}_{j}^{\dagger}\left(t-t^{\prime}\right) \rho_{B} \hat{B}_{i}(t)\right) \\
& +e^{i\left(\varepsilon_{i}-\varepsilon_{j}\right) t}\left(\rho \hat{\varphi}_{j}^{-} \hat{\varphi}_{i}^{+}\right) e^{i \varepsilon_{j} t^{\prime}} \operatorname{Tr}_{B}\left(\rho_{B} \hat{B}_{j}^{\dagger}\left(t-t^{\prime}\right) \hat{B}_{i}(t)\right)+e^{-i\left(\varepsilon_{i}-\varepsilon_{j}\right) t}\left(\hat{\varphi}_{i}^{-} \hat{\varphi}_{j}^{+} \rho\right) e^{-i \varepsilon_{j} t^{\prime}} \\
& \times \operatorname{Tr}_{B}\left(\hat{B}_{i}^{\dagger}(t) \hat{B}_{j}\left(t-t^{\prime}\right) \rho_{B}\right)-e^{-i\left(\varepsilon_{i}-\varepsilon_{j}\right) t}\left(\hat{\varphi}_{i}^{-} \rho \hat{\varphi}_{j}^{+}\right) e^{-i \varepsilon_{j} t^{\prime}} \operatorname{Tr}_{B}\left(\hat{B}_{i}^{\dagger}(t) \rho_{B} \hat{B}_{j}\left(t-t^{\prime}\right)\right) \\
& \left.-e^{-i\left(\varepsilon_{i}-\varepsilon_{j}\right) t}\left(\hat{\varphi}_{j}^{+} \rho \hat{\varphi}_{i}^{-}\right) e^{-i \varepsilon_{j} t^{\prime}} \operatorname{Tr}_{B}\left(\hat{B}_{j}\left(t-t^{\prime}\right) \rho_{B} \hat{B}_{i}^{\dagger}(t)\right)+e^{-i\left(\varepsilon_{i}-\varepsilon_{j}\right) t}\left(\rho \hat{\varphi}_{j}^{+} \hat{\varphi}_{i}^{-}\right) e^{-i \varepsilon_{j} t^{\prime}} \operatorname{Tr}_{B}\left(\rho_{B} \hat{B}_{j}\left(t-t^{\prime}\right) \hat{B}_{i}^{\dagger}(t)\right)\right\}
\end{aligned}
$$




$$
\begin{aligned}
=- & \int_{0}^{\infty} d t^{\prime} \sum_{i, j=a}^{b}\left\{e^{i\left(\varepsilon_{i}-\varepsilon_{j}\right) t}\left(\hat{\varphi}_{i}^{+} \hat{\varphi}_{j}^{-} \rho\right) e^{i \varepsilon_{j} t^{\prime}}\left\langle\hat{B}_{i}(t) \hat{B}_{j}^{\dagger}\left(t-t^{\prime}\right)\right\rangle-e^{i\left(\varepsilon_{i}-\varepsilon_{j}\right) t}\left(\hat{\varphi}_{i}^{+} \rho \hat{\varphi}_{j}^{-}\right) e^{i \varepsilon_{j} t^{\prime}}\left\langle\hat{B}_{j}^{\dagger}\left(t-t^{\prime}\right) \hat{B}_{i}(t)\right\rangle\right. \\
& -e^{i\left(\varepsilon_{i}-\varepsilon_{j}\right) t}\left(\hat{\varphi}_{j}^{-} \rho \hat{\varphi}_{i}^{+}\right) e^{i \varepsilon_{j} t^{\prime}}\left\langle\hat{B}_{i}(t) \hat{B}_{j}^{\dagger}\left(t-t^{\prime}\right)\right\rangle+e^{i\left(\varepsilon_{i}-\varepsilon_{j}\right) t}\left(\rho \hat{\varphi}_{j}^{-} \hat{\varphi}_{i}^{+}\right) e^{i \varepsilon_{j} t^{\prime}}\left\langle\hat{B}_{j}^{\dagger}\left(t-t^{\prime}\right) \hat{B}_{i}(t)\right\rangle \\
& +e^{-i\left(\varepsilon_{i}-\varepsilon_{j}\right) t}\left(\hat{\varphi}_{i}^{-} \hat{\varphi}_{j}^{+} \rho\right) e^{-i \varepsilon_{j} t^{\prime}}\left\langle\hat{B}_{i}^{\dagger}(t) \hat{B}_{j}\left(t-t^{\prime}\right)\right\rangle-e^{-i\left(\varepsilon_{i}-\varepsilon_{j}\right) t}\left(\hat{\varphi}_{i}^{-} \rho \hat{\varphi}_{j}^{+}\right) e^{-i \varepsilon_{j} t^{\prime}}\left\langle\hat{B}_{j}\left(t-t^{\prime}\right) \hat{B}_{i}^{\dagger}(t)\right\rangle \\
& \left.-e^{-i\left(\varepsilon_{i}-\varepsilon_{j}\right) t}\left(\hat{\varphi}_{j}^{+} \rho \hat{\varphi}_{i}^{-}\right) e^{-i \varepsilon_{j} t^{\prime}}\left\langle\hat{B}_{i}^{\dagger}(t) \hat{B}_{j}\left(t-t^{\prime}\right)\right\rangle+e^{-i\left(\varepsilon_{i}-\varepsilon_{j}\right) t}\left(\rho \hat{\varphi}_{j}^{+} \hat{\varphi}_{i}^{-}\right) e^{-i \varepsilon_{j} t^{\prime}}\left\langle\hat{B}_{j}\left(t-t^{\prime}\right) \hat{B}_{i}^{\dagger}(t)\right\rangle\right\} \\
=- & \sum_{i, j=a}^{b}\left\{e^{i\left(\varepsilon_{i}-\varepsilon_{j}\right) t}\left(\hat{\varphi}_{i}^{+} \hat{\varphi}_{j}^{-} \rho\right) \Gamma_{j i}^{-}\left(\varepsilon_{j}\right)-e^{i\left(\varepsilon_{i}-\varepsilon_{j}\right) t}\left(\hat{\varphi}_{i}^{+} \rho \hat{\varphi}_{j}^{-}\right) \Gamma_{j i}^{+}\left(\varepsilon_{j}\right)-e^{i\left(\varepsilon_{i}-\varepsilon_{j}\right) t}\left(\hat{\varphi}_{j}^{-} \rho \hat{\varphi}_{i}^{+}\right) \Gamma_{j i}^{-}\left(\varepsilon_{j}\right)\right. \\
& +e^{i\left(\varepsilon_{i}-\varepsilon_{j}\right) t}\left(\rho \hat{\varphi}_{j}^{-} \hat{\varphi}_{i}^{+}\right) \Gamma_{j i}^{+}\left(\varepsilon_{j}\right)+e^{-i\left(\varepsilon_{i}-\varepsilon_{j}\right) t}\left(\hat{\varphi}_{i}^{-} \hat{\varphi}_{j}^{+} \rho\right) \Gamma_{i j}^{+}\left(\varepsilon_{j}\right)-e^{-i\left(\varepsilon_{i}-\varepsilon_{j}\right) t}\left(\hat{\varphi}_{i}^{-} \rho \hat{\varphi}_{j}^{+}\right) \Gamma_{i j}^{-}\left(\varepsilon_{j}\right) \\
& \left.-e^{-i\left(\varepsilon_{i}-\varepsilon_{j}\right) t}\left(\hat{\varphi}_{j}^{+} \rho \hat{\varphi}_{i}^{-}\right) \Gamma_{i j}^{+}\left(\varepsilon_{j}\right)+e^{-i\left(\varepsilon_{i}-\varepsilon_{j}\right) t}\left(\rho \hat{\varphi}_{j}^{+} \hat{\varphi}_{i}^{-}\right) \Gamma_{i j}^{-}\left(\varepsilon_{j}\right)\right\},
\end{aligned}
$$

where $\varepsilon_{i}=E_{i}-E_{g}$ is the energy difference between the excited state $i=|a\rangle,|b\rangle$ and the ground state $|g\rangle, \rho(t)$, and $\rho_{B}=$ $\rho_{t h}^{L} \otimes \rho_{t h}^{R}$ are the density operators of the system and the baths. Here, we assume that the thermal baths are in thermal equilibrium described by the density operator $\rho_{t h}^{\alpha}=\exp \left(-\beta_{\alpha} \hat{H}_{B}^{\alpha}\right) / Z_{\alpha}$ with the partition function $Z_{\alpha}=\operatorname{Tr}_{B_{\alpha}}\left[\exp \left(-\beta_{\alpha} \hat{H}_{B}^{\alpha}\right)\right]$, where $\beta_{\alpha}=1 / T_{\alpha}$ is the inverse temperature of the $\alpha$ th bath $(\alpha=L, R)$.

Defining the dissipation rates as one-sided Fourier transforms of bath correlation functions

$$
\begin{aligned}
\Gamma_{i j}^{-} & =\int_{0}^{\infty} d t^{\prime} e^{i \varepsilon_{j} t^{\prime}}\left\langle\hat{B}_{j}(t) \hat{B}_{i}^{\dagger}\left(t-t^{\prime}\right)\right\rangle, \\
\Gamma_{i j}^{+} & =\int_{0}^{\infty} d t^{\prime} e^{i \varepsilon_{j} t^{\prime}}\left\langle\hat{B}_{i}^{\dagger}(t) \hat{B}_{j}\left(t-t^{\prime}\right)\right\rangle
\end{aligned}
$$

and transforming Eq. (A40) from the interaction picture back to the Schrödinger picture, we obtain

$$
\begin{aligned}
\dot{\rho}= & i\left[\rho, \hat{H}_{S}\right]-\sum_{i, j=a}^{b}\left\{\left(\hat{\varphi}_{i}^{+} \hat{\varphi}_{j}^{-} \rho\right) \Gamma_{j i}^{-}\left(\varepsilon_{j}\right)-\left(\hat{\varphi}_{i}^{+} \rho \hat{\varphi}_{j}^{-}\right) \Gamma_{j i}^{+}\left(\varepsilon_{j}\right)-\left(\hat{\varphi}_{j}^{-} \rho \hat{\varphi}_{i}^{+}\right) \Gamma_{j i}^{-}\left(\varepsilon_{j}\right)+\left(\rho \hat{\varphi}_{j}^{-} \hat{\varphi}_{i}^{+}\right) \Gamma_{j i}^{+}\left(\varepsilon_{j}\right)\right. \\
& \left.+\left(\hat{\varphi}_{i}^{-} \hat{\varphi}_{j}^{+} \rho\right) \Gamma_{i j}^{+}\left(\varepsilon_{j}\right)-\left(\hat{\varphi}_{i}^{-} \rho \hat{\varphi}_{j}^{+}\right) \Gamma_{i j}^{-}\left(\varepsilon_{j}\right)-\left(\hat{\varphi}_{j}^{+} \rho \hat{\varphi}_{i}^{-}\right) \Gamma_{i j}^{+}\left(\varepsilon_{j}\right)+\left(\rho \hat{\varphi}_{j}^{+} \hat{\varphi}_{i}^{-}\right) \Gamma_{i j}^{-}\left(\varepsilon_{j}\right)\right\} .
\end{aligned}
$$

Having derived the BR equation in operator form (A43), we now project it onto the eigenstates of the V-system to obtain the BR equations for the individual density matrix elements (i.e., populations and coherences in the eigenstate basis). Specifically, we obtain

$$
\begin{aligned}
\dot{\rho}_{a a}= & \langle a|\dot{\rho}| a\rangle=i\left\langle a\left|\left[\rho, \hat{H}_{S}\right]\right| a\right\rangle-\sum_{i, j=a}^{b}\left\{\left\langle a\left|\hat{\varphi}_{i}^{+} \hat{\varphi}_{j}^{-} \rho\right| a\right\rangle \Gamma_{j i}^{-}\left(\varepsilon_{j}\right)-\left\langle a\left|\hat{\varphi}_{i}^{+} \rho \hat{\varphi}_{j}^{-}\right| a\right\rangle \Gamma_{j i}^{+}\left(\varepsilon_{j}\right)\right. \\
& -\left\langle a\left|\hat{\varphi}_{j}^{-} \rho \hat{\varphi}_{i}^{+}\right| a\right\rangle \Gamma_{j i}^{-}\left(\varepsilon_{j}\right)+\left\langle a\left|\rho \hat{\varphi}_{j}^{-} \hat{\varphi}_{i}^{+}\right| a\right\rangle \Gamma_{j i}^{-}\left(\varepsilon_{j}\right)+\left\langle a\left|\hat{\varphi}_{i}^{-} \hat{\varphi}_{j}^{+} \rho\right| a\right\rangle \Gamma_{i j}^{+}\left(\varepsilon_{j}\right) \\
& \left.-\left\langle a\left|\hat{\varphi}_{i}^{-} \rho \hat{\varphi}_{j}^{+}\right| a\right\rangle \Gamma_{i j}^{-}\left(\varepsilon_{j}\right)-\left\langle a\left|\hat{\varphi}_{j}^{+} \rho \hat{\varphi}_{i}^{-}\right| a\right\rangle \Gamma_{i j}^{+}\left(\varepsilon_{j}\right)+\left\langle a\left|\rho \hat{\varphi}_{j}^{+} \hat{\varphi}_{i}^{-}\right| a\right\rangle \Gamma_{i j}^{-}\left(\varepsilon_{j}\right)\right\} .
\end{aligned}
$$

Next, we simplify the remaining nonzero terms in Eq. (A44)

$$
\begin{aligned}
\sum_{i, j=a}^{b}\left\langle a\left|\hat{\varphi}_{i}^{+} \hat{\varphi}_{j}^{-} \rho\right| a\right\rangle \Gamma_{j i}^{-}\left(\varepsilon_{j}\right) & =\sum_{i, j=a}^{b}\langle a \mid i\rangle\langle g \mid g\rangle\langle j|\rho| a\rangle \Gamma_{j i}^{-}\left(\varepsilon_{j}\right)=\sum_{j=a}^{b}\langle j|\rho| a\rangle \Gamma_{j a}^{-}\left(\varepsilon_{j}\right) \\
& =\langle a|\rho| a\rangle \Gamma_{a a}^{-}\left(\varepsilon_{a}\right)+\langle b|\rho| a\rangle \Gamma_{b a}^{-}\left(\varepsilon_{b}\right)=\Gamma_{a a}^{-}\left(\varepsilon_{a}\right) \rho_{a a}+\Gamma_{b a}^{-}\left(\varepsilon_{b}\right) \rho_{b a}, \\
\sum_{i, j=a}^{b}\left\langle a\left|\hat{\varphi}_{i}^{+} \rho \hat{\varphi}_{j}^{-}\right| a\right\rangle \Gamma_{j i}^{+}\left(\varepsilon_{j}\right) & =\sum_{i, j=a}^{b}\langle a \mid i\rangle\langle g|\rho| g\rangle\langle j \mid a\rangle \Gamma_{j i}^{+}\left(\varepsilon_{j}\right)=\Gamma_{a a}^{+}\left(\varepsilon_{a}\right) \rho_{g g}, \\
\sum_{i, j=a}^{b}\left\langle a\left|\hat{\varphi}_{j}^{+} \rho \hat{\varphi}_{i}^{-}\right| a\right\rangle \Gamma_{i j}^{+}\left(\varepsilon_{j}\right) & =\sum_{i, j=a}^{b}\langle a \mid j\rangle\langle g|\rho| g\rangle\langle i \mid a\rangle \Gamma_{i j}^{+}\left(\varepsilon_{j}\right)=\Gamma_{a a}^{+}\left(\varepsilon_{a}\right) \rho_{g g}, \\
\sum_{i, j=a}^{b}\left\langle a\left|\rho \hat{\varphi}_{j}^{+} \hat{\varphi}_{i}^{-}\right| a\right\rangle \Gamma_{i j}^{-}\left(\varepsilon_{j}\right) & =\sum_{i, j=a}^{b}\langle a|\rho| j\rangle\langle g \mid g\rangle\langle i \mid a\rangle \Gamma_{i j}^{-}\left(\varepsilon_{j}\right)=\sum_{j=a}^{b}\langle a|\rho| j\rangle \Gamma_{a j}^{-}\left(\varepsilon_{j}\right) \\
& =\langle a|\rho| a\rangle \Gamma_{a a}^{-}\left(\varepsilon_{a}\right)+\langle a|\rho| b\rangle \Gamma_{a b}^{-}\left(\varepsilon_{b}\right)=\Gamma_{a a}^{-}\left(\varepsilon_{a}\right) \rho_{a a}+\Gamma_{a b}^{-}\left(\varepsilon_{b}\right) \rho_{a b} .
\end{aligned}
$$


Substituting these results into Eq. (A44), we obtain the quantum master equation for the excited-state population

$$
\begin{aligned}
\dot{\rho}_{a a} & =-\left\{\left(\Gamma_{a a}^{-}\left(\varepsilon_{a}\right) \rho_{a a}+\Gamma_{b a}^{-}\left(\varepsilon_{b}\right) \rho_{b a}\right)-\Gamma_{a a}^{+}\left(\varepsilon_{a}\right) \rho_{g g}-\Gamma_{a a}^{+}\left(\varepsilon_{a}\right) \rho_{g g}+\left(\Gamma_{a a}^{-}\left(\varepsilon_{a}\right) \rho_{a a}+\Gamma_{a b}^{-}\left(\varepsilon_{b}\right) \rho_{a b}\right)\right\} \\
& =2 \Gamma_{a a}^{+}\left(\varepsilon_{a}\right) \rho_{g g}-2 \Gamma_{a a}^{-}\left(\varepsilon_{a}\right) \rho_{a a}-\Gamma_{a b}^{-}\left(\varepsilon_{b}\right) \rho_{a b}-\Gamma_{b a}^{-}\left(\varepsilon_{b}\right) \rho_{b a} .
\end{aligned}
$$

Similarly, the quantum master equation for $\rho_{b b}$ is shown to be

$$
\dot{\rho}_{b b}=2 \Gamma_{b b}^{+}\left(\varepsilon_{b}\right) \rho_{g g}-2 \Gamma_{b b}^{-}\left(\varepsilon_{b}\right) \rho_{b b}-\Gamma_{a b}^{-}\left(\varepsilon_{a}\right) \rho_{a b}-\Gamma_{b a}^{-}\left(\varepsilon_{a}\right) \rho_{b a} .
$$

The BR master equation for the coherence term $\rho_{a b}$ becomes

$$
\begin{aligned}
\dot{\rho}_{a b}=\langle a|\dot{\rho}| b\rangle & =i\left\langle a\left|\left[\rho, \hat{H}_{S}\right]\right| b\right\rangle-\sum_{i, j=a}^{b}\left\{\left\langle a\left|\hat{\varphi}_{i}^{+} \hat{\varphi}_{j}^{-} \rho\right| b\right\rangle \Gamma_{j i}^{-}\left(\varepsilon_{j}\right)-\left\langle a\left|\hat{\varphi}_{i}^{+} \rho \hat{\varphi}_{j}^{-}\right| b\right\rangle \Gamma_{j i}^{+}\left(\varepsilon_{j}\right)\right. \\
& -\left\langle a\left|\hat{\varphi}_{j}^{-} \rho \hat{\varphi}_{i}^{+}\right| b\right\rangle \Gamma_{j i}^{-}\left(\varepsilon_{j}\right)+\left\langle a\left|\rho \hat{\varphi}_{j}^{-} \hat{\varphi}_{i}^{+}\right| b\right\rangle \Gamma_{j i}^{-}\left(\varepsilon_{j}\right)+\left\langle a\left|\hat{\varphi}_{i}^{-} \hat{\varphi}_{j}^{+} \rho\right| b\right\rangle \Gamma_{i j}^{+}\left(\varepsilon_{j}\right) \\
& \left.-\left\langle a\left|\hat{\varphi}_{i}^{-} \rho \hat{\varphi}_{j}^{+}\right| b\right\rangle \Gamma_{i j}^{-}\left(\varepsilon_{j}\right)-\left\langle a\left|\hat{\varphi}_{j}^{+} \rho \hat{\varphi}_{i}^{-}\right| b\right\rangle \Gamma_{i j}^{+}\left(\varepsilon_{j}\right)+\left\langle a\left|\rho \hat{\varphi}_{j}^{+} \hat{\varphi}_{i}^{-}\right| b\right\rangle \Gamma_{i j}^{-}\left(\varepsilon_{j}\right)\right\} .
\end{aligned}
$$

The nonzero terms in Eq. (A47) include $\left\langle a\left|\left[\rho, \hat{H}_{S}\right]\right| b\right\rangle=-\Delta \rho_{a b}$ as well as the following terms:

$$
\begin{aligned}
\sum_{i, j=a}^{b}\left\langle a\left|\hat{\varphi}_{i}^{+} \hat{\varphi}_{j}^{-} \rho\right| b\right\rangle \Gamma_{j i}^{-}\left(\varepsilon_{j}\right) & =\sum_{i, j=a}^{b}\langle a \mid i\rangle\langle g \mid g\rangle\langle j|\rho| b\rangle \Gamma_{j i}^{-}\left(\varepsilon_{j}\right)=\sum_{j=a}^{b}\langle j|\rho| b\rangle \Gamma_{j a}^{-}\left(\varepsilon_{j}\right) \\
& =\langle a|\rho| b\rangle \Gamma_{a a}^{-}\left(\varepsilon_{a}\right)+\langle b|\rho| b\rangle \Gamma_{b a}^{-}\left(\varepsilon_{b}\right)=\Gamma_{a a}^{-}\left(\varepsilon_{a}\right) \rho_{a b}+\Gamma_{b a}^{-}\left(\varepsilon_{b}\right) \rho_{b b}, \\
\sum_{i, j=a}^{b}\left\langle a\left|\hat{\varphi}_{i}^{+} \rho \hat{\varphi}_{j}^{-}\right| b\right\rangle \Gamma_{j i}^{+}\left(\varepsilon_{j}\right) & =\sum_{i, j=a}^{b}\langle a \mid i\rangle\langle g|\rho| g\rangle\langle j \mid b\rangle \Gamma_{j i}^{+}\left(\varepsilon_{j}\right)=\Gamma_{b a}^{+}\left(\varepsilon_{b}\right) \rho_{g g}, \\
\sum_{i, j=a}^{b}\left\langle a\left|\hat{\varphi}_{j}^{+} \rho \hat{\varphi}_{i}^{-}\right| b\right\rangle \Gamma_{i j}^{+}\left(\varepsilon_{j}\right) & =\sum_{i, j=a}^{b}\langle a \mid j\rangle\langle g|\rho| g\rangle\langle i \mid b\rangle \Gamma_{i j}^{+}\left(\varepsilon_{j}\right)=\Gamma_{b a}^{+}\left(\varepsilon_{a}\right) \rho_{g g}, \\
\sum_{i, j=a}^{b}\left\langle a\left|\rho \hat{\varphi}_{j}^{+} \hat{\varphi}_{i}^{-}\right| b\right\rangle \Gamma_{i j}^{-}\left(\varepsilon_{j}\right) & =\sum_{i, j=a}^{b}\langle a|\rho| j\rangle\langle g \mid g\rangle\langle i \mid b\rangle \Gamma_{i j}^{-}\left(\varepsilon_{j}\right)=\sum_{j=a}^{b}\langle a|\rho| j\rangle \Gamma_{b j}^{-}\left(\varepsilon_{j}\right) \\
& =\langle a|\rho| a\rangle \Gamma_{b a}^{-}\left(\varepsilon_{a}\right)+\langle a|\rho| b\rangle \Gamma_{b b}^{-}\left(\varepsilon_{b}\right)=\Gamma_{b a}^{-}\left(\varepsilon_{a}\right) \rho_{a a}+\Gamma_{b b}^{-}\left(\varepsilon_{b}\right) \rho_{a b} .
\end{aligned}
$$

We now substitute all the nonzero terms in Eq. (A47) to obtain the BR master equation for $\rho_{a b}$

$$
\dot{\rho}_{a b}=\left[\Gamma_{b a}^{+}\left(\varepsilon_{a}\right) \rho_{g g}-\Gamma_{b a}^{-}\left(\varepsilon_{a}\right) \rho_{a a}\right]+\left[\Gamma_{b a}^{+}\left(\varepsilon_{b}\right) \rho_{g g}-\Gamma_{b a}^{-}\left(\varepsilon_{b}\right) \rho_{b b}\right]-i \Delta \rho_{a b}-\left[\Gamma_{a a}^{-}\left(\varepsilon_{a}\right)+\Gamma_{b b}^{-}\left(\varepsilon_{b}\right)\right] \rho_{a b} .
$$

From Eqs. (A45), (A46), and (A48), the quantum master equations for the V-system in the energy eigenbasis are

$$
\begin{aligned}
& \dot{\rho}_{a a}=2 \Gamma_{a a}^{+}\left(\varepsilon_{a}\right) \rho_{g g}-2 \Gamma_{a a}^{-}\left(\varepsilon_{a}\right) \rho_{a a}-\Gamma_{a b}^{-}\left(\varepsilon_{b}\right) \rho_{a b}-\Gamma_{b a}^{-}\left(\varepsilon_{b}\right) \rho_{b a}, \\
& \dot{\rho}_{b b}=2 \Gamma_{b b}^{+}\left(\varepsilon_{b}\right) \rho_{g g}-2 \Gamma_{b b}^{-}\left(\varepsilon_{b}\right) \rho_{b b}-\Gamma_{a b}^{-}\left(\varepsilon_{a}\right) \rho_{a b}-\Gamma_{b a}^{-}\left(\varepsilon_{a}\right) \rho_{b a}, \\
& \dot{\rho}_{a b}=\left[\Gamma_{b a}^{+}\left(\varepsilon_{a}\right) \rho_{g g}-\Gamma_{b a}^{-}\left(\varepsilon_{a}\right) \rho_{a a}\right]+\left[\Gamma_{b a}^{+}\left(\varepsilon_{b}\right) \rho_{g g}-\Gamma_{b a}^{-}\left(\varepsilon_{b}\right) \rho_{b b}\right]-i \Delta \rho_{a b}-\left[\Gamma_{a a}^{-}\left(\varepsilon_{a}\right)+\Gamma_{b b}^{-}\left(\varepsilon_{b}\right)\right] \rho_{a b},
\end{aligned}
$$

where $\varepsilon_{i}=E_{i}-E_{g}$ is the energy difference between the excited state $i=|a\rangle,|b\rangle$ and the ground state $|g\rangle$, and $\Delta=$ $E_{a}-E_{b}$ is the excited-state splitting. Equations (A49) are identical to Eqs. (18) of the main text, thereby completing our derivation.

\section{Incoherent driving and spontaneous emission rates of the V-system: left and right baths contributions}

Here, we derive the expressions for the incoherent driving and spontaneous decay rates of the $\mathrm{V}$-system [encoded in the dissipation rates $\Gamma_{i j}^{ \pm}$in Eq. (A49)] in terms of the corresponding rates induced by the left and right baths. This completes the derivation of Eq. (19) of the main text and quantifies the left and right baths's contributions to the radiative dynamics of the incoherently driven $\mathrm{V}$-system.

Here, we focus on the experimental realization of the V-system formed by the electric-dipole allowed transitions between the ground and excited states of atomic calcium (see main text and Ref. [29]). Note that the dipole moment vectors of these two transitions are orthogonal. The diagonal rates $\Gamma_{i i}^{ \pm}$ account for incoherent pumping, stimulated, and spontaneous decay of the excited level $|i\rangle, \quad i=a, b$ of the V-system. The off-diagonal rates $\Gamma_{i j}^{+}$are responsible for Fano interference between the transitions $|g\rangle \leftrightarrow|i\rangle$ and $|g\rangle \leftrightarrow|j\rangle$. These quantities will be considered separately below.

To find the dissipation rates $\Gamma_{i j}^{-}$we evaluate the onesided Fourier transform of the bath correlation functions $\left\langle\hat{B}_{i}(0) \hat{B}_{j}^{\dagger}\left(-t^{\prime}\right)\right\rangle$. The evaluation of one-sided Fourier transform 
of the bath correlation function is simplified with the standard integral of the form

$$
\int_{0}^{\infty} d t^{\prime} e^{ \pm i \varepsilon t^{\prime}}=\pi \delta(\varepsilon) \pm i \mathcal{P} \frac{1}{\varepsilon},
$$

where $\mathcal{P}$ is the Cauchy principal value. The real part of the above integral describes the dissipative dynamics of the system, while the imaginary part represents a small bath-induced contribution to the Lamb shift, which will be neglected.

\section{a. Diagonal dissipation rates: Incoherent pumping and spontaneous decay}

Here, we evaluate the diagonal dissipation rates $\Gamma_{i i}^{ \pm}$in Eq. (A49), which incorporate incoherent driving (i.e. incoherent pumping and stimulated emission) as well as spontaneous decay rates of the $i$-th excited state of the V-system $(i=$ $a, b)$. We first evaluate the dissipation rate of the transition $|a\rangle \leftrightarrow|g\rangle$ by taking the Fourier transform (A41) of the correlation function of the cumulative bath operator defined by Eq. (A19). The assumption that the left and right baths are uncorrelated allows us to drop the cross terms of the form $\left\langle\hat{B}_{a, R}(0) \hat{B}_{a, L}^{\dagger}\left(-t^{\prime}\right)\right\rangle$ in the correlation function $\left\langle\hat{B}_{a}(0) \hat{B}_{b}^{\dagger}\left(-t^{\prime}\right)\right\rangle$, and we find

$$
\begin{aligned}
\Gamma_{a a}^{-}= & \int_{0}^{\infty} d t^{\prime} e^{i \varepsilon_{a} t^{\prime}}\left\langle\hat{B}_{a}(0) \hat{B}_{a}^{\dagger}\left(-t^{\prime}\right)\right\rangle \\
= & \int_{0}^{\infty} d t^{\prime} e^{i \varepsilon_{a} t^{\prime}}\left\langle\left[\hat{B}_{a, L}(0)+\hat{B}_{a, R}(0)\right]\left[\hat{B}_{a, L}^{\dagger}\left(-t^{\prime}\right)+\hat{B}_{a, R}^{\dagger}\left(-t^{\prime}\right)\right]\right\rangle \\
= & \int_{0}^{\infty} d t^{\prime} e^{i \varepsilon_{a} t^{\prime}}\left[\left\langle\hat{B}_{a, L}(0) \hat{B}_{a, L}^{\dagger}\left(-t^{\prime}\right)\right\rangle+\left\langle\hat{B}_{a, R}(0) \hat{B}_{a, R}^{\dagger}\left(-t^{\prime}\right)\right\rangle\right] \\
= & \int_{0}^{\infty} d t^{\prime} e^{i \varepsilon_{a} t^{\prime}}\left[\sum_{\boldsymbol{k}_{L}, \lambda_{L}}\left|g_{a, \boldsymbol{k}_{L} \lambda_{L}}\right|^{2}\left(\left\langle\hat{b}_{\boldsymbol{k}_{L} \lambda_{L}}^{\dagger} \hat{b}_{\boldsymbol{k}_{L} \lambda_{L}}\right\rangle+1\right)\right. \\
& \left.+\sum_{\boldsymbol{k}_{R}, \lambda_{R}}\left|g_{a, \boldsymbol{k}_{R} \lambda_{R}}\right|^{2}\left(\left\langle\hat{b}_{\boldsymbol{k}_{R} \lambda_{R}}^{\dagger} \hat{b}_{\boldsymbol{k}_{R} \lambda_{R}}\right\rangle+1\right)\right] \\
= & I_{1}+I_{2}+I_{3}+I_{4} .
\end{aligned}
$$

This equation has four terms that describe incoherent driving and spontaneous emission processes induced by the left and right baths involving the excited eigenstate $|a\rangle$ of the $\mathrm{V}$-system. Below we will consider these terms in turn.

As noted above, the left bath is identified with a beam of $x$-polarized incoherent light directed along the $\hat{z}$ axis, which drives the $\mathrm{V}$-system and has directional dependence. The right bath is maintained at zero temperature and it is isotropic.

The average photon number in the left bath is given by

$$
\bar{n}_{L}^{P o l}=\left\langle\hat{b}_{\boldsymbol{k}_{L} \lambda_{L}}^{\dagger} \hat{b}_{\boldsymbol{k}_{L} \lambda_{L}}\right\rangle=\left\langle n_{\boldsymbol{k}_{L} \lambda_{L}}\right\rangle=\delta_{\hat{\boldsymbol{k}}_{L} \hat{z}} \delta_{\boldsymbol{\varepsilon}_{\boldsymbol{k}_{L} \lambda_{L} \hat{x}}} \bar{n}_{L},
$$

where $\bar{n}_{L}=\left(e^{\omega_{L} / k_{B} T_{L}}-1\right)^{-1}$ is the average photon number of the isotropic blackbody radiation field at $T_{L}=5800 \mathrm{~K}$. The expression (A52) contains only the modes propagating in the direction of the wave vector $\hat{\boldsymbol{k}}_{L}$ and polarized along the $x$ axis (according to the definition of polarized incoherent light). In contrast, isotropic incoherent radiation contains modes with all possible $\hat{\boldsymbol{k}}_{L}$ and $\lambda_{L}$.

The term $I_{1}$ in Eq. (A51) corresponds to incoherent driving of the $g \leftrightarrow a$ transition by the left bath. To calculate this term, we first evaluate the sum over $\boldsymbol{k}_{L}$ and $\lambda_{L}$

$$
\begin{aligned}
& \sum_{\boldsymbol{k}_{L}, \lambda_{L}}\left|g_{a, \boldsymbol{k}_{L} \lambda_{L}}\right|^{2}\left\langle\hat{b}_{\boldsymbol{k}_{L} \lambda_{L}}^{\dagger} \hat{b}_{\boldsymbol{k}_{L} \lambda_{L}}\right\rangle \\
& =\sum_{\boldsymbol{k}_{L} \lambda_{L}}\left|C_{L}\left(\boldsymbol{\mu}_{a g} \cdot \boldsymbol{\varepsilon}_{\boldsymbol{k}_{L} \lambda_{L}}\right)\right|^{2} \delta_{\hat{\boldsymbol{k}}_{L} \hat{z}} \delta_{\boldsymbol{\varepsilon}_{\boldsymbol{k}_{L}} \hat{x}} \bar{n}_{L} \\
& =\sum_{\boldsymbol{k}_{L}, \lambda_{L}}\left|C_{L} \mu_{a g}\left(\hat{\boldsymbol{\mu}}_{a g} \cdot \boldsymbol{\varepsilon}_{\boldsymbol{k}_{L} \lambda_{L}}\right)\right|^{2} \delta_{\hat{\boldsymbol{k}}_{L} \hat{z}} \delta_{\boldsymbol{\varepsilon}_{k_{L} \lambda_{L} \hat{x}} \bar{n}_{L}} \\
& =\sum_{\boldsymbol{k}_{L}}\left|C_{L} \mu_{a g}\left(\hat{\boldsymbol{\mu}}_{a g} \cdot \hat{\boldsymbol{x}}\right)\right|^{2} \bar{n}_{L}=\frac{1}{2} \sum_{\boldsymbol{k}_{L}}\left|g_{a, \boldsymbol{k}_{L}}^{\prime}\right|^{2} \bar{n}_{L} .
\end{aligned}
$$

In Eq. (A53), $\hat{\boldsymbol{\mu}}_{a g}=\boldsymbol{\mu}_{a g} / \mu_{a g}$ is the unit vector in the direction of $\boldsymbol{\mu}_{a g}, g_{a, \boldsymbol{k}_{L}}^{\prime}=C_{L} \mu_{a g}$ is a polarization-independent light-matter coupling coefficient, and $C_{L}=\left[\omega_{\boldsymbol{k}_{L}} / 2 \epsilon_{0} V_{L}\right]^{1 / 2}$. The scalar products $\hat{\boldsymbol{\mu}}_{a g} \cdot \hat{\boldsymbol{x}}$ and $\hat{\boldsymbol{\mu}}_{b g} \cdot \hat{\boldsymbol{x}}$ are both equal to $1 / \sqrt{2}$ [29].

The integral $I_{1}$ is evaluated as shown in Appendix A 4 [see Eq. (A87)]

$$
\begin{aligned}
I_{1} & =\int_{0}^{\infty} d t^{\prime} e^{i \varepsilon_{a} t^{\prime}} \sum_{\boldsymbol{k}_{L}, \lambda_{L}}\left|g_{a, \boldsymbol{k}_{L} \lambda_{L}}\right|^{2}\left\langle\hat{b}_{\boldsymbol{k}_{L} \lambda_{L}}^{\dagger} \hat{b}_{\boldsymbol{k}_{L} \lambda_{L}}\right\rangle \\
& =\frac{1}{2} 2 \pi \sum_{\boldsymbol{k}_{L}, \lambda_{L}}\left|g_{a, \boldsymbol{k}_{L} \lambda_{L}}\right|^{2} \delta\left(\varepsilon_{a}\right) \bar{n}_{L}^{P o l} \\
& =\frac{1}{2}\left(\frac{3}{16 \pi} \gamma_{a a}^{L} \bar{n}_{L}\right)=\frac{1}{2} r_{a a}^{L}
\end{aligned}
$$

with

$$
\gamma_{a a}^{L}=2 \pi \sum_{\boldsymbol{k}_{L}, \lambda_{L}}\left|g_{a, \boldsymbol{k}_{L} \lambda_{L}}\right|^{2} \delta\left(\varepsilon_{a}\right)=\frac{\omega_{a g}^{3}\left|\mu_{a g}\right|^{2}}{3 \pi \epsilon_{0} c^{3}}
$$

being the spontaneous decay rate of the excited state $|a\rangle$ $(a \rightarrow g)$ induced by the left bath. The incoherent pumping rate for the $g \rightarrow a$ transition by the left bath is given by $r_{a a}^{L}=$ $\frac{3}{16 \pi} \gamma_{a a}^{L} \bar{n}_{L}$. The factor of $3 / 16 \pi$ is included because, as pointed out in Appendix A 4, polarized driving rates are attenuated by a factor of $16 \pi / 3$ with respect to those for isotropic driving.

The term $I_{3}$ in Eq. (A51) corresponds to incoherent driving of the $g \leftrightarrow a$ transition by the right bath:

$$
\begin{aligned}
& \int_{0}^{\infty} d t^{\prime} e^{-i \varepsilon_{a} t^{\prime}} \sum_{\boldsymbol{k}_{R}, \lambda_{R}}\left|g_{a, \boldsymbol{k}_{R} \lambda_{R}}\right|^{2}\left\langle\hat{b}_{\boldsymbol{k}_{R} \lambda_{R}}^{\dagger} \hat{b}_{\boldsymbol{k}_{R} \lambda_{R}}\right\rangle \\
& =\sum_{\boldsymbol{k}_{R}, \lambda_{R}}\left|g_{a, \boldsymbol{k}_{R} \lambda_{R}}\right|^{2} \pi \delta\left(\varepsilon_{a}\right) \bar{n}_{R}=\frac{1}{2} \gamma_{a a}^{R} \bar{n}_{R}=0,
\end{aligned}
$$

because the right bath is maintained at zero temperature, and hence does not drive the system $\left(\bar{n}_{R}=0\right)$. Here, $\gamma_{a a}^{R}=$ $2 \pi \sum_{\boldsymbol{k}_{R}, \lambda_{R}}\left|g_{a, \boldsymbol{k}_{R} \lambda_{R}}\right|^{2} \delta\left(\varepsilon_{a}\right)$ is the spontaneous decay rate of state $|a\rangle$ induced by the right bath (see below).

Having calculated the contributions to the dissipation rate $\Gamma_{a a}^{-}$(A51) due to incoherent driving, we now proceed to evaluate the rate of the spontaneous decay transition $a \rightarrow g$ due to the left bath (the term $I_{2}$ in Eq. (A51))

$$
\int_{0}^{\infty} d t^{\prime} e^{i \varepsilon_{a} t^{\prime}} \sum_{\boldsymbol{k}_{L}, \lambda_{L}}\left|g_{a, \boldsymbol{k}_{L} \lambda_{L}}\right|^{2}=\sum_{\boldsymbol{k}_{L}, \lambda_{L}}\left|g_{a, \boldsymbol{k}_{L} \lambda_{L}}\right|^{2} \pi \delta\left(\varepsilon_{a}\right)=\frac{1}{2} \gamma_{a a}^{L} .
$$


Finally, the spontaneous decay rate of state $|a\rangle$ due to the right bath is given by the fourth term in the last line of Eq. (A51)

$$
\int_{0}^{\infty} d t^{\prime} e^{i \varepsilon_{a} t^{\prime}} \sum_{\boldsymbol{k}_{R}, \lambda_{R}}\left|g_{a, \boldsymbol{k}_{R} \lambda_{R}}\right|^{2}=\sum_{\boldsymbol{k}_{R}, \lambda_{R}}\left|g_{a, \boldsymbol{k}_{R} \lambda_{R}}\right|^{2} \pi \delta\left(\varepsilon_{a}\right)=\frac{1}{2} \gamma_{a a}^{R} .
$$

Substituting Eqs. (A54)-(A58) into Eq. (A51), we find the diagonal dissipation rate for the transition $|a\rangle \leftrightarrow|g\rangle$

$$
\Gamma_{a a}^{-}=\frac{1}{2}\left(r_{a a}^{L}+\gamma_{a a}^{L}\right)+\frac{1}{2}\left(r_{a a}^{R}+\gamma_{a a}^{R}\right)=\frac{1}{2}\left(r_{a a}^{L}+\gamma_{a a}^{L}+\gamma_{a a}^{R}\right) .
$$

Proceeding in a similar way, we find the dissipation rate in Eq. (A42) as

$$
\Gamma_{a a}^{+}=\int_{0}^{\infty} d t^{\prime} e^{i \varepsilon_{a} t^{\prime}}\left\langle\hat{B}_{a}^{\dagger}(0) \hat{B}_{a}\left(-t^{\prime}\right)\right\rangle=\frac{1}{2} r_{a a}^{L}+\frac{1}{2} r_{a a}^{R}=\frac{1}{2} r_{a a}^{L} .
$$

Following the same procedure as outlined above, we evaluate the dissipation rates for the transition $|g\rangle \leftrightarrow|b\rangle:$

$$
\begin{gathered}
\Gamma_{b b}^{-}=\frac{1}{2}\left(r_{b b}^{L}+\gamma_{b b}^{L}\right)+\frac{1}{2}\left(r_{b b}^{R}+\gamma_{b b}^{R}\right)=\frac{1}{2}\left(r_{b b}^{L}+\gamma_{b b}^{L}+\gamma_{b b}^{R}\right), \\
\Gamma_{b b}^{+}=\frac{1}{2} r_{b b}^{L}+\frac{1}{2} r_{b b}^{R}=\frac{1}{2} r_{b b}^{L},
\end{gathered}
$$

where $\gamma_{b b}^{\alpha}=2 \pi \sum_{k_{\alpha}, \lambda_{\alpha}}\left|g_{b, k_{\alpha} \lambda_{\alpha}}\right|^{2} \delta\left(\varepsilon_{b}\right)$ is the spontaneous decay rate of the excited state $|b\rangle$ induced by the $\alpha$-th bath, and $r_{b b}^{\alpha}=\bar{n}_{\alpha} \gamma_{b b}^{\alpha}$ are the corresponding incoherent pumping rates.

\section{b. Off-diagonal dissipation rates: Fano interference}

Having calculated the diagonal dissipation rates, which pertain to the individual transitions $|g\rangle \leftrightarrow|i\rangle ; i=a, b$, we now turn to the off-diagonal dissipation rates due to Fano interference between the transitions $|g\rangle \leftrightarrow|a\rangle$ and $|g\rangle \leftrightarrow|b\rangle$. From Eq. (A41), we obtain

$$
\begin{aligned}
\Gamma_{a b}^{-} & =\Gamma_{b a}^{-}=\int_{0}^{\infty} d t^{\prime} e^{i \varepsilon_{a} t^{\prime}}\left\langle\hat{B}_{a}(0) \hat{B}_{b}^{\dagger}\left(-t^{\prime}\right)\right\rangle \\
& =\int_{0}^{\infty} d t^{\prime} e^{i \varepsilon_{a} t^{\prime}}\left\langle\left[\hat{B}_{a, L}(0)+\hat{B}_{a, R}(0)\right]\left[\hat{B}_{b, L}^{\dagger}\left(-t^{\prime}\right)+\hat{B}_{b, R}^{\dagger}\left(-t^{\prime}\right)\right]\right\rangle \\
& =\int_{0}^{\infty} d t^{\prime} e^{i \varepsilon_{a} t^{\prime}}\left[\left\langle\hat{B}_{a, L}(0) \hat{B}_{b, L}^{\dagger}\left(-t^{\prime}\right)\right\rangle+\left\langle\hat{B}_{a, R}(0) \hat{B}_{b, R}^{\dagger}\left(-t^{\prime}\right)\right\rangle\right] \\
& =\int_{0}^{\infty} d t^{\prime} e^{i \varepsilon_{a} t^{\prime}}\left[\sum_{\boldsymbol{k}_{L}, \lambda_{L}} g_{a, \boldsymbol{k}_{L} \lambda_{L}} g_{b, \boldsymbol{k}_{L} \lambda_{L}}^{*}\left\langle\hat{b}_{\boldsymbol{k}_{L} \lambda_{L}} \hat{b}_{\boldsymbol{k}_{L} \lambda_{L}}^{\dagger}\right\rangle+\sum_{\boldsymbol{k}_{R}, \lambda_{R}} g_{a, \boldsymbol{k}_{R} \lambda_{R}} g_{b, \boldsymbol{k}_{R} \lambda_{R}}^{*}\left\langle\hat{b}_{\boldsymbol{k}_{R} \lambda_{R}} \hat{b}_{\boldsymbol{k}_{R \lambda_{R}}^{\dagger}}^{\dagger}\right\rangle\right] \\
& =\int_{0}^{\infty} d t^{\prime} e^{i \varepsilon_{a} t^{\prime}}\left[\sum_{\boldsymbol{k}_{L}, \lambda_{L}} g_{a, \boldsymbol{k}_{L} \lambda_{L}} g_{b, \boldsymbol{k}_{L} \lambda_{L}}^{*}\left(\left\langle\hat{b}_{\boldsymbol{k}_{L} \lambda_{L}}^{\dagger} \hat{b}_{\boldsymbol{k}_{L} \lambda_{L}}\right\rangle+1\right)+\sum_{\boldsymbol{k}_{R}, \lambda_{R}} g_{a, \boldsymbol{k}_{R} \lambda_{R}} g_{b, \boldsymbol{k}_{R} \lambda_{R}}^{*}\left(\left\langle\hat{b}_{\boldsymbol{k}_{R} \lambda_{R}}^{\dagger} \hat{b}_{\boldsymbol{k}_{R} \lambda_{R}}\right\rangle+1\right)\right] \\
& =I_{1}+I_{2}+I_{3}+I_{4} .
\end{aligned}
$$

Here, as before, we have used the assumption that the left and right baths are uncorrelated to drop the interference terms of the kind $\left\langle\hat{B}_{a, L}(0) \hat{B}_{b, R}^{\dagger}\left(-t^{\prime}\right)\right\rangle$, which arise in the correlation function $\left\langle\hat{B}_{a}(0) \hat{B}_{b}^{\dagger}\left(-t^{\prime}\right)\right\rangle$ involving two cumulative bath operators.

The first term in Eq. (A63) describes Fano interference of incoherent driving processes $a \leftrightarrow g$ and $b \leftrightarrow g$ induced by the left bath. Evaluating this term, we obtain

$$
\begin{aligned}
& \sum_{\boldsymbol{k}_{L}, \lambda_{L}} g_{a, \boldsymbol{k}_{L}} g_{b, \boldsymbol{k}_{L}}^{*}\left\langle\hat{b}_{\boldsymbol{k}_{L} \lambda_{L}}^{\dagger} \hat{b}_{\boldsymbol{k}_{L} \lambda_{L}}\right\rangle \\
& =\sum_{\boldsymbol{k}_{L}, \lambda_{L}} C_{L}\left(\boldsymbol{\mu}_{a g} \cdot \boldsymbol{\varepsilon}_{\boldsymbol{k}_{L} \lambda_{L}}\right) C_{L}\left(\boldsymbol{\mu}_{b g} \cdot \boldsymbol{\varepsilon}_{\boldsymbol{k}_{L} \lambda_{L}}\right)\left\langle\hat{b}_{\boldsymbol{k}_{L} \lambda_{L}}^{\dagger} \hat{b}_{\boldsymbol{k}_{L} \lambda_{L}}\right\rangle,
\end{aligned}
$$

where $C_{L}$ is a constant defined below Eq. (A53). Substituting Eq. (A52) into the first term of Eq. (A64) we obtain, following the steps used in deriving Eq. (A53):

$$
\begin{aligned}
& \sum_{\boldsymbol{k}_{L}, \lambda_{L}} g_{a, \boldsymbol{k}_{L} \lambda_{L}} g_{b, \boldsymbol{k}_{L} \lambda_{L}}^{*}\left\langle\hat{b}_{\boldsymbol{k}_{L} \lambda_{L}}^{\dagger} \hat{b}_{\boldsymbol{k}_{L} \lambda_{L}}\right\rangle \\
& =\sum_{\boldsymbol{k}_{L}, \lambda_{L}} C_{L}\left(\boldsymbol{\mu}_{a g} \cdot \boldsymbol{\varepsilon}_{\boldsymbol{k} \lambda}\right) C_{L}\left(\boldsymbol{\mu}_{b g} \cdot \boldsymbol{\varepsilon}_{\boldsymbol{k} \lambda}\right)\left\langle n_{\boldsymbol{k}_{L} \lambda}\right\rangle
\end{aligned}
$$

$$
\begin{aligned}
& =\sum_{\boldsymbol{k}_{L}, \lambda_{L}} C_{L} \mu_{a g} C_{L} \mu_{b g}\left(\hat{\boldsymbol{\mu}}_{a g} \cdot \boldsymbol{\varepsilon}_{\boldsymbol{k}_{L}, \lambda}\right)\left(\hat{\boldsymbol{\mu}}_{b g} \cdot \boldsymbol{\varepsilon}_{\boldsymbol{k}_{L}, \lambda}\right) \delta_{\hat{\boldsymbol{k}}, \hat{z}} \delta_{\boldsymbol{\varepsilon}_{k_{L}, \lambda} \hat{x}} \bar{n}_{L} \\
& =\sum_{\boldsymbol{k}_{L}} C_{L} \mu_{a g} C_{L} \mu_{b g}\left(\hat{\boldsymbol{\mu}}_{a g} \cdot \hat{\boldsymbol{x}}\right)\left(\hat{\boldsymbol{\mu}}_{b g} \cdot \hat{\boldsymbol{x}}\right) \bar{n}_{L} \\
& =\sum_{\boldsymbol{k}_{L}} \frac{1}{2} C_{L} \mu_{a g} C_{L} \mu_{b g} \bar{n}_{L}=\sum_{\boldsymbol{k}_{L}} \frac{1}{2} g_{a, \boldsymbol{k}_{L}}^{\prime} g_{b, \boldsymbol{k}_{L}}^{\prime} \bar{n}_{L},
\end{aligned}
$$

where $g_{i, \boldsymbol{k}_{\alpha}}^{\prime}$ are polarization-independent light-matter coupling coefficients defined below Eq. (A53).

The one-sided Fourier transform of the above term is given by

$$
\begin{aligned}
I_{1} & =\int_{0}^{\infty} d t^{\prime} e^{i \varepsilon_{a} t^{\prime}} \sum_{\boldsymbol{k}_{L}} \frac{1}{2} g_{a, \boldsymbol{k}_{L}}^{\prime} g_{b, \boldsymbol{k}_{L}}^{\prime} \bar{n}_{L} \\
& =\frac{1}{2} \sum_{\boldsymbol{k}_{L}} g_{a, \boldsymbol{k}_{L}}^{\prime} g_{b, \boldsymbol{k}_{L}}^{\prime} \pi \delta\left(\varepsilon_{a}\right) \bar{n}_{L} \\
& =\frac{\pi}{2} \frac{V_{L}}{(2 \pi)^{3}} \int_{0}^{\infty} \frac{\omega_{\boldsymbol{k}_{L}}}{2 \epsilon_{0} V_{L}}\left(\mu_{a g} \mu_{b g}\right) \boldsymbol{k}_{L}^{2} d \boldsymbol{k}_{L} \delta\left(\varepsilon_{a}\right) \bar{n}_{L}
\end{aligned}
$$




$$
\begin{aligned}
& =\frac{\mu_{a g} \mu_{b g}}{32 \pi \epsilon_{0} c^{3}} \int_{0}^{\infty} d \boldsymbol{k}_{L} \omega_{k_{L}}^{3} \delta\left(\omega_{\boldsymbol{k}_{L}}-\omega_{a g}\right) \bar{n}_{L}=\frac{\mu_{a g} \mu_{b g}}{32 \pi \epsilon_{0} c^{3}} \omega_{a g}^{3} \bar{n}_{L} \\
& =\frac{3}{32 \pi} \sqrt{\frac{\omega_{a g}^{3}\left|\mu_{a g}\right|^{2}}{3 \pi \epsilon_{0} c^{3}}} \sqrt{\frac{\omega_{b g}^{3}\left|\mu_{b g}\right|^{2}}{3 \pi \epsilon_{0} c^{3}}} \bar{n}_{L}=\frac{3}{32 \pi} \sqrt{\gamma_{a a}^{L}} \sqrt{\gamma_{b b}^{L}} \bar{n}_{L},
\end{aligned}
$$

where we have used the definitions of spontaneous emission rates induced by the left bath [Eqs. (A55)] and set $\omega_{b g}=\omega_{a g}$, which is an accurate approximation since $|\Delta| \ll\left|\omega_{i g}\right|$.

The linear orientation of the polarized driving by the left bath attenuates the incoherent pumping rate by the factor $16 \pi / 3$ (see Appendix A 4)

$$
I_{1}=\frac{1}{2}\left\{\frac{3}{16 \pi} \sqrt{\gamma_{a a}^{L} \bar{n}_{L}} \sqrt{\gamma_{b b}^{L} \bar{n}_{L}}\right\} .
$$

In terms of the incoherent driving rates due to the left bath defined by Eq. (A53) we find

$$
I_{1}=\frac{1}{2} \sqrt{r_{a a}^{L} r_{b b}^{L}},
$$

where we have defined the incoherent pumping rates due to the left bath $r_{i i}^{L}=\frac{3}{16 \pi} \gamma_{i i}^{L} \bar{n}_{L}$. These rates are the same as defined by Eq. (A54).

The second term in Eq. (A63) describes Fano interference of spontaneous decay processes $a \rightarrow g$ and $b \rightarrow g$ induced by the left bath [see Appendix A 4, Eq. (A84)] [31]:

$$
\begin{aligned}
I_{2} & =\int_{0}^{\infty} d t^{\prime} e^{i \varepsilon_{a} t^{\prime}} \sum_{\boldsymbol{k}_{L}, \lambda_{L}} g_{a, \boldsymbol{k}_{L} \lambda_{L}} g_{b, \boldsymbol{k}_{L} \lambda_{L}}^{*}=\pi \sum_{\boldsymbol{k}_{L} \lambda_{L}} g_{a, \boldsymbol{k}_{L} \lambda_{L}} \boldsymbol{g}_{b, \boldsymbol{k}_{L} \lambda_{L}}^{*} \delta\left(\varepsilon_{a}\right) \\
& =\frac{1}{2} \gamma_{a b}^{L}=\frac{1}{2} p \sqrt{\gamma_{a a}^{L} \gamma_{b b}^{L}}=0,
\end{aligned}
$$

where $p=\hat{\boldsymbol{\mu}}_{a g} \cdot \hat{\boldsymbol{\mu}}_{b g}$ is the transition dipole alignment factor,

As the V-system composed of Ca atoms (see main text) has orthogonal transition dipole moments $\left(\boldsymbol{\mu}_{a g} \perp \boldsymbol{\mu}_{b g}\right)$, there is no interference between the spontaneous decay processes induced by the left bath $\left(I_{2}=0\right)$ [31].

The third term in Eq. (A63) describes Fano interference of incoherent driving processes $a \leftrightarrow g$ and $b \leftrightarrow g$ due to the right bath:

$$
\begin{aligned}
I_{3} & =\int_{0}^{\infty} d t^{\prime} e^{i \varepsilon_{a} t^{\prime}} \sum_{\boldsymbol{k}_{R}, \lambda_{R}} g_{a, \boldsymbol{k}_{R} \lambda_{R}} g_{b, \boldsymbol{k}_{R} \lambda_{R}}^{*}\left\langle\hat{b}_{\boldsymbol{k}_{R} \lambda_{R}}^{\dagger} \hat{b}_{\boldsymbol{k}_{R} \lambda_{R}}\right\rangle \\
& =\int_{0}^{\infty} d t^{\prime} e^{i \varepsilon_{a} t^{\prime}} \sum_{\boldsymbol{k}_{R}, \lambda_{R}} g_{a, \boldsymbol{k}_{R} \lambda_{R} g_{b, \boldsymbol{k}_{R} \lambda_{R}}^{*}} \bar{n}_{R}=0
\end{aligned}
$$

as the right bath does not drive the system $\left(\bar{n}_{R}=0\right)$.

Finally, the fourth term in Eq. (A63) describes Fano interference of spontaneous decay processes $a \rightarrow g$ and $b \rightarrow g$ induced by the right bath. Following the same approach as used above to evaluate the second term, we find [31]:

$$
I_{4}=\int_{0}^{\infty} d t^{\prime} e^{i \varepsilon_{a} t^{\prime}} \sum_{\boldsymbol{k}_{R}, \lambda_{R}} g_{a, \boldsymbol{k}_{R} \lambda_{R}} g_{b, \boldsymbol{k}_{R} \lambda_{R}}^{*}=\frac{1}{2} p \sqrt{\gamma_{a a}^{R} \gamma_{b b}^{R}}=0
$$

since $p=0$ for the $\mathrm{V}$-system of interest here (atomic calcium) as noted above.
Collecting all the terms in Eq. (A63), we find

$$
\Gamma_{a b}^{-}=\Gamma_{b a}^{-}=\frac{1}{2} \sqrt{r_{a a}^{L} r_{b b}^{L}}
$$

and

$$
\Gamma_{a b}^{+}=\frac{1}{2} \sqrt{r_{a a}^{L} r_{b b}^{L}}
$$

Thus the off-diagonal dissipation rates are determined by Fano interference between the incoherent driving processes $g \leftrightarrow a$ and $g \leftrightarrow b$ induced by the left bath.

\section{Incoherent pumping rates: comparison between isotropic and polarized radiation driving of the $\mathrm{V}$-system}

Here, we establish the relation between the pumping rates of the V-system driven by isotropic and polarized radiation baths. To calculate the pumping rates, we first evaluate the spontaneous decay rate of the excited state $|i\rangle$ due to the left bath

$$
\begin{aligned}
\gamma_{i i}^{L} & =2 \pi \sum_{\boldsymbol{k}_{L}, \lambda_{L}}\left|g_{i, \boldsymbol{k}_{L} \lambda_{L}}\right|^{2} \delta\left(\varepsilon_{i}\right) \\
& =2 \pi \sum_{\boldsymbol{k}_{L}, \lambda_{L}}\left|g_{i, \boldsymbol{k}_{L}}\right|^{2}\left(\hat{\boldsymbol{\mu}}_{i g} \cdot \boldsymbol{\varepsilon}_{\boldsymbol{k}_{L} \lambda_{L}}\right)^{2} \delta\left(\varepsilon_{i}\right),
\end{aligned}
$$

where $g_{i, \boldsymbol{k}_{L}}=\sqrt{\frac{\omega_{\boldsymbol{k}_{L}}}{2 \epsilon_{0} V_{L}}} \mu_{i g}$ is the polarization-independent coupling strength defined above.

We begin by evaluating a more general expression for the cross transition rate [32] due to the interference between the transitions $|i\rangle \leftrightarrow|g\rangle$ (of which Eq. (A74) is a particular case corresponding to $i=j$ )

$$
\begin{aligned}
\gamma_{a b}^{L} & =2 \pi \sum_{\boldsymbol{k}_{L}, \lambda_{L}} g_{a, \boldsymbol{k}_{L} \lambda_{L}} g_{b, \boldsymbol{k}_{L} \lambda_{L}}^{*} \delta\left(\varepsilon_{a}\right) \\
& =2 \pi \sum_{\boldsymbol{k}_{L}, \lambda_{L}} g_{a, \boldsymbol{k}_{L}} \boldsymbol{g}_{b, \boldsymbol{k}_{L}}^{*}\left(\hat{\boldsymbol{\mu}}_{a g} \cdot \boldsymbol{\varepsilon}_{\boldsymbol{k}_{L} \lambda_{L}}\right)\left(\hat{\boldsymbol{\mu}}_{b g} \cdot \boldsymbol{\varepsilon}_{\boldsymbol{k}_{L} \lambda_{L}}\right) \delta\left(\varepsilon_{a}\right) .
\end{aligned}
$$

To evaluate Eq. (A75), we take the continuum limit

$$
\sum_{\boldsymbol{k}_{L}} \rightarrow \frac{V_{L}}{(2 \pi)^{3}} \int_{0}^{\infty} \boldsymbol{k}_{L}^{2} d \boldsymbol{k}_{L} \int_{0}^{\pi} \sin \theta d \theta \int_{0}^{2 \pi} d \boldsymbol{\phi},
$$

where $\theta$ is the angle between the wave vector $\boldsymbol{k}_{L}$ and the $z$ axis of the Cartesian coordinates, and $\phi$ is the azimuthal angle. In the spherical polar coordinates, we can write the wave vector as [32]

$$
\boldsymbol{k}_{L}=\left|\boldsymbol{k}_{L}\right|[\sin \theta \cos \phi, \sin \theta \sin \phi, \cos \theta] .
$$

The polarization vectors $\varepsilon_{k_{L} \lambda_{L}} ; \lambda_{L}=1,2$ and the wave vector are orthogonal to each other. This property allows us to express the polarization vectors as

$$
\begin{aligned}
& \boldsymbol{\varepsilon}_{k_{L} 1}=[-\cos \theta \cos \phi,-\cos \theta \sin \phi, \sin \theta], \\
& \boldsymbol{\varepsilon}_{k_{L} 2}=[\sin \phi,-\cos \phi, 0] .
\end{aligned}
$$

The atomic dipole moments of the V-system for the transitions $|i\rangle \leftrightarrow|g\rangle ; \quad i=a, b$ in the $x y$ plane can be represented in the following form:

$$
\begin{aligned}
\boldsymbol{\mu}_{a g} & =\left|\boldsymbol{\mu}_{a g}\right|\left[\cos \phi_{a}, \sin \phi_{a}, 0\right], \\
\boldsymbol{\mu}_{b g} & =\left|\boldsymbol{\mu}_{b g}\right|\left[\cos \phi_{b}, \sin \phi_{b}, 0\right],
\end{aligned}
$$


where $\phi_{i}$ is the angle formed by the dipole moment vector $\boldsymbol{\mu}_{i g}$ and the $x$ axis. Here, we limit our discussion of the dipole moment orientation to the case of $\Delta m=0$ atomic transitions.
However, it is important to note that we obtain the same spontaneous decay rates for the case $\Delta m= \pm 1$.

We now substitute Eqs. (A76)-(A79) into Eq. (A75) to obtain

$$
\begin{aligned}
\gamma_{a b}^{L}= & \frac{V_{L}}{(2 \pi)^{2}} \int_{0}^{\infty} \frac{\omega_{\boldsymbol{k}_{L}}}{2 \epsilon_{0} V_{L}} \mu_{a g} \mu_{b g} \boldsymbol{k}_{L}^{2} d \boldsymbol{k}_{L} \int_{0}^{\pi} \int_{0}^{2 \pi} d \theta d \phi \sin \theta\left[\cos \phi_{a} \cos \phi_{b}\left(\cos ^{2} \theta \cos ^{2} \phi+\sin ^{2} \phi\right)\right. \\
& \left.+\sin \phi_{a} \sin \phi_{b}\left(\cos ^{2} \theta \sin ^{2} \phi+\cos ^{2} \phi\right)-\sin \left(\phi_{a}+\phi_{b}\right) \sin ^{2} \theta \sin \phi \cos \phi\right] \delta\left(\varepsilon_{a}\right) .
\end{aligned}
$$

Evaluating the integrals over the spherical polar angles $\theta$ and $\phi$

$$
\begin{gathered}
\int_{0}^{2 \pi} d \phi \cos ^{2} \phi=\int_{0}^{2 \pi} d \phi \sin ^{2} \phi=\pi ; \int_{0}^{2 \pi} d \phi \sin \phi \cos \phi=0 ; \\
\int_{0}^{\pi} d \theta \quad \sin \theta\left(\cos ^{2} \theta+1\right)=\frac{8}{3},
\end{gathered}
$$

we find the cross transition rate as

$$
\begin{aligned}
\gamma_{a b}^{L} & =\frac{\mu_{a g} \mu_{b g}}{8 \pi^{2} \epsilon_{0}} \int_{0}^{\infty} \omega_{\boldsymbol{k}_{L}} \boldsymbol{k}_{L}^{2} d \boldsymbol{k}_{L} \delta\left(\varepsilon_{a}\right) \frac{8 \pi}{3}\left(\cos \phi_{a} \cos \phi_{b}+\sin \phi_{a} \sin \phi_{b}\right) \\
& =\frac{\mu_{a g} \mu_{b g}}{3 \pi \epsilon_{0} c^{3}} \cos \left(\phi_{a}-\phi_{b}\right) \int_{0}^{\infty} d \omega_{\boldsymbol{k}_{L}} \omega_{\boldsymbol{k}_{L}}^{3} \delta\left(\omega_{\boldsymbol{k}_{L}}-\omega_{a g}\right)=\frac{\mu_{a g} \mu_{b g} \omega_{a g}^{3}}{3 \pi \epsilon_{0} c^{3}} \cos \left(\phi_{a}-\phi_{b}\right) .
\end{aligned}
$$

where $\left(\phi_{a}-\phi_{b}\right)$ is the angle between the dipole moment vectors $\boldsymbol{\mu}_{a g}$ and $\boldsymbol{\mu}_{b g}$ and $p=\cos \left(\phi_{a}-\phi_{b}\right)=\hat{\boldsymbol{\mu}}_{a g} \cdot \hat{\boldsymbol{\mu}}_{b g}$ is the transition dipole alignment factor.

To find the expression for the spontaneous decay rate of the excited state $|i\rangle$ we set $a=b=i$ in Eq. (A82) to obtain

$$
\gamma_{i i}^{L}=2 \pi \sum_{\boldsymbol{k}_{L}, \lambda_{L}}\left|g_{i, \boldsymbol{k}_{L} \lambda_{L}}\right|^{2} \delta\left(\varepsilon_{i}\right)=\frac{\left|\mu_{i g}\right|^{2} \omega_{i g}^{3}}{3 \pi \epsilon_{0} c^{3}} .
$$

Setting $\omega_{a g} \approx \omega_{b g}$ (which is a valid approximation since $|\Delta| \ll\left|\omega_{a g}\right|$ ), we express the cross transition rate (A82) in terms of the spontaneous decay rates of the excited eigenstates $|a\rangle$ and $|b\rangle$

$$
\gamma_{a b}^{L}=\frac{\mu_{a g} \mu_{b g} \omega_{a g}^{3}}{3 \pi \epsilon_{0} c^{3}} \cos \left(\phi_{a}-\phi_{b}\right)=p \sqrt{\frac{\left|\mu_{a g}\right|^{2} \omega_{a g}^{3}}{3 \pi \epsilon_{0} c^{3}}} \sqrt{\frac{\left|\mu_{b g}\right|^{2} \omega_{b g}^{3}}{3 \pi \epsilon_{0} c^{3}}}=p \sqrt{\gamma_{a a}^{L} \gamma_{b b}^{L}} .
$$

The corresponding isotropic incoherent pumping rate is

$$
r_{i i}^{L(I s o)}=\bar{n}_{L} \gamma_{i i}^{L},
$$

where $\bar{n}_{L}=\left(e^{\omega_{k_{L}} / k_{B} T_{L}}-1\right)^{-1}$ is the average photon number of the isotropic radiation field due to the left bath.

The left bath represents a beam of $\hat{x}$-polarized incoherent light which propagates along the $\hat{z}$ direction with the average occupation number

$$
\bar{n}_{L}^{(\mathrm{Pol})}=\delta_{\hat{\boldsymbol{k}}_{L} \hat{z}} \delta_{\boldsymbol{\varepsilon}_{k_{L} \lambda_{L}} \hat{x}} \bar{n}_{L} .
$$

Using this relation, we derive the expression for the (anisotropic) pumping rate due to $x$-polarized incoherent radiation

$$
\begin{aligned}
r_{i i}^{L(\mathrm{Pol})} & =\gamma_{i i}^{L} \bar{n}_{L}^{(\mathrm{Pol})}=2 \pi \sum_{\boldsymbol{k}_{L}, \lambda_{L}}\left|g_{i, \boldsymbol{k}_{L}}\right|^{2}\left(\hat{\boldsymbol{\mu}}_{i g} \cdot \boldsymbol{\varepsilon}_{\boldsymbol{k}_{L} \lambda_{L}}\right)^{2} \delta\left(\varepsilon_{i}\right) \bar{n}_{L}^{(\mathrm{Pol})} \\
& =2 \pi \sum_{\boldsymbol{k}_{L}, \lambda_{L}}\left|g_{i, \boldsymbol{k}_{L}}\right|^{2}\left(\hat{\boldsymbol{\mu}}_{i g} \cdot \boldsymbol{\varepsilon}_{\boldsymbol{k}_{L} \lambda_{L}}\right)^{2} \delta\left(\varepsilon_{i}\right) \delta_{\hat{\boldsymbol{k}}_{L} \hat{z}} \delta_{\boldsymbol{\varepsilon}_{k_{L} \lambda_{L}} \hat{x}} \bar{n}_{L}=2 \pi \sum_{\boldsymbol{k}_{L}}\left|g_{i, \boldsymbol{k}_{L}}\right|^{2}\left(\hat{\boldsymbol{\mu}}_{i g} \cdot \hat{x}\right)^{2} \delta\left(\varepsilon_{i}\right) \delta_{\hat{\boldsymbol{k}}_{L} \hat{z}} \bar{n}_{L} \\
& =2 \pi \frac{V}{(2 \pi)^{3}} \int_{0}^{\infty} \frac{\omega_{\boldsymbol{k}_{L}}}{2 \epsilon_{0} V}\left|\mu_{i g}\right|^{2}\left(\hat{\boldsymbol{\mu}}_{i g} \cdot \hat{x}\right)^{2} \boldsymbol{k}_{L}^{2} d \boldsymbol{k}_{L} \delta\left(\varepsilon_{i}\right) \bar{n}_{L} \\
& =\frac{\left|\mu_{i g}\right|^{2}}{8 \pi^{2} \epsilon_{0}} \int_{0}^{\infty} \omega_{\boldsymbol{k}_{L}}\left(\frac{1}{\sqrt{2}}\right)^{2} \boldsymbol{k}_{L}^{2} d \boldsymbol{k}_{L} \delta\left(\omega_{\boldsymbol{k}_{L}}-\omega_{i g}\right) \bar{n}_{L} \\
& =\frac{\left|\mu_{i g}\right|^{2}}{16 \pi^{2} \epsilon_{0} c^{3}} \int_{0}^{\infty} d \omega_{\boldsymbol{k}_{L}} \omega_{\boldsymbol{k}_{L}}^{3} \delta\left(\omega_{\boldsymbol{k}_{L}}-\omega_{i g}\right) \bar{n}_{L}=\frac{\omega_{i g}^{3}\left|\mu_{i g}\right|^{2}}{16 \pi^{2} \epsilon_{0} c^{3}} \bar{n}_{L}=\frac{3}{16 \pi}\left(\frac{\omega_{i g}^{3}\left|\mu_{i g}\right|^{2}}{3 \pi \epsilon_{0} c^{3}}\right) \bar{n}_{L}=\frac{3}{16 \pi} r_{i i}^{L(I s o)}
\end{aligned}
$$


From Eqs. (A85) and (A87), we see the anisotropic pumping rate is smaller by the factor of $16 \pi / 3$ than the isotropic pumping rate. The polarized incoherent pumping rate due to the left bath can thus be expressed as $r_{i i}^{L}=\frac{3}{16 \pi} r_{i i}^{L(\text { Iso })}$.

\section{Bloch-Redfield master equations for the V-system}

To obtain the BR equations describing the two-qubit system coupled to two incoherent radiation baths (or, equivalently, the $\mathrm{V}$-system with each of the transitions $g \leftrightarrow i$ driven simultaneously by both baths) we substitute the expressions of the dissipation rates $\Gamma_{i j}^{ \pm}$derived above into Eqs. (A49) to obtain

$$
\begin{gathered}
\dot{\rho}_{a a}=r_{a a}^{L} \rho_{g g}-\left(r_{a a}^{L}+\gamma_{a a}^{L}+\gamma_{a a}^{R}\right) \rho_{a a}-\frac{1}{2} \sqrt{r_{a a}^{L} r_{b b}^{L}}\left(\rho_{a b}+\rho_{b a}\right), \\
\dot{\rho}_{b b}=r_{b b}^{L} \rho_{g g}-\left(r_{b b}^{L}+\gamma_{b b}^{L}+\gamma_{b b}^{R}\right) \rho_{b b}-\frac{1}{2} \sqrt{r_{a a}^{L} r_{b b}^{L}}\left(\rho_{a b}+\rho_{b a}\right), \\
\dot{\rho}_{a b}=-i \Delta \rho_{a b}+\sqrt{r_{a a}^{L} r_{b b}^{L}} \rho_{g g}-\frac{1}{2} \sqrt{r_{a a}^{L} r_{b b}^{L}}\left(\rho_{a a}+\rho_{b b}\right) \\
-\frac{1}{2}\left(r_{a a}^{L}+r_{b b}^{L}+\gamma_{a a}^{L}+\gamma_{b b}^{L}+\gamma_{a a}^{R}+\gamma_{b b}^{R}\right) \rho_{a b},
\end{gathered}
$$

where $\gamma_{i i}^{\alpha}$ is the spontaneous decay rate of the excited state $|i\rangle$ induced by the $\alpha$-th bath $(\alpha=L, R, i=a, b)$ given by
Eq. (A74), and $\bar{r}_{i i}^{L}=\bar{n}_{L} \gamma_{i i}^{L}$ is the incoherent pumping rate due to the left bath. Equation (A88) can be recast as the BR quantum master equation describing the $\mathrm{V}$-system driven by polarized incoherent light [Eq. (1) of the main text]

$$
\begin{aligned}
\dot{\rho}_{i i}= & r_{i} \rho_{g g}-\left(r_{i}+\gamma_{i}\right) \rho_{i i}-\frac{1}{2} \sqrt{r_{a} r_{b}}\left(\rho_{a b}+\rho_{b a}\right), \\
\dot{\rho}_{a b}= & \sqrt{r_{a} r_{b}} \rho_{g g}-\frac{1}{2} \sqrt{r_{a} r_{b}}\left(\rho_{a a}+\rho_{b b}\right)-i \Delta \rho_{a b} \\
& -\frac{1}{2}\left(r_{a}+r_{b}+\gamma_{a}+\gamma_{b}\right) \rho_{a b}
\end{aligned}
$$

with $\gamma_{i}=\gamma_{i i}^{L}+\gamma_{i i}^{R}(i=a, b)$ and $r_{i}=r_{i i}^{L}=\bar{n}_{L} \gamma_{i i}^{L}$. We observe that the rates of radiative transitions in the $\mathrm{V}$-system are composed of the contributions due to both the left and right baths. For example, the decay rate of the excited state $|a\rangle$ can be written as $\gamma_{a}=\gamma_{a a}^{L}+\gamma_{a a}^{R}$, as expected for an eigenstate $|a\rangle$ coupled simultaneously to both of the baths via the systemcoupling Hamiltonian (A25). The interference term $\sqrt{r_{a} r_{b}} \rho_{g g}$ is responsible for the generation of Fano coherence (see Appendix A 3 above), which is due to the simultaneous driving of the transitions $g \rightarrow a$ and $g \rightarrow b$ by a single polarization mode of the left radiation bath.
[1] A. Streltsov, G. Adesso, and M. B. Plenio, Colloquium: Quantum coherence as a resource, Rev. Mod. Phys. 89, 041003 (2017).

[2] T. D. Ladd, F. Jelezko, R. Laflamme, Y. Nakamura, C. Monroe, and J. L. O'Brien, Quantum Computers, Nature (London) 464, 45 (2010).

[3] C. L. Degen, F. Reinhard, and P. Cappellaro, Quantum sensing, Rev. Mod. Phys. 89, 035002 (2017).

[4] A. D. Cronin, J. Schmiedmayer, and D. E. Pritchard, Optics and interferometry with atoms and molecules, Rev. Mod. Phys. 81, 1051 (2009).

[5] M. Schlosshauer, Decoherence and The Quantum-to-Classical Transition (Springer-Verlag Berlin Heidelberg, Berlin, 1997).

[6] Y.-C. Cheng and G. R. Fleming, Dynamics of light harvesting in photosynthesis, Annu. Rev. Phys. Chem. 60, 241 (2009).

[7] L. A. Pachón and P. Brumer, Computational methodologies and physical insights into electronic energy transfer in photosynthetic light-harvesting complexes, Phys. Chem. Chem. Phys. 14, 10094 (2012).

[8] P. Brumer, Shedding (Incoherent) light on quantum effects in light-induced biological processes, J. Phys. Chem. Lett. 9, 2946 (2018).

[9] T. V. Tscherbul and P. Brumer, Quantum coherence effects in natural light-induced processes: cis-trans photoisomerization of model retinal under incoherent excitation, Phys. Chem. Chem. Phys. 17, 30904 (2015)

[10] A. Dodin and P. Brumer, Light-induced processes in nature: Coherences in the establishment of the nonequilibrium steady state in model retinal isomerization, J. Chem. Phys. 150, 184304 (2019).

[11] H. G. Hiscock, S. Worster, D. R. Kattnig, C. Steers, Y. Jin, D. E. Manolopoulos, H. Mouritsen, and P. J. Hore, The quantum needle of the avian magnetic compass, Proc. Natl. Acad. Sci. USA 113, 4634 (2016).

[12] P. Brumer and M. Shapiro, Molecular Response in one photon absorption: Coherent pulsed laser vs. thermal incoherent source, Proc. Natl. Acad. Sci. USA 109, 19575 (2012).

[13] T. V. Tscherbul and P. Brumer, Long-Lived Quasistationary Coherences in a $V$-type System Driven by Incoherent Light, Phys. Rev. Lett. 113, 113601 (2014).

[14] I. Kassal, J. Yuen-Zhou, and S. Rahimi-Keshari, Does coherence enhance transport in photosynthesis? J. Phys. Chem. Lett. 4, 362 (2013).

[15] J. Olsina, A. G. Dijkstra, C. Wang, and J. Cao, Can Natural Sunlight Induce Coherent Exciton Dynamics? arXiv:1408.5385.

[16] T. V. Tscherbul and P. Brumer, Partial secular Bloch-Redfield master equation for incoherent excitation of multilevel quantum systems, J. Chem. Phys. 142, 104107 (2015).

[17] A. Dodin, T. V. Tscherbul, and P. Brumer, Quantum dynamics of incoherently driven V-type systems: Analytic solutions beyond the secular approximation, J. Chem. Phys. 144, 244108 (2016).

[18] A. Dodin, T. V. Tscherbul, and P. Brumer, Coherent dynamics of V-type systems driven by time-dependent incoherent radiation, J. Chem. Phys. 145, 244313 (2016).

[19] A. A. Svidzinsky, K. E. Dorfman, and M. O. Scully, Enhancing photovoltaic power by Fano-induced coherence, Phys. Rev. A 84, 053818 (2011).

[20] M. O. Scully, K. R. Chapin, K. E. Dorfman, M. B. Kim, and A. Svidzinsky, Quantum heat engine power can be increased by noise-induced coherence, Proc. Natl. Acad. Sci. USA 108, 15097 (2011).

[21] K. E. Dorfman, D. V. Voronine, S. Mukamel, and M. O. Scully, Photosynthetic reaction center as a quantum heat engine, Proc. Natl. Acad. Sci. USA 110, 2746 (2013). 
[22] G. S. Agarwal, in Quantum Optics, Springer Tracts in Modern Physics No. 70 (Springer, Berlin, 1974), pp. 1-128.

[23] M. Fleischhauer, C. H. Keitel, M. O. Scully, and C. Su, Lasing without inversion and enhancement of the index of refraction via interference of incoherent pump processes, Opt. Commun. 87, 109 (1992).

[24] G. C. Hegerfeldt and M. B. Plenio, Coherence with incoherent light: A new type of quantum beats for a single atom, Phys. Rev. A 47, 2186 (1993).

[25] V. V. Kozlov, Y. Rostovtsev, and M. O. Scully, Inducing quantum coherence via decays and incoherent pumping with application to population trapping, lasing without inversion, and quenching of spontaneous emission, Phys. Rev. A 74, 063829 (2006).

[26] S. Koyu and T. V. Tscherbul, Long-lived quantum coherences in a V-type system strongly driven by a thermal environment, Phys. Rev. A 98, 023811 (2018).

[27] G. S. Agarwal and S. Menon, Quantum interferences and the question of thermodynamic equilibrium, Phys. Rev. A 63, 023818 (2001).

[28] C. Creatore, M. A. Parker, S. Emmott, and A. W. Chin, Efficient Biologically Inspired Photocell Enhanced by Delocalized Quantum States, Phys. Rev. Lett. 111, 253601 (2013).

[29] A. Dodin, T. V. Tscherbul, R. Alicki, A. Vutha, and P. Brumer, Secular versus nonsecular Redfield dynamics and Fano coherences in incoherent excitation: An experimental proposal, Phys. Rev. A 97, 013421 (2018).

[30] L. Mandel and E. Wolf, Optical Coherence and Quantum Optics (Cambridge University Press, Cambridge, UK, 1995), Chap. 13.

[31] A. K. Patnaik and G. S. Agarwal, Cavity-induced coherence effects in spontaneous emissions from preselection of polarization, Phys. Rev. A 59, 3015 (1999).

[32] Z. Ficek and S. Swain, Quantum Interference and Coherence. Theory and Experiments (Springer Verlag, New York, 2005), Chap. 2.1.

[33] T. W. Cronin and J. Marshal, Patterns and properties of polarized light in air and water, Philos. Trans. R. Soc. London B 366, 619 (2011).

[34] S. Johnsen, The Optics of Life: A Biologist's Guide to Light in Nature (Princeton University Press, Princeton, NJ, 2012).

[35] R. J. León-Montiel, I. Kassal, J. P. Torres, Importance of excitation and trapping conditions in photosynthetic environmentassisted energy transport, J. Phys. Chem. B 118, 10588 (2014).

[36] T. V. Tscherbul and P. Brumer, Non-equilibrium stationary coherences in photosynthetic energy transfer under weak-field incoherent illumination, J. Chem. Phys. 148, 124114 (2018).

[37] K. A. Jung and P. Brumer, Energy transfer under natural incoherent light: Effects of asymmetry on efficiency, J. Chem. Phys. 153, 114102 (2020).

[38] P.-Y. Yang and J. Cao, Steady-state analysis of light-harvesting energy transfer driven by incoherent light: From dimers to networks, J. Phys. Chem. Lett. 11, 7204 (2020).

[39] S.-W. Li, C. Y. Cai, and C. P. Sun, Steady quantum coherence in non-equilibrium environment, Ann. Phys. 360, 19 (2015).

[40] H.-P. Breuer and F. Petruccione, The Theory of Open Quantum Systems (Clarendon Press, Oxford, 2006), Chap. 3.4.

[41] K. Blum, Density Matrix Theory and Applications (Springer, 2011), Chap. 8.
[42] F. K. Wilhelm, M. J. Storcz, U. Hartmann, and M. R. Geller, Superconducting Qubits II: Decoherence, in Manipulating Quantum Coherence in Solid State Systems, edited by E. Flatté and I. Tifrea (Springer, Dordrecht, 2007), p. 195.

[43] G. Benenti, G. Casati, K. Saito, and R. S. Whitney, Fundamental aspects of steady-state conversion of heat to work at the nanoscale, Phys. Rep. 694, 1 (2017).

[44] S. Koyu, Noise-induced Coherent Dynamics of Multilevel Quantum Systems Driven by Incoherent Light: A Case Study of the Three-level V-system, Ph.D. thesis, University of Nevada, Reno, 2020.

[45] D. Segal, A. Nitzan, W. B. Davis, M. R. Wasielewski, and M. A. Ratner, Electron transfer rates in bridged molecular systems. II. A steady-state analysis of coherent tunneling and thermal transitions, J. Phys. Chem. B 104, 3817 (2000).

[46] D. Manzano, Quantum transport in networks and photosynthetic complexes at the steady state, PLoS ONE 8, e57041 (2013).

[47] J. Wu, F. Liu, J. Ma, R. J. Silbey, and J. Cao, Efficient energy transfer in light-harvesting systems: Quantum-classical comparison, flux network, and robustness analysis, J. Chem. Phys. 137, 174111 (2012).

[48] R. Kosloff and A. Levy, Quantum heat engines and refrigerators: Continuous devices, Annu. Rev. Phys. Chem. 65, 365 (2014).

[49] P. Rebentrost, M. Mohseni, I. Kassal, S. Lloyd, and A. AspuruGuzik, Environment-assisted quantum transport, New J. Phys. 11, 033003 (2009).

[50] A. W. Chin, A. Datta, F. Caruso, S. F. Huelga, and M. B. Plenio, Noise-assisted energy transfer in quantum networks and lightharvesting complexes, New J. Phys. 12, 065002 (2010).

[51] J. Wu, F. Liu, Y. Shen, J. Cao, and R. J. Silbey, Efficient energy transfer in light-harvesting systems, I: Optimal temperature, reorganization energy and spatial-temporal correlations, New J. Phys. 12, 105012 (2010).

[52] L. Andrews, W. W. Duley, and L. Brewer, Optical spectra of calcium atoms in inert matrices: A new red absorption for $\mathrm{Ca}_{2}$, J. Mol. Spectrosc. 70, 41 (1978).

[53] S. Upadhyay, U. Dargyte, R. P. Prater, V. D. Dergachev, S. A. Varganov, T. V. Tscherbul, D. Patterson, and J. D. Weinstein, Enhanced spin coherence of rubidium atoms in solid parahydrogen, Phys. Rev. B 100, 024106 (2019).

[54] S. Upadhyay, U. Dargyte, V. D. Dergachev, R. P. Prater, S. A. Varganov, T. V. Tscherbul, D. Patterson, and J. D. Weinstein, Spin coherence and optical properties of alkali-metal atoms in solid parahydrogen, Phys. Rev. A 100, 063419 (2019).

[55] J. O. González, L. A. Correa, G. Nocerino, J. P. Palao, D. Alonso and G. Adesso, Testing the validity of the 'Local' and 'Global' GKLS master equations on an exactly solvable model, Open Syst. Inf. Dyn. 24, 1740010 (2017).

[56] P. P. Hofer, M. Perarnau-Llobet, L. D. M. Miranda, G. Haack, R. Silva, J. B. Brask and N. Brunner, Markovian master equations for quantum thermal machines: local versus global approach, New J. Phys. 19, 123037 (2017).

[57] P. P. Potts, Introduction to quantum thermodynamics (Lecture Notes), arXiv:1906.07439v2.

[58] R. Wertheimer, and R. Silbey, On Excitation transfer and relaxation models in low-temperature systems, Chem. Phys. Lett. 75, 243 (1980). 